\title{
Computational modeling of epithelial wound healing: Short and long term chemo-mechanical mechanisms
}

\author{
L. Roldán ${ }^{a, b}$, J.J. Muñoz ${ }^{a, c, d}$, P. Sáez $z^{a, b, d, *}$ \\ ${ }^{a}$ Laboratori de Càlcul Numèric (LaCàN), \\ ${ }^{b}$ E.T.S. de Ingenieros de Caminos, \\ ${ }^{c}$ Department of Mathematics, \\ Universitat Politècnica de Catalunya (UPC), Barcelona, Spain \\ ${ }^{d}$ Barcelona Graduate School of Mathematics (BGSMath), Barcelona, Spain
}

\begin{abstract}
During the lifetime of all living multicellular organisms, wounds in their tissues are frequently observed. The capability of closing those gaps is fundamental for a healthy development. If done deficiently, many diseases may occur from simple inflammation to tumor formation. The wound healing process in epithelial tissue occurs in three different stages. The first one is the assembly of a supra-cellular actomyosin cable and its migration towards the wound edge, triggered by biochemical processes in which calcium plays a distinctive role. How this process is orchestrated following damage remains unclear. Later, after its positioning, the cable contracts driving the tissue towards the gap and reducing the wound area. Finally, cell migration towards the interior of the wound ends up sealing the tissue. In this work, we make use of a mechanical continuum model for the first two stages in order to developed and 2D finite element simulations within a monolithically fully implicit implementation. The model for the actomyosin cable formation involves the coupling of transient calcium ions transport, with actin fibers and myosin motors recruitment and non-linear mechanical response of the tissue. The contraction stage, the active deformation of the previously formed actomyosin cable is taken into account. The relative motion of the myosin motors over the actin filaments is modeled so there exists an active tissue contraction in the direction of those fibers. Upon implementation, the model is capable of performing a wide range of biophysical situations reported experimentally, as we demonstrate in our numerical results. We have been able to rationalize through computational mechanics the firing of calcium in the wound right after damage infliction as well as the consequent formation of actin ring, reproducing nicely what has been reported in biological literature. Thereafter, the numerical model of acto-myosin contraction, fully integrated with the non-linear mechanics of the problem, correlates with the mechanics of wound closure at the actin-ring contraction stage. More importantly, the approach is the first of its kind in the modeling of epithelial and embryonic cell layers, where a wide number of complex mechanics has been integrated and solved though computational methods in engineering. We believe that the simulations will help to unravel new insights in open questions of developmental biology.
\end{abstract}

Keywords: Biological tissue, Wound healing, Constitutive behavior, Finite Elements

\section{Introduction}

Gaps in multicellular tissues and cells occur during the lifetime of an organism as consequence of physiological or pathological processes $[1,2]$. Closure of these gaps is key for the development and functionality of biological organisms. The malfunctioning of the wound closure may lead to diseases such as tumorigenesis

\footnotetext{
*P. Sáez. Laboratori de Càlcul Numèric (LaCàN). E.T.S. de Ingenieros de Caminos, Universitat Politècnica de Catalunya, Jordi Girona 1, 08034 Barcelona, Spain.

Email address: pablo.saez@upc.edu (P. Sáez)
} 
[3]. Clear understanding of this phenomena and the mechanisms involved is of great importance. It is crucial to restore epithelial physiology to protect organisms from infection or to allow a normal development [4]. The complex and orchestrated mechano-biological regulation is coordinated from right after injury through all the healing process and, most important, is observed among different types of tissues.

The process of wound healing develops in three well differentiated time scales, with clear separated mechanisms and goals: 1) actomyosin cable formation (15 minutes approximately), 2) actin ring (or purse ring) contraction, predominant in non adherent substrates, and 3) individual and collective cell migration that ends up closing the gap. The cooperative mechanisms 2 and 3 can last for almost 4 hours. Although some wound area reduction is observed during the early minutes, its magnitude is small compared with the effects of main contraction mechanism in stage 2 [5]. In this paper we will focus on the modelling of stages 1 and 2, as described in Figure 1. For further reference, an excellent review of the different mechanisms in wound healing can be found in [6].

Figure 1: Wound closure evolution in time. Two different steps can be distinguished, a first one where the actomyosin ring is formed and a second one where the contraction of that ring occurs, closing the gap. Adapted from [5].

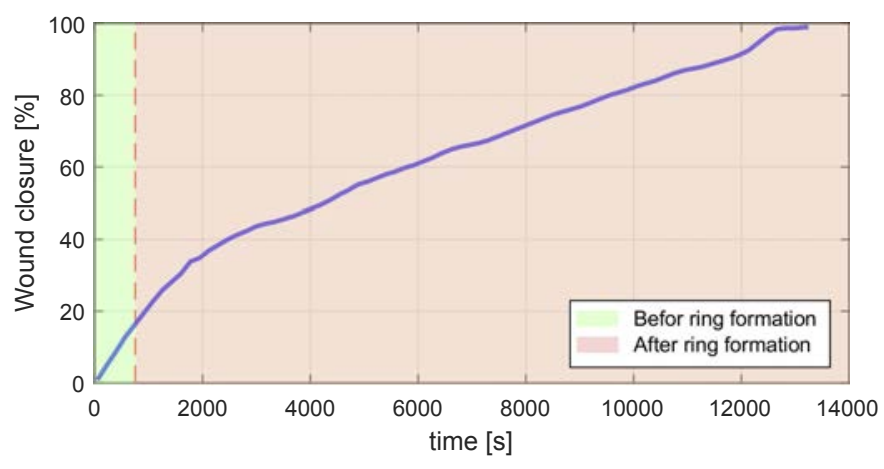

During mechanism 1, calcium plays a fundamental role in wound healing [7] and it has been directly implicated in the formation of the actin ring [8,9,10], mainly through the downstream activation of the Rho family of proteins, which is directly implicated in actin formation. The deformation of the membrane and the diffusion of the calcium concentration promotes a wave of $\mathrm{Ca}^{2+}[8,5]$. Although the exact mechanism of these calcium pike is not clear, it is believed to be dependent on the ion-channels present in the epithelial cells $[8,11]$. Some membrane proteins form pores, or Ion-Channels, that regulate the transport of ions through passive diffusion [12]. The mechanism for the opening or closing of these channels can be controlled by different means, one of them being stress or stretch, in what are called mechanosensitive ion channels $[13,14]$. At the same time, the recruitment of actin fibers and myosin motors, which will be responsible of the active contractibility of the cable seems to be triggered by ion signals. Some studies suggest a correlation between upper and lower bounds for concentration of $\mathrm{Ca}^{2+}$ with the migration of the actomyosin from where is formed towards the wound edge [5].

Mechanism 2 takes place at the cells surrounding the wound. A cable formed by actin filaments, the so called purse string, is formed at the basal epidermis and at the leading edge of the wound, which contracts upon formation $[15,16]$. The purse ring provides the force necessary to draw the wound edges together to achieve re-epithelialisation $[17,16]$ where the key component of this contractile apparatus are the Myosin II motors, assembled in a coordinated manner in the leading edge of the wound margin [18, 16, 19]. Additionally to the actomyosin ring contraction, it has been observed a migration of marginal and submarginal cells (mechanism 3), led by actin based lamellipodial and filopodial protrusions, allowing contact between opposing leading edge cells. Both types of protrusions are key to the binding of epithelial layers, as they allow the tugging of cells among each other [20,16] and closure of large gaps [22]. The cellular protrusion is an individual cell move mechanism while a collective movement mechanism called Kenotaxis (propulsion towards empty spaces) has also been observed in tissues that try to fill empty gaps [23].

\subsection{Actomyosin ring formation}

This mechanism can be summarized as follows and it is illustrated in Fig. 2). The wound produces a 
deformation on the tissue. The deformation is possible because the epithelial tissue is in a so-called pre-stress configuration, which arises mainly due to the action of myosin motors in the micro-scale [22].

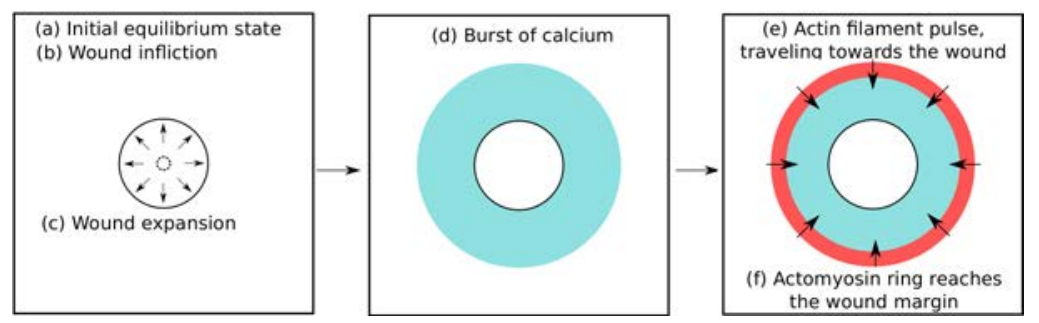

Figure 2: Stages in actomyosin ring forma-
tion. (a) Intact tissue. (b) Wounding. (c)
Expansion. (d) Calcium burst due to me-
chanical deformation. (e) Formation of an
actin ring and motion in the direction of the
wound. (e) An actomyosin cable is formed
as the waves of filaments/motors reach the
wound edge. Adapted from [5].

The cell deformation triggers an increase in the calcium concentration. The increase in calcium concentration arises from the release from both internal and external storage spaces [20, 21, 5]. Once the initial burst of calcium arises, a quick wave of calcium through the tissue is observed [5]. After this initial process, around 40 seconds after ablation, a particular concentration distribution can be observed, with a minimum in the wound edge and a maximum away from it. In Figure 3 it can be noted how a calcium peak migrates towards the wound as time advances. This phenomena can be explained by the opening and closing of the ion channels, ahead and behind of the curve's peak respectably, reacting to the cells deformation. It is also important to remark that, in concordance with Figure 3, the intensity of the calcium profile tends to decrease with time. Note, that no information regarding the calcium distribution during the first $40 \mathrm{~s}$ is available. It is commonly assumed that that calcium generation is larger at locations with larger deformation gradient. In the wounded tissue and at time $t=40 \mathrm{~s}$, this gradient is larger at the wound edge. However, calcium concentration is maximum some 20-25 $\mu \mathrm{m}$ away from the wound front, and no mechanism has been found so far for this distribution.
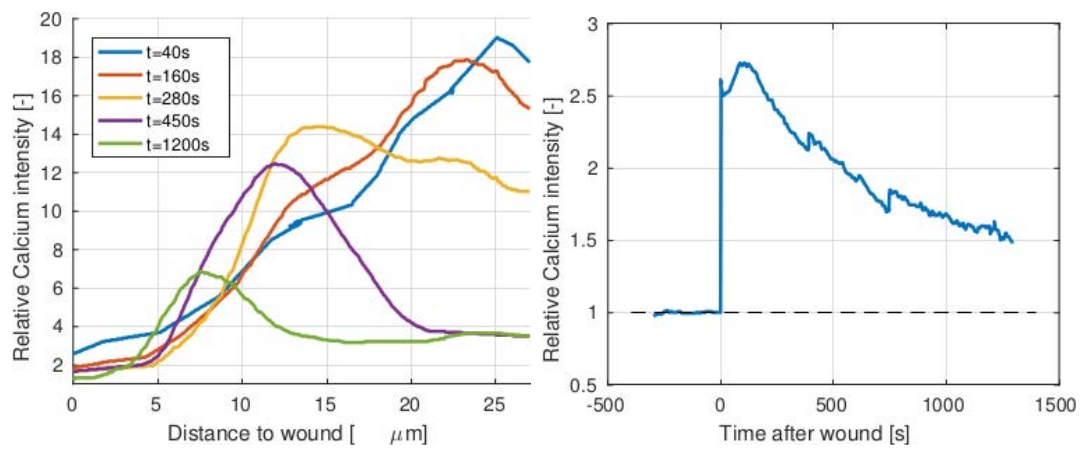

Figure 3: Evolution of calcium relative intensity in time and space. Left: An abrupt jump in calcium intensity is observed when the injury occurs, slowly decaying with the time. Right: spacial calcium relative intensity profiles at different times. A wave of decaying intensity can be observed moving towards the wound margin. Adapted from [5].

One of the most accepted mechanisms for the transport of intracellular calcium ions is the strainactivation of gap junctions at the cell membrane $[28,29]$. At the macro scale, the process can be understood as a diffusion mechanism, as several studies have tried to replicate the transport over different types of tissues. Long et. al. [24] performed a theoretical and experimental study to study the propagation of calcium waves, using a transient diffusion-reaction equation in 1D propagation with a constant diffusion coefficient. Hofer et. al. [25] proposed a similar equation, but with a variable diffusion, which depends on the concentration of calcium. Goldberg et. al. [26] proposed a more complex model for calcium dynamics by coupling the calcium concentration with the cell's average $I P_{3}$ concentration (a secondary messenger molecule used for signal transduction) and the number of open $I P_{3} R$ channels on the membrane, aiming to reproduce a linear and non-linear behavior of the gap junction opening and closure.

Calcium and actin/myosin concentrations correlates, both in space and time, with the calcium peak traveling slightly behind the actomyosin wave [5]. This correspondence can be observed by comparing Figure 3 and Figure 4. Note that the profile for the actin concentration is similar in all the stages, except for the peak that travels towards the wound edge. It seems that high concentrations of calcium prevent the formation of new actin filaments, whereas at intermediate levels the maximum amount of actin is observed 
[5]. This property suggest that there is a zone, with calcium concentration in between a lower and an upper limit, which is beneficial to the recruitment of actin filaments. As the calcium profile evolves, this zone moves, which would explain the migration of the actomyosin peak seen in Figure 4. Calcium concentration regulates the recruitment of actin and myosin, which produces a relative contraction in the cells, affecting calcium distribution, and producing an actomyosin flow that culminates in the actomyosin ring around the wound edge [5]. It was observed that the peak of the intensity profile follows the actin wave, and correlates with the maximum relative individual area reduction of the cells [5]. Although it is clear that calcium waves and acto-myosin concentration correlates in space and time, the results did not provide a reason for the formation of the actin ring at the wound edge (see Figure 4).

Figure 4: Evolution of actin relative intensity profile in time. It can be observed a wave of actin concentration moving towards the wound edge, reaching the vicinity of the gap in the first 20 minutes of the wound healing process. Adapted from [5].

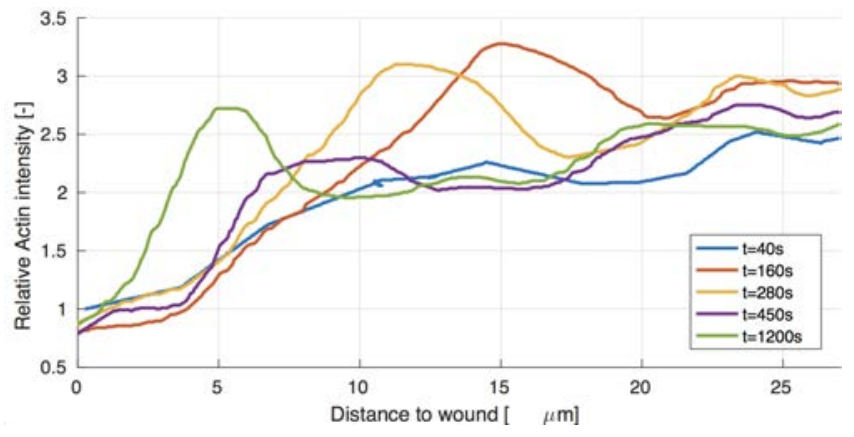

\subsection{Actomyosin ring contraction}

After the actin ring has been formed, the coupling of actin and myosin motors promotes the active contraction of the actin ring. As discussed, the long term closure can be due to the actin ring or a continuous protrusion of the lamillopodia of the cells close to the wound and the crawling of the cells towards the wound center. In this regard, Brugués at. al [22] were able to measure the displacements and stresses in the substrate of an experimental wound closure scenario. In their study they propose a model where the cell crawling at initial stages is the most important mechanism of the wound healing, while in the posterior stages the actomyosin ring contraction gains relevance. In this last stage two aspects dictates the closing efficiency: firstly, the already mentioned ring-contraction mechanism, and secondly, the focal adhesions, which allow the movement of the cells by transmitting part of the force to the substrate [22]. Vedula et. al. [17] found that for diameters above 150um the purse-string mechanism is ineffective. They also reached to the conclusion, that the actomyosin ring contraction appears to be more relevant in cases where there is poor or non-existent extracellular matrix.

To this end, cell in wound regions use mainly two mechanisms to achieve the closure and healing of gaps: 2a) cells close to the wound edge crawl toward the center of the gap using adhesive mechanisms with the substrate [22] and thereafter, 2b) an actin ring formed at the edge of the gap contracts and actively closes the gap (purse-string mechanism) [27, 28]. In situations with a well formed extracellular matrix (ECM), where a stable lamillopodia protrusion are favored, cell crawling seems to be the preferred mechanism to close the wound gap [29, 30]. In small gaps, as those appearing during apoptotic cells [31] and in non-adherent substrates either in-vitro [23] or in embrionic epithelial layers with a non mature ECM [17], the preferred mechanism seems to be the collective formation of the actin-ring and the subsequent contraction $[32,17,19]$. Nevertheless, and after a long debate in the experimental biology field, it seems that both mechanisms work co-operatively at a smaller or higher degree to achieve an efficient and complete wound healing.

This paper focuses on a system with non-adherent substrates and therefore, as described above, in mechanisms dominated by purse-ring-like closure. The exact process through which the cable is contracted is still unknown although it has been proposed a two steps mechanism in which the myosin motors have a crucial role in the ring contraction while a parallel process of depolymerization of the actin filaments contribute to the the ring shrinking as well [33]. 


\subsection{Mathematical models in wound healing}

Apart from key results in experimental biology, many efforts have been made to propose theoretical and numerical models to describe the mechanical response of tissues to injuries and perform numerical simulations of the process. At the tissue level, considering wound in the epidermis, several computational and mechanistic approaches to the angiogenesis of the process and the healing mechanism have been proposed in the literature $[34,35,36,37]$. At the epithelium scale and for a non-adhesive circular gap, Vedula et. al. [17] developed a mechanical model to analyze the effect of contractile forces of the actin cable against a resistive viscoelastic behavior of the cells. They used a solid viscoelastic Kelvin-Voigt model and a linear adhesive force for the interaction of the epithelium and the substrate. Similarly, Ravasio et. al. [2] did finite element simulations to study the effect of different curvatures in the initial gap over the forces and speeds on the tissue. In their work, interaction between cells were considered viscous as well as the friction with the substrate. The forces acting on the moving boundary were introduced as Neumann boundary conditions, dependent on experimental data and local curvature. Additionally, also applying the ring contraction as a boundary force, Almeida et. al. [38, 39] proposed models that take into account the epidermal tension and the zipping forces in homogeneous wound closure and in non-homogeneous dorsal wound closure using level-set methods to track the tissue edge. They also resorted to genetic algorithms to find the material properties and model constants by qualitative comparison with experimental cases.

The wound closure and performance of surrounding cells, without mitotic processes, was also analyzed computationally in the case of small wounds by Nagai et. al. [40]. Their models represented each cell discretely using vertex dynamics cell model in order to perform wound closure. Brugués et. al. [22], performed wound healing simulations on elastic substrates also using forward vertex analysis. To do so, cell boundaries were discretized using a series of bar elements with viscous properties combined with constant tractions. An area conservation constrain and internal dash-pots for the cytoplasm were imposed to find equilibrium. Wound closure was simulated by imposing a cell contractility at the wound edge (purse string) and lamellipodia protrusions (cell crawling) using also vertex models [22, 41, 42].

The mentioned works in epithelia were able to reproduce experimental results from the laboratory, showing the cell-to-cell mechanics, the cell crawling or the reaction to the actomyosin cable. They were also able to provide valuable information about the closing velocity or forces at different scales of the problem. Unfortunately, none of them takes into account the coupling between the tissue deformation, the effect of chemical signals during the early and late stages of the process or how these chemical signals affect the actin/myosin concentrations and the afterward effect in the active contraction. All these phenomena span a fundamental mechano-chemical sensing at the early stages of wound healing that can be described by means of coupled interaction of elliptical partial derivative equations. Therefore, the interaction of biochemical concentration with the mechanical response of the epithelial layer has never been considered in the modeling of wound healing. In particular, two processes are of profound relevance during wound healing: 1) the early short time contraction of individual epithelial cells as a function of the intracellular actomyosin in the layer [5] and 2) the later contraction of the actin ring which again appears as a function of the actomyosin concentration, now at the wound edge.

In this paper we create a mathematical model of the entire wound healing process to be solved along with finite element schemes. The modeling of both time scale of the problem is intended: one associated with the formation of the actin cable and another associated with later contraction of the purse-string. The tissue is considered as a continuum. For the mechanical description of the problem a non-linear large strain hyperelastic model is used. The transport problem of $\mathrm{Ca}^{2+}$ is defined by a non-linear unsteady diffusionreaction equation. The actomyosin turnover is defined by means of a non-linear source term of the $\mathrm{Ca}^{2+}$ concentration. The mentioned system is analyzed with a coupled system of equations solved implicitly in a monolithic fashion within a finite element method. Once the actomyosin ring is formed, the cable contraction is modeled as an active element, following the classical Hill's description, added to the previous mechanical description. Both processes are solved numerically using an in-house finite element code.

The work is organized as follows. First, the basic continuum problem is described in Section 2, explaining the general kinematic framework, the balance equations governing the system and those constitutive laws 
that govern the problem along the different time and space scales. Second, in Section 3, the numerical approach is presented by defining the strong and weak forms of the coupled system, along with the time and spatial discretization and a consistent linearisation of the resulting system of equations. Later the main mechanical properties of the epithelium are defined as well as any mechanical-related variable need for the finite element simulations, taking into account the particularities in the constitutive relations for the ring formation and purse-ring respectively. Sections 4 and 5 describe two separate mechanisms:

- Actin Ring contraction (Section 4):

- Wound formation (Section 4.1)

- Calcium $(\phi)$ transport and actin $(\rho)$ concentration (Sections 4.2 and 4.3)

- Mechanical contraction (Section 4.4)

- Purse String contraction (Section 5)

- Contraction velocity (Sections 5.1-5.2)

- Consitutive equations and mechanical equilibrium (Section 5.3)

Relevant results of the model are presented in Section 6 along with a final discussion and some conclusions, respectively in sections 7 and 8 .

\section{General framework}

We will present here the general form of the coupled chemo-mechanical problem, which is built from transport equations (Section 2.2) and balance of linear momentum (Section 2.3), which will be described after some preliminary definitions.

\subsection{Kinematic definitions and decomposition of the deformation gradient}

Following [43], the spatial parametrization $\{\bullet\}(\mathbf{x}, \mathrm{t})$ and the material parametrization $\{\bullet\}(\mathbf{X}, \mathrm{t})$ are formulated in terms of the spatial coordinate $\mathbf{x}$ and the material coordinate $\mathbf{X}$ respectively. A distinction is made for a spatial and material reference of a given quantity (scalar or tensor), denoted as $\{\bullet\}_{\mathrm{t}}$ when related to the spatial domain $\Omega$ or $\{\bullet\}_{0}$ related to the material domain $\Omega_{0}$. Any quantity described in the spatial configuration is an element tangent or cotangent to $\Omega$, while if described in the material configuration is tangent or cotangent to $\Omega_{0}$. The problem is restricted to its spatial motion, which consist on following the particles of the body from a fixed material position $\mathbf{X}$. The spatial motion problem is characterized through the map,

$$
\mathbf{x}=\varphi(\mathrm{X}, \mathrm{t}): \quad \Omega_{0} \rightarrow \Omega
$$

from the material placement $\mathbf{X}$ of a particle in $\Omega_{0}$, to the spatial placement $\mathbf{x}$ of the same particle in the spatial configuration $\Omega$. The deformation gradient $\mathbf{F}$ is defined as the spatial derivative of the deformation map $\varphi$ with respect to the reference configuration $\mathbf{X}$ at time $t_{0}$ as

$$
\mathbf{F}=\nabla_{X} \varphi
$$

with determinant $\mathrm{J}=\operatorname{det}(\mathbf{F})$. The Cauchy-Green deformation tensor $\mathbf{C}$ is introduced to define the relevant strain measures as $\mathbf{C}=\mathbf{F}^{t} \cdot \mathbf{F}$. Finally the first strain invariant evaluated for the total deformation tensors as $\mathrm{I}_{1}=\operatorname{tr}(\mathbf{C})$.

We will resort to the multiplicative decomposition of the deformation gradient $\mathbf{F}$ into an elastic part $\mathbf{F}_{\mathrm{e}}$ and an active part $\mathbf{F}_{(\cdot)}[44]$,

$$
\mathbf{F}=\mathbf{F}_{\mathrm{e}} \cdot \mathbf{F}_{(\cdot)}
$$

The underlying concept is adopted from the multiplicative decomposition in finite elastoplasticity [45]. The elastic part represents the original mechanical response of the material, such as the volume changing 
and volume preserving deformations that would result from the loading of the system. The active part is the material response to any other external excitation. In this work we consider two different active responses: volume change that depends on the actin concentration (Section 4), and actomyiosin ring contraction (Section 5).

The determinants of the elastic and growth tensors are denoted by $\mathrm{J}_{\mathrm{e}}=\operatorname{det}\left(\mathbf{F}_{\mathrm{e}}\right)$ and $\mathrm{J}_{(\cdot)}=\operatorname{det}\left(\mathbf{F}_{(\cdot)}\right)$, respectively, such that $\mathrm{J}=\mathrm{J}_{\mathrm{e}} \mathrm{J}_{(\cdot)}$. The elastic right Cauchy Green tensor $\mathbf{C}_{\mathrm{e}}$ can be introduced as

$$
\mathbf{C}_{\mathrm{e}}=\mathbf{F}_{\mathrm{e}}^{\mathrm{t}} \cdot \mathbf{F}_{\mathrm{e}}=\mathbf{F}_{(\cdot)}^{-\mathrm{t}} \cdot \mathbf{C} \cdot \mathbf{F}_{(\cdot)}^{-1}
$$

\subsection{Biochemical Transport Problem}

The transport of a given substance due to diffusion mechanism and a volumetric source term $\mathrm{F}^{u}$ will be here described through the parabolic transport equation

$$
\dot{\mathrm{u}}=\nabla \cdot \mathbf{Q}+\mathrm{F}^{u}(\mathrm{u}, t) \quad \text { in } \Omega,
$$

where $\mathrm{u}$ is the transported quantity, and the transient term is the time derivative $\dot{\mathrm{u}}=d \mathrm{u} / d t[46]$. The volumetric reaction/source-term $\mathrm{F}^{\mathrm{u}}(\mathrm{u}, t)$ represents the creation or destruction of the the given quantity. The first terms on the rhs is a diffusive term, which depends on the divergence of a given flux $\mathbf{Q}$ that is generally a non-linear function of $\mathrm{u}$. The equation has the following Dirichlet and Neumann boundary conditions

$$
\begin{aligned}
\mathrm{u} & =\overline{\mathrm{u}} \quad \text { in } \Gamma_{D}^{u} \\
\mathbf{Q} \cdot \mathbf{N} & =\overline{\mathrm{T}}^{u} \quad \text { in } \Gamma_{N}^{u},
\end{aligned}
$$

being $\bar{u}$ a prescribed quantity in the essential boundary $\Gamma_{D}^{u}$, and $\overline{\mathrm{T}}^{u}$ a prescribed flux on the normal direction $\mathbf{N}$ of the natural boundary $\Gamma_{N}^{u}$. The differential equation is also accompanied by suitable initial conditions.

The process through which intracellular calcium ions are transported through neighboring cells is believed to be through channels located in the cell membrane, known as gap junctions. Gap junctions are assemblies of cell-cell channels, which are formed through the docking of proteins located in the cell membrane [47]. The result of the assembly of this channels is a porous membrane [48] that allows the passing of molecules up to a given size, which is bigger than the calcium ion molecules [47]. In Figure 5 a scheme for the mentioned mechanism can be observed.
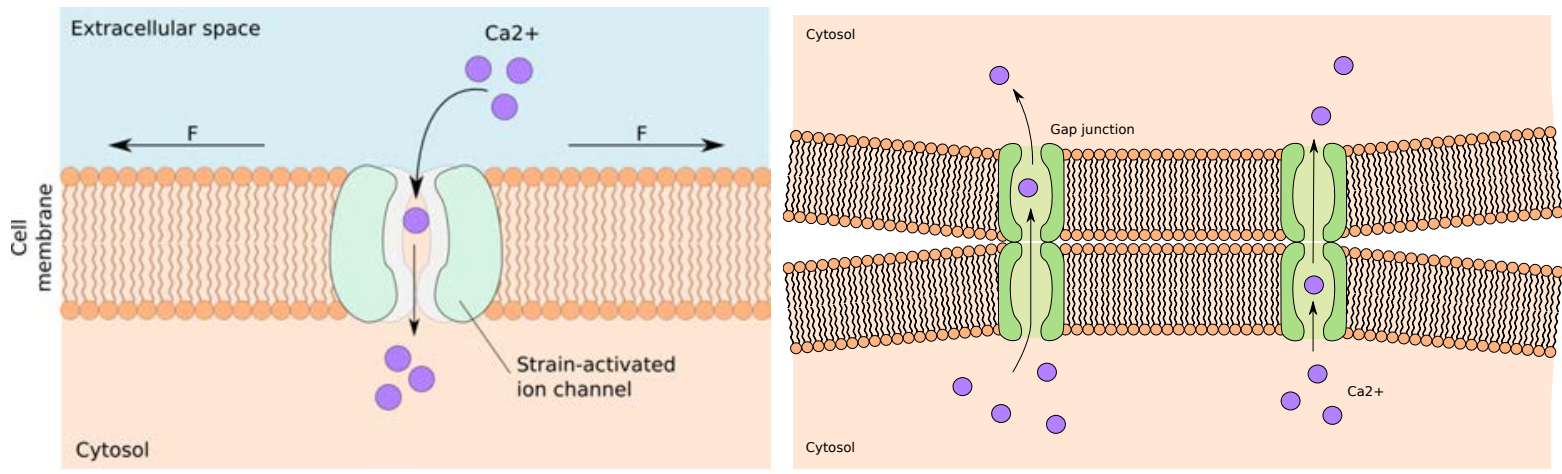

Figure 5: Left: Strain activated calcium ion channel mechanics. The cell membrane, which separates the interior of the cell with the exterior space, regulates its permeability to ion fluxes through proteins that serve as gates. When the membrane is subject to dilatation stretches, the channels open up and allow the flow of calcium ions through the cell membrane. Right: Gap junction channels in cell membranes. The assembly of two hemichannel formed by connexins create a channel through which several substances, calcium ion included, can pass from cell to cell.

The concentration flux $\mathbf{Q}$ describes the natural phenomenon of diffusion, which we will assume proportional to the concentration gradient $\nabla \mathrm{u}$, that is, $\mathbf{Q}=\mathbf{D} \nabla \mathbf{u}$, with $\mathbf{D}$ the diffusion tensor. We adopt a 
constant isotropic behavior, so that the tensor is expressed as a function of only one parameter, $\mathbf{D}=\nu \mathbf{I}$, with $\nu$ the diffusion coefficient,

$$
\mathbf{Q}=\nu \nabla \mathrm{u}
$$

\subsection{Balance of Linear Momentum}

The principle of virtual work in continuum mechanics [49] states that the internal power production $\hat{\mathrm{P}}_{\mathrm{i}}(\boldsymbol{\eta})$ and external power production $\hat{\mathrm{P}}_{\mathrm{e}}(\boldsymbol{\eta})$ should be equal,

$$
\hat{\mathrm{P}}_{\mathrm{i}}(\boldsymbol{\eta})-\hat{\mathrm{P}}_{\mathrm{e}}(\boldsymbol{\eta})=0
$$

being $\boldsymbol{\eta}$ the set of admissible velocities [50,51], and the internal and external power are defined as:

$$
\begin{aligned}
& \hat{\mathrm{P}}_{\mathrm{i}}(\boldsymbol{\eta})=\int_{\Omega} \mathbf{P}: \nabla \boldsymbol{\eta} d \Omega, \text { in } \Omega \\
& \hat{\mathrm{P}}_{\mathrm{e}}(\boldsymbol{\eta})=\int_{\Gamma} \mathbf{T}^{\varphi} \cdot \boldsymbol{\eta} d \Gamma, \text { in } \Gamma_{N}^{\varphi},
\end{aligned}
$$

where $\mathbf{P}$ is the first Piola-Kirchoff stress tensor, $\mathbf{T}^{\varphi}$ is the material traction vector and external body forces have been neglected. Applying the divergence theorem to (8), it yields the well known balance of linear momentum. Due to the low mass and velocities, inertial and transient terms are also neglected in the balance, which adopts the following quasi-static form:

$$
\mathbf{0}=\nabla \cdot \mathbf{P}, \quad \text { in } \Omega .
$$

This equation has the following Dirichlet and Neumann boundary conditions:

$$
\begin{aligned}
\varphi & =\bar{\varphi}, \quad \text { in } \Gamma_{D}^{\varphi}, \\
\mathbf{P}^{\mathrm{t}} \cdot \mathbf{N} & =\overline{\mathbf{T}}^{\varphi}, \quad \text { in } \Gamma_{N}^{\varphi},
\end{aligned}
$$

where $\bar{\varphi}$ and $\overline{\mathbf{T}}^{\varphi}$ are the prescribed displacements and external forces respectively. Henceforth, only Dirichlet mechanical boundary conditions are strongly imposed in the exterior of the domain, setting the displacement to zero: $\varphi=0$ in $\Gamma_{D}^{\varphi}$, and no Neumann boundary condition is imposed.

\subsection{Hyperelastic constitutive equations}

In order to describe the hyperelastic behaviour of the material, the constitutive equation is obtained directly from the Clausius-Planck form of the second law of thermodynamics [52], reading

$$
\mathcal{D}_{\text {int }}=\frac{1}{2} \mathbf{S}: \dot{\mathbf{C}}-\dot{\Psi}-\mathrm{S}_{0} \theta=\left[\mathbf{S}-2 \partial_{\mathbf{C}} \Psi\right]: \dot{\mathbf{C}}-\mathrm{S}_{0} \theta \geq 0
$$

where $\mathcal{D}_{\text {int }}$ is the dissipation of internal energy, $\mathbf{S}$ is the second Pila-Kirchoff stress tensor, $\dot{\mathbf{C}}$ is the time derivative of the right Cauchy-Green strain tensor, $\dot{\Psi}$ is the time derivative of the strain energy density function $(\mathrm{SEDF})$ and $\mathrm{S}_{0} \theta$ is the material form of the entropy. Note, that in this paper, a purely mechanical theory is used, so that thermal effects are ignored ( $S_{0}$ and $\theta$ are omitted), and a perfectly elastic material is considered [50]. In this case the inequality reads

$$
\left(\mathbf{S}-2 \partial_{\mathbf{C}} \Psi\right): \dot{\mathbf{C}}=0 .
$$

Due to the arbitrariness of $\mathbf{C}$ and hence $\dot{\mathbf{C}}$, the expression in parenthesis should be null, and the well known expression for the second Piola-Kirchoff is retrieved

$$
\mathbf{S}=2 \frac{\partial \Psi}{\partial \mathbf{C}}
$$


For the finite element implementation, the first Piola-Kirchoff is used, which is obtained by pulling back tensor $\mathbf{S}$ to the reference configuration as $\mathbf{P}=\mathbf{F} \cdot \mathbf{S}$. Alterantively, $\mathbf{P}$ can be also derived as a function of the SEDF, considering the dissipation equation as a function of $\mathbf{P}$ and $\mathbf{F}$ instead of $\mathbf{S}$ and $\mathbf{C}$ [50], resulting in $\mathbf{P}=\partial_{\mathbf{F}} \Psi$.

If the problem is defined in terms of the multiplicative decomposition of the deformation gradient, then $\Psi=\Psi\left(\mathbf{C}_{e}, \mathbf{F}_{(\cdot)}\right)$ and the Piola-Kirchoff expression can be further defined, resorting to equation (3), as

$$
\mathbf{S}=2 \frac{\partial \Psi}{\partial \mathbf{C}}=2 \frac{\partial \Psi}{\partial \mathbf{C}_{e}}: \frac{\partial \mathbf{C}_{e}}{\partial \mathbf{C}}=\mathbf{F}_{(\cdot)}^{-1} \mathbf{S}_{e} \mathbf{F}_{(\cdot)}^{-t} \quad \text { with } \quad \mathbf{S}_{e}=2 \frac{\partial \Psi}{\partial \mathbf{C}_{e}}
$$

\subsection{Baseline elasticity}

We adopt a classical Neo-Hookean SEDF with a constitutive law that reads

$$
\Psi(\mathbf{C})=\frac{1}{2} \lambda \ln ^{2}(\mathrm{~J})-\mu \ln (\mathrm{J})+\frac{1}{2} \mu\left(\mathrm{I}_{1}-3\right),
$$

where $\lambda$ and $\mu$ are the Lamé parameters which describe de mechanical properties of the material, and $\mathrm{J}=\operatorname{det}(\mathbf{F})$ and $\mathrm{I}_{1}=\operatorname{trace}(\mathbf{C})$. The first Piola-Kirchoff stress tensor is derived from (13) and (14) as,

$$
\mathbf{P}=\mu \mathbf{F}+(\lambda \ln (\mathrm{J})-\mu) \mathbf{F}^{-t}
$$

\section{Finite element formulation of the coupled mechanical-transport equations}

The partial differential equations described in the previous section are here discretized in time and in space using the finite element method in order to solve the boundary value problem numerically. As the problem of interest is coupled and non-linear, the resulting weak forms are linearized with respect to the involved variables with the objective of using a Newton-Raphson method.

\subsection{Weak form}

First, the strong form of the continuous equations valid for the domain $\Omega$, in their residual form are identified

$$
\begin{aligned}
& \mathrm{R}^{\mathrm{u}}:=\dot{\mathrm{u}}-\nabla \cdot \mathbf{Q}-\mathrm{F}^{\mathrm{u}}=0 \quad \text { in } \Omega, \\
& \mathbf{R}^{\varphi}:=-\nabla \cdot \mathbf{P}-\mathbf{F}^{\varphi}=0 \quad \text { in } \Omega,
\end{aligned}
$$

with Dirichlet and Neumann boundary conditions.

Recalling the usual Hilbert spaces [46] we define:

$$
\begin{array}{ll}
\mathcal{S}^{u}=\left\{\mathrm{u} \in \mathcal{H}^{1}(\Omega) \mid \mathrm{u}=\overline{\mathrm{u}} \text { on } \Gamma_{D}^{u}\right\}, & \mathcal{V}^{u}=\left\{\delta \mathrm{u} \in \mathcal{H}^{1}(\Omega) \mid \delta \mathrm{u}=0 \text { on } \Gamma_{D}^{u}\right\}, \\
\mathcal{S}^{\varphi}=\left\{\boldsymbol{\varphi} \in\left[\mathcal{H}^{1}(\Omega)\right]^{d} \mid \boldsymbol{\varphi}=\bar{\varphi} \text { on } \Gamma_{D}^{\varphi}\right\}, & \mathcal{V}^{\varphi}=\left\{\delta \boldsymbol{\varphi} \in\left[\mathcal{H}^{1}(\Omega)\right]^{d} \mid \delta \boldsymbol{\varphi}=\mathbf{0} \text { on } \Gamma_{D}^{\varphi}\right\},
\end{array}
$$

with $d$ the space dimensions. In order to obtain the weak form of (16) and (17), integration by parts and multiplication with two test functions $\delta \mathrm{u}$ and $\delta \varphi$ is carried out, resulting in the following weak form of the reaction-diffusion and balance of momentum respectively:

Find $\mathbf{u} \in \mathcal{S}^{\mathrm{u}}$ and $\varphi \in \mathcal{S}^{\varphi}$ such that,

$$
\begin{aligned}
\mathrm{G}^{\mathrm{u}}=\int_{\Omega} \delta \mathrm{u} \cdot \dot{\mathrm{u}} \mathrm{d} \Omega+\int_{\Omega} \nabla \delta \mathrm{u} \cdot \mathbf{Q} \mathrm{d} \Omega-\int_{\Gamma_{Q}} \delta \overline{\mathrm{T}}^{u} \mathrm{~d} \Gamma-\int_{\Omega} \delta \mathrm{uF} \mathrm{F}^{u} \mathrm{~d} \Omega=0, & \forall \delta \mathrm{u} \in \mathcal{V}^{\mathrm{u}}, \\
\mathrm{G}^{\varphi}=\int_{\Omega} \nabla_{\mathbf{X}} \delta \boldsymbol{\varphi}: \mathbf{P} \mathrm{d} \Omega-\int_{\Gamma_{P}} \delta \boldsymbol{\varphi} \cdot \overline{\mathbf{T}}^{\varphi} \mathrm{d} \Gamma=0, & \forall \delta \boldsymbol{\varphi} \in \mathcal{V}^{\varphi} .
\end{aligned}
$$




\subsection{Time discretization}

To carry out the temporal discretization, a first order Backward-Euler implicit scheme is used to solve the time integration problem. The temporal discretization is given by a time interval $\mathcal{T}$ and $\mathrm{N}$ subintervals, $\mathcal{T}=\bigcup_{0}^{N}\left[\mathrm{t}_{n}, \mathrm{t}_{n+1}\right]$ with a constant time increment $\Delta \mathrm{t}=\mathrm{t}_{n+1}-\mathrm{t}_{n} \geq 0$. The discretization of $\dot{\mathrm{u}}$ from (18) takes the form

$$
\dot{\mathrm{u}}=\frac{\mathrm{u}-\mathrm{u}_{n}}{\Delta t},
$$

where $\mathrm{u}$ refers to the displacement at the current time step $t_{n+1}$, and $\mathrm{u}_{n}$ at the previous one, $t_{n}$. Note that the mechanical part also follows an implicit scheme, but due to the quasi-static considerations of (9), variable $\varphi$ refers always to the current time step.

\subsection{Spatial discretization}

For the discretization of the spatial domain $\Omega$, a $\mathrm{C}^{0}$ Lagrangian basis is taken in order to interpolate all the variables, introducing them as global degrees of freedom at the nodal level. Using the isoparametric concept, same shape functions $N^{u}$ and $N^{\varphi}$ are used both for the geometry representation and the approximation functions $\mathrm{u}^{h}$ and $\varphi^{h}$. Galerkin method is used in order to approximate the test functions $\delta \mathrm{u}^{h}$ and $\delta \varphi^{h}$. Then, the approximations read

$$
\begin{aligned}
\left.\delta \mathrm{u}^{h}\right|_{\Omega^{e}} & =\sum_{i=1}^{n_{e u}} N_{i}^{u} \delta \mathrm{u}_{i}, & \left.\delta \varphi^{h}\right|_{\Omega^{e}} & =\sum_{j=1}^{n_{e \varphi}} N_{j}^{\varphi} \delta \varphi_{j} \\
\left.\mathrm{u}^{h}\right|_{\Omega^{e}} & =\sum_{k=1}^{n_{e u}} N_{k}^{u} \mathrm{u}_{k}, & \left.\varphi^{h}\right|_{\Omega^{e}} & =\sum_{l=1}^{n_{e u}} N_{l}^{\varphi} \varphi_{l},
\end{aligned}
$$

where $\Omega^{\mathrm{e}}$ defines the element volume such that $\Omega=\cup_{\mathrm{e}=1}^{\mathrm{n}_{\mathrm{el}}} \Omega^{\mathrm{e}}$ and $n_{e u}$ and $n_{e \varphi}$ are the number of nodes per element for each approximation. Note that in this case, the same bilinear element is used for the approximation of both variables, and therefore $n_{e u}=n_{e \varphi}$ and $N_{i}^{u}=N_{i}^{\varphi}$.

We can rewrite (18) and (19) using the approximations (20)-(22) to obtain the time/space discretization of the weak residual vector form as

$$
\begin{aligned}
& \mathrm{R}_{i}^{u}:={\underset{e=1}{n_{e l}}}_{\int_{\Omega^{e}}} N_{i}^{u} \frac{1}{\Delta t}\left(\mathrm{u}-\mathrm{u}_{n}\right) \mathrm{d} \Omega+\int_{\Omega^{e}} \nabla N_{i}^{u} \cdot \mathbf{Q} \mathrm{d} \Omega-\int_{\Omega^{e}} N_{i}^{u} \mathrm{~F}^{u} \mathrm{~d} \Omega-\int_{\Gamma_{Q}^{e}} N_{i}^{u} \overline{\mathrm{T}}^{u} \mathrm{~d} \Gamma=0 \\
& \mathbf{R}_{j}^{\varphi}:=\AA_{e=1}^{n_{e l}} \int_{\Omega^{e}} \nabla N_{j}^{\varphi} \cdot \mathbf{P} \mathrm{d} \Omega-\int_{\Gamma_{P}^{e}} N_{j}^{\varphi} \overline{\mathbf{T}}^{\varphi} \mathrm{d} \Gamma=0,
\end{aligned}
$$

where the operator $\mathbf{A}$ symbolizes the assembly of the local contributions to the global residual equation. The non-linear equations in (23)-(24) are solved resorting to a Newton-Raphson iterative process. This process, and the required linearized forms of the residuals are given in Section 3.4.

\subsection{Newton-Raphson solution process and linearization of residuals}

We solve the problem stated in (23)-(24) with a monolithic incremental iterative Newton-Raphson strategy. A consistent linearization of the residual equations is required. At time $t_{n+1}$ the latter read,

$$
\begin{aligned}
& \mathrm{R}_{i(n+1)}^{u(k+1)}=\mathrm{R}_{i(n+1)}^{u(k)}+\mathrm{dR}_{i}^{u}=0, \\
& \mathbf{R}_{j(n+1)}^{\varphi(k+1)}=\mathbf{R}_{j(n+1)}^{\varphi(k)}+\mathrm{d} \mathbf{R}_{j}^{\varphi}=\mathbf{0},
\end{aligned}
$$


where $k$ is the iteration number and the residual variations are given by

$$
\begin{aligned}
\mathrm{dR}_{i}^{u} & =\sum_{k} \mathbf{k}_{i k}^{u u} \mathrm{~d} u_{k}+\sum_{l} \mathbf{K}_{i l}^{u \varphi} \mathrm{d} \boldsymbol{\varphi}_{l}, \\
\mathrm{~d} \mathbf{R}_{j}^{\varphi} & =\sum_{K} \mathbf{K}_{j k}^{\varphi u} \mathrm{~d} u_{k}+\sum_{l} \mathbf{K}_{j l}^{\varphi \varphi} \mathrm{d} \boldsymbol{\varphi}_{l} .
\end{aligned}
$$

Here, the unknowns $d u_{K}$ and $d \varphi_{l}$ are the updates of the transport and mechanical variables and matrices $\mathbf{K}$ are the result of deriving the residual equations (23) and (24) with respect to both unknowns $\mathrm{u}$ and $\boldsymbol{\varphi}$. The solution of the system of equations (25) renders the iterative update for the increments of the global unknowns $\mathrm{u}_{i} \leftarrow \mathrm{du} \mathrm{u}_{i}$ and $\boldsymbol{\varphi}_{j} \leftarrow \mathrm{d} \boldsymbol{\varphi}_{j}[53]$.

After assuming that the boundary conditions are independent of the solution of the problem at each time-step, the following Jacobian of the residuals are obtained,

$$
\begin{aligned}
& \mathbf{K}_{i k}^{u u}=\frac{\mathrm{dR}_{i}^{u}}{\mathrm{~d} u_{k}}=\stackrel{n_{e l}}{\mathbf{A}} \int_{\Omega^{e}}\left(N_{i}^{u} \frac{1}{\Delta t} N_{k}^{u}-N_{i}^{u} \mathrm{~d}_{u} \mathrm{~F}^{u} N_{k}^{u}+\nabla N_{i}^{u} \mathrm{~d}_{\nabla u} \mathbf{Q} \cdot \nabla N_{k}^{u}\right) \mathrm{d} \Omega, \\
& \mathbf{K}_{i l}^{u \varphi}=\frac{\mathrm{dR}_{i}^{u}}{\mathrm{~d} \boldsymbol{\varphi}_{l}}={\stackrel{\text { A }}{n_{e l}}}_{e=1} \int_{\Omega^{e}}\left(N_{i}^{u} \cdot d_{\mathbf{F}} \mathrm{F}^{u} \nabla N_{l}^{\varphi}+\nabla N_{i}^{u} \cdot \mathrm{d}_{\mathbf{F}} \mathbf{Q} \cdot \nabla N_{l}^{\varphi}\right) \mathrm{d} \Omega, \\
& \mathbf{K}_{j k}^{\varphi u}=\frac{\mathrm{dR}_{j}^{\varphi}}{\mathrm{d} \varphi_{k}}=\stackrel{n_{e=1}}{\AA} \int_{\Omega^{e}}\left(\nabla N_{j}^{\varphi} \cdot \mathrm{d}_{u} \mathbf{P} N_{k}^{u}\right) \mathrm{d} \Omega \\
& \mathbf{K}_{j l}^{\varphi \varphi}=\frac{\mathrm{dR}_{j}^{\varphi}}{\mathrm{d} \varphi_{l}}=\stackrel{n_{e l}}{\mathbf{A}} \int_{\Omega^{e}}\left(\nabla N_{j}^{\varphi} \cdot \mathrm{d}_{\mathbf{F}} \mathbf{P} \nabla N_{l}^{\varphi}\right) \mathrm{d} \Omega .
\end{aligned}
$$

The specific forms of the linearized terms are provided in following sections. In equation (26), the mass matrix, the reaction matrix and the diffusive matrix can be identified, while in equation (29) the component $d_{\mathbf{F}} \mathbf{P}$ is the classical elastic tangent operator $\mathbb{A}$, which under the assumed hyperelastic constitutive relation, is given by

$$
\mathbb{A}=\lambda \mathbf{F}^{-\mathrm{t}} \otimes \mathbf{F}^{-\mathrm{t}}-(\lambda \ln (\mathrm{J})-\mu) \mathbf{F}^{-\mathrm{t}} \underline{\otimes} \mathbf{F}^{-1}+\mu \mathbf{I} \bar{\otimes} \mathbf{I},
$$

with the special tensor products $[\bullet \bar{\otimes} \circ]_{i j k l}=(\bullet)_{i k}(\circ)_{j l}$ and $[\bullet \otimes \circ \circ]_{i j k l}=(\bullet)_{i l}(\circ)_{j k}$.

The other two terms $\mathbf{K}_{i l}^{u \varphi}$ and $\mathbf{K}_{j k}^{\varphi u}$ are the result of deriving the transport and mechanical residual with respect to the mechanical and transport variables respectively. In equation (27), terms $\mathrm{d}_{\mathbf{F}} \mathrm{F}^{u}$ and $\mathrm{d}_{\mathbf{F}} \mathbf{Q}$ describes the variation of the source term and flux with respect to the deformation gradient, and in equation (28), $d_{u} \mathbf{P}$ describes the variation of the material stress response to the transported quantity concentration. Note that in case that the systems were uncoupled, this terms would be null, and two separate systems would be obtained.

\section{Modeling of actin ring formation}

In this section, the model adopted for the first stage of the wound healing is presented, which takes part on the first 15 minutes of the overall process. The initial expansion of injury and the formation of the actin ring are described.

\subsection{Wound formation}

Before the wound is induced, there exists a pre-strain which is here modeled with an initial deformation gradient $\mathbf{F}_{0}$. When the ablation is produced with a laser, tissue retracts, and the wound gap expands, creating an area of high tension and strain in the border of the injury.

In order to impose the pre-strain, an initial isotropic deformation gradient is incorporated multiplicatively into the global deformation gradient $\mathbf{F}$, at every point of the domain as

$$
\mathbf{F}_{e}=\mathbf{F F}_{0}^{-1} \quad \text { with } \quad \mathbf{F}_{0}=\mathrm{f}_{\text {pre }} \mathbf{I},
$$


where the initial scalar stretch factor $f_{\text {pre }} \leq 1$. Note, that the tissue in the reference configuration is not loaded. It is also important to mention, that the governing equations used for this stage are the same as in the rest of the ring formation process, which are described in sections 4.2, 4.3 and 4.4, but the time-scale varies, being fractions of seconds for the wound expansion and minutes for the processes that generate the actomyosin ring.

\subsection{Calcium burst and transport}

Due to the tissue deformation, the ion channels, which are activated through cell's strain, open and let ions of extracellular calcium enter the cytosol. Once the calcium diffuses inside the cell, it propagates to the neighbor cells through a pure diffusion mechanism [24]. In order to account for such phenomena, the diffusion-reaction equation presented in Section 2.2 will be used to characterize the calcium transport mechanism. This is here represented with the scalar variable $\phi$ as the calcium concentration and primary unknown of the problem, which replaces unknown $u$ in the transport equation (4). For the purpose of this model, a constant uniform diffusion coefficient $\nu$ is introduced into the calcium transport equation in order to reduce the complexities of the non-linear term by including still partially unknown biological responses. Its value is set to the order of the values obtained in Long et. al. [24] .

Studies over mechanosensitive gating, propose different formulas to obtain the probability of the channel opening depending on the applied tension on the tissue, among other parameters [54]. The simpler one, the Sackin's formula, relies on the linear elasticity of the tissue elements, resulting on a opening trigger energy with a quadratic dependence of the tension $[55,56,54]$ :

$$
\mathrm{p}_{0}(\mathrm{~T})=\frac{\mathrm{p}_{\max }}{1+\mathrm{ke}^{-\tau \mathrm{T}^{2}}}
$$

where $\mathrm{p}_{0}$ is the single channel open probability, $\mathrm{p}_{\max }$ is the maximum possible value, $\mathrm{k}$ is a pressure independent constant, $\tau$ is the stretch sensitivity of the membrane and $\mathrm{T}$ is the applied tension [54]. The resulting function is of sigmoidal type, setting a maximum and minimum for the probability, with a controlled slope in between, for different suction tensions.

Formula in (32) depends on a suction tension applied in very particular conditions. Here, we propose an analogous sigmoidal function to directly determine the source term as a function of the first strain invariant $\mathrm{I}_{1}=\operatorname{tr}(\mathbf{C})$. Additionally, this function is multiplied by a reactive term, that limits the introduction of calcium to the cell when a given concentration is already reached. This is done in order to restrain the maximum possible creation of calcium as there cannot be an unlimited amount of calcium in the cells and to keep the concentration in the order of what was observed experimentally [5]. Consequently, we will use the following form of the source term

$$
\mathrm{F}^{\phi}=\mathrm{F}_{1}\left(\mathrm{I}_{1}\right) \mathrm{F}_{2}(\phi)
$$

where function $\mathrm{F}_{1}\left(\mathrm{I}_{1}\right)$, analogously to (32), has the particularity of being upper and lower bounded and does not generate calcium when compression strains are applied to the material. It takes the form

$$
\mathrm{F}_{1}\left(\mathrm{I}_{1}\right)=\mathrm{F}_{\min }+\frac{\alpha}{1+\mathrm{e}^{-\beta\left(\mathrm{I}_{1}-\gamma\right)}}
$$

with $\alpha=\mathrm{F}_{\max }-\mathrm{F}_{\min }$ and $\mathrm{F}_{\max }$ and $\mathrm{F}_{\min }$ stand for the maximum and minimum calcium creation rates, $\beta$ accounts for the tissue sensibility and $\gamma$ is a parameter to adjust the strain that triggers the calcium generation.

Regarding the reactive function $\mathrm{F}_{2}(\phi)$, its shape is determined to be maximum when the concentration of calcium, relative to the initial one $\phi_{0}$, is $\phi / \phi_{0}=1$ and minimum when a limit concentration is reached $\phi / \phi_{0}=\phi_{\mathrm{lim}}^{*}$, ensuring that the relative concentration is always $1 \leq \phi / \phi_{0} \leq \phi_{\mathrm{lim}}^{*}$. A quadratic function is chosen, for practical implementation reasons, although other shapes would have a similar effect such as a sigmoid or a heaviside function. The proposed function is

$$
\mathrm{F}_{2}(\phi)=1-\phi^{* 2} / \phi_{\lim }^{* 2} \quad \text { with } \phi^{*}=\phi / \phi_{0} .
$$


The result of multiplying $\mathrm{F}_{1}\left(I_{1}\right)$ by $\mathrm{F}_{2}(\phi)$ is a scaling of the source term maximum depending on the current concentration, and therefore controlling both the exterior/interior calcium interchange rate and the maximum allowed calcium. In Figure 6 the changing shape of $F^{\phi}$ for different values of $\phi_{\lim }^{*}$ can be observed, along with the effect over the source term for different combinations of strains and concentrations.

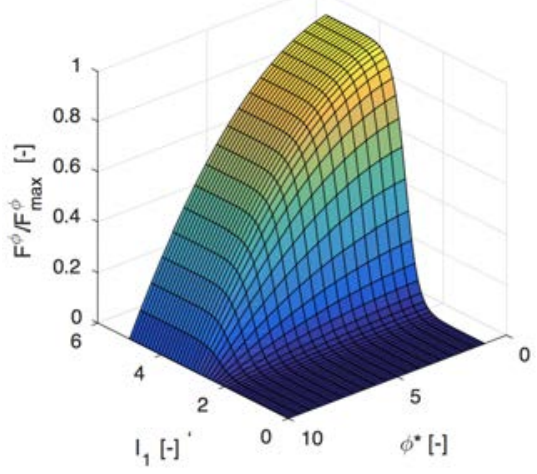

Figure 6: Resulting source term for different hypothetical values of $\mathrm{I}_{1}$ and $\phi^{*}$. Function $\mathrm{F}_{2}$ can be identified in planes for constant $\mathrm{I}_{1}$, while the sigmoidal shape of $\mathrm{F}_{1}$ can be seen in planes for constant $\phi^{*}$. The maximum source term is achieved when the calcium concentration equals the original one and the strain is high.

In short:

- A large diffusion coefficient $\nu$ produces a smoother distribution of calcium and a wider actin ring.

- A large $\mathrm{F}_{\max }^{\phi}$ results in a faster achievement of the maximum calcium distribution.

- A small value of $\beta$ produces calcium creation in a wider area, which can prevent the formation of the actomyosin ring.

- A large $\gamma$ concentrates the creation of calcium around the wound.

The derivatives in (26) to (27) depend on the adopted model for the diffusive source-term $\mathrm{F}^{u}$ (which in the calcium transport corresponds to $\mathrm{F}^{\phi}$ ) and flux $\mathbf{Q}$. After using the source term $\mathrm{F}^{\phi}$ in (33)-(35), it can be deduced that,

$$
\begin{aligned}
\mathrm{d}_{\phi} \mathrm{F}^{\phi} & =-2 \frac{\phi}{\left(\phi_{\lim }^{*} \phi_{0}\right)^{2}}\left(\mathrm{~F}_{\min }+\frac{\alpha}{1+\mathrm{e}^{-\beta\left(\mathrm{I}_{1}-\gamma\right)}}\right), \\
\mathrm{d}_{\mathbf{F}} \mathrm{F}^{\phi} & =\frac{\alpha \beta \mathrm{e}^{-\beta\left(\mathrm{I}_{1}-\gamma\right)}}{\left(\mathrm{e}^{-\beta\left(\mathrm{I}_{1}-\gamma\right)}+1\right)^{2}} 2\left(1-\frac{\phi^{* 2}}{\phi_{\lim }^{* 2}}\right) \mathbf{F} .
\end{aligned}
$$

Since the flux is isotropic, that is $\mathbf{Q}=\nu \nabla \phi$, it is evident that

$$
\begin{aligned}
\mathrm{d}_{\nabla \phi} \mathbf{Q} & =\nu \mathbf{I}, \\
\mathrm{d}_{\mathbf{F}} \mathbf{Q} & =\mathbf{0} .
\end{aligned}
$$

\subsection{Actin filaments and myosin motors recruitment}

According to Antunes et. al [5], the proliferation of actin filaments starts around $20 \mu m$ away from the wound border and there exist a given concentration of calcium at which this phenomenon occurs. Note, that actin filament creation and transport is different from calcium transport, since the former is unable to diffuse across the cell walls. That is why, its behavior is modeled by setting $\nu=0$, and using a simple source term driving the change of the actin concentration $\rho$, i.e.

$$
\dot{\rho}=\mathrm{F}^{\rho} .
$$

As there is no need to account for the diffusive term, the update is done at the integration point level following an explicit Euler method. Since actin concentration is not considered for the calculation of the 
residuals in the finite element implementation, its derivative with respect to the unknown variables are not required.

The source term $\mathrm{F}^{\rho}$ was designed to be different from zero only when the relative calcium concentration is enough and not excesive to allow the actin recruitment:

$$
\mathrm{F}^{\rho}=\mathrm{F}_{\max }^{\rho} \mathrm{e}^{\frac{-\left(\phi^{*}-\epsilon\right)^{2}}{2 \delta^{2}}} \quad \text { with } \phi^{*}=\phi / \phi_{0}
$$

The maximum source is set by parameter $\mathrm{F}_{m a x}^{\rho}$, and the position of the source curve $\mathrm{F}^{\rho} / \mathrm{F}_{\max }^{\rho}$ is controled by the maximum and minimum calcium concentrations at which the actin recruitment is possible, which is set by parameter $\epsilon=\frac{1}{2}\left(\phi^{h i}+\phi^{l o}\right)$. The slope of the curve is adjusted by parameter $\delta$. Figure 7 shows some examples of curves for different values of the parameters $\delta$ and $\epsilon$. In short:

- Parameter $\phi_{\text {lim }}^{*}$ controls the maximum calcium concentration, affecting the velocity of ring formation.

- Small values of $\mathrm{F}_{\max }^{\rho}$ means a slower formation of the actin ring.

- A large value of $\delta$ means a wider actin recruitment area. If it is excessive, it can lead to actin formation in all the domain.

- Parameter $\epsilon$ controls the position of the actin recruitment region. By choosing its value the actin generation region can be set to be steady in the wound border.

Figure 7: Actin source term (variation of $\delta$ and $\epsilon$ ). Actin diffusion source term, scaled up with the maximum possible value, for different combinations of $\delta$ and $\epsilon$. Left: different resulting source term functions with varying slope parameter $\delta$. Lower $\delta$ result in steeper slopes, reducing the region of relative calcium at which the actin recruitment is possible. Right: Different values for $\epsilon$ result in different possitions for the curve. As $\epsilon$ increases, the calcium bounds for the actin recruitment also increases.
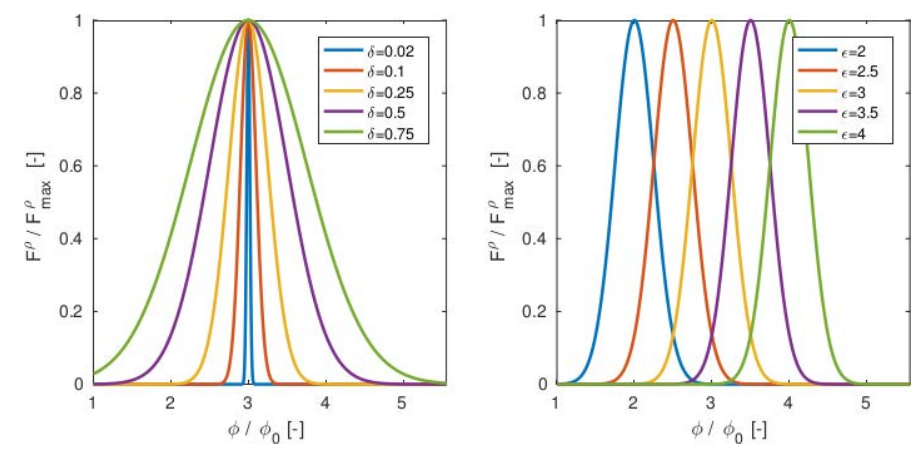

Remark 1 (Myosin motor concentration). Although Antunes et. al. [5] observed a constant space time correlation between the waves of actin and myosin concentration in their experiments, for this model only the actin concentration will be taken into account, as the delay between the two quantities is small compared to the global time scales of the problem. Even if the contraction mechanism has been adjudged to the interaction of myosin motors with actin filaments, the result of overlapping both concentrations would give a similar curve to the one we obtain by evaluating the actin concentration alone but with a slight delay. Therefore, from now on, the actin curve is considered as the actomyosin curve for practical reasons.

\subsection{Active mechanical contraction}

It was observed experimentally that actomyosin ring formation is accompanied by an area reduction in the inflicted wound [5]. In order to reproduce this phenomenon, an active contraction in the tissue was introduced locally, as a function of the actin concentration. To do so, a multiplicative decomposition of the deformation gradient is performed, as stated in equation (2) and similarly to Section 4.1, where the arbitrary component $\mathbf{F}_{(\cdot)}$ takes the form of a shrinking tensor $\mathbf{F}_{g}$, reading

$$
\mathbf{F}=\mathbf{F}_{e} \mathbf{F}_{g} \quad \text { with } \quad \mathbf{F}_{g}=\vartheta \mathbf{I} .
$$

In fact, $\mathbf{F}_{g}$ introduces an isotropic shrinking deformation on each element, determined by a scalar valued variable $\vartheta$. Note that the resulting tensor $\mathbf{F}_{g}$ and the deformation it imposes is transient and it is only relevant as long as the actin concentration in the given point is high enough to produce a shrinking $\vartheta<1$. 
A dependence of the shrinking variable on the relative actin concentration $\rho^{*}=\rho / \rho_{0}$ is proposed, as stated in the following law

$$
\vartheta(\rho)=1-\frac{1-\vartheta_{\min }}{1+\mathrm{e}^{-\tau\left(\rho^{*}-\omega\right)}} .
$$

The sigmoid function is used to characterize the mechanical response towards the actin concentration, with $\vartheta_{\text {min }}$ the minimum expected contraction, which is set to match the contractions observed in the experiments [5], $\tau$ is the sensitivity of the tissue and $\omega$ is a parameter which accounts for the actin concentration at which there exists contraction. In Figure 8, the shape of function $\vartheta(\rho)$ depending on the variation of parameters $\vartheta_{\min }, \tau$ and $\omega$, can be observed. In short:

- Small values of the shrinking variable $\vartheta_{\min }$ lead to more mechanical contraction, which can affect the calcium distribution.

- Large values of $\omega$ mean that more actin concentration is needed to achieve the responsive tissue contraction.

- Small values of $\tau$ yield a smoother interface between contracted and non-contracted regions.
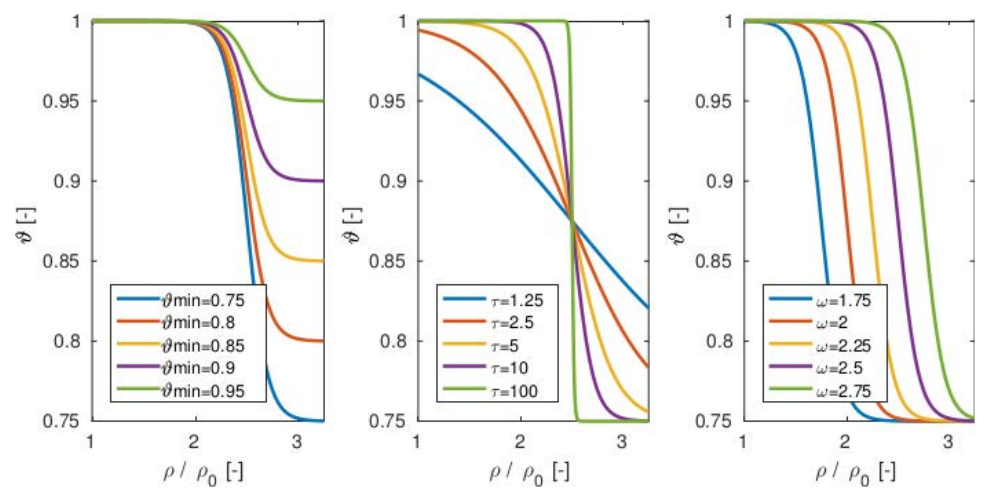

Figure 8: Shrinking variable as a function of $\vartheta_{\min }, \tau$ and $\omega$. Left: with $\vartheta_{\max }=1.0$ smaller minimums are obtained when $\vartheta_{\text {min }}$ is smaller. Center: the tissue sensitivity $\tau$ determines the slope of the curve. As the parameter becomes larger a sharper differentiation between contraction and no contraction regions is observed. Right: the parameter $\omega$ determines the trigger value at which contraction occurs, being this one smaller when the value is small.

Once the modified tensor is calculated at integration point level as $\mathbf{F}_{e}=\mathbf{F F}{ }_{g}^{-1}$, the first Piola-Kirchoff stress $\mathbf{P}$ needed for the evaluation of residual (24) can be calculated by deriving the SEFD equation for hyperelasticity (14) or by evaluating the second Piola-Kirchoff stress $\mathbf{S}$ and performing a pull back with $\mathbf{F}$. Next, we deduce the derivative of $\mathbf{P}$ with respect to the concentration of calcium $\phi$. Note that due to the explicit computation of the actin concentration, there is no direct coupling between the shrinking tensor and the calcium concentration and therefore $\mathrm{d}_{\phi} \mathbf{P}$ is null. The derivative of $\mathbf{P}$ with respect to the deformation gradient $\mathbf{F}, \mathrm{d}_{\mathbf{F}} \mathbf{P}$, may be obtained from the overall tangent operator $\mathbb{C}=2 \mathrm{~d}_{\mathbf{C}} \mathbf{S}$, that is, the derivative of the second Piola-Kirchoff stress tensor with respect to the Cauchy-Green deformation tensor $\mathbf{C}$,

$$
\mathbb{C}=2 \mathrm{~d}_{\mathbf{C}} \mathbf{S}=\mathbb{C}_{\mathrm{e}}+\mathbb{C}_{g}=\left.2 \partial_{\mathbf{C}} \mathbf{S}\right|_{\mathbf{F}_{g}}+\left.2 \partial_{\mathbf{C}} \mathbf{S}\right|_{\mathbf{F}}=\left.2 \partial_{\mathbf{C}} \mathbf{S}\right|_{\mathbf{F}_{g}}+\left.2\left[\partial_{\mathbf{F}_{g}} \mathbf{S}: \partial_{\vartheta} \mathbf{F}_{g}\right] \otimes \partial_{\mathbf{C}} \vartheta\right|_{\mathbf{F}}
$$

The first term of equation (41) represents the classical elastic tangent moduli, $2 \mathrm{~d}_{\mathbf{C}_{\mathrm{e}}} \mathbf{S}_{\mathrm{e}}$, pulled back to the undeformed reference configuration,

$$
\mathbb{C}_{\mathrm{e}}=\left.2 \mathrm{~d}_{\mathbf{C}} \mathbf{S}\right|_{\mathbf{F}_{g}}=\left[\mathbf{F}_{g}^{-1} \bar{\otimes} \mathbf{F}_{g}^{-1}\right]: 2 \mathrm{~d}_{\mathbf{C}_{\mathrm{e}}} \mathbf{S}_{\mathrm{e}}:\left[\mathbf{F}_{g}^{-t} \bar{\otimes} \mathbf{F}_{g}^{-t}\right] .
$$

The second term of equation (41) is zero since the shrinking tensor is not dependent on the deformation gradient.

\section{Modeling of the purse-string contraction}

In this section, the model adopted for the second stage of the wound healing process is presented. It takes part after the ring formation process and can last several hours depending on the size of the injury. 


\subsection{D motivation of actin-myosin contraction}

The general thermodynamic framework and the resulting constitutive equations for the actomyosin ring contraction are particularized for a 1D case in order to study the behavior towards different parameters and properties. At this stage, no coupling with the chemical part of the problem is considered, and a fully mechanical problem is solved. Considering a one dimensional pair of actin and myosin motors (see Figure 5.1 ), the thermodynamic framework is now adapted for the phenomenon of actomyosin contraction.

Figure 9: Actin-myosin pair. In order to produce the contraction of the cable, myosin motors pull together two different actin filaments towards itself. The result of this movement, when considering the macro scale and the interconnection of many of this mechanisms, is the shortening of the actomyosin cable and the contraction of the ring.

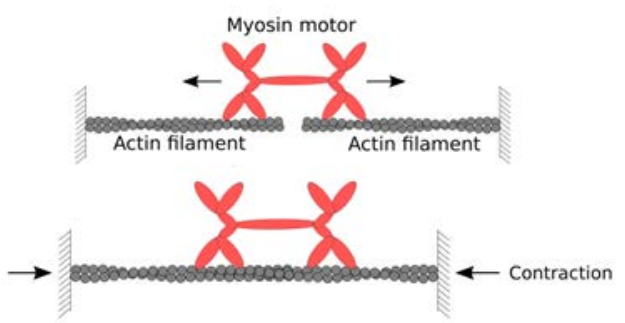

Together with the multiplicative decomposition of the deformation gradient, we here decompose in an additive manner the SEDF into a passive component $\Psi_{\mathrm{e}}$ and an active one $\Psi_{\mathrm{a}}[51]$,

$$
\Psi=\Psi_{\mathrm{e}}\left(\mathrm{J}, \mathrm{I}_{1}\right)+\Psi_{\mathrm{a}}\left(\lambda_{\mathrm{a}}, \lambda_{\mathrm{e}}\right) .
$$

where $\lambda_{a}, \lambda_{e}$ and $\lambda=\lambda_{e} \lambda_{a}$ are the projection of the active, elastic and total strain tensor into the direction of the fibers. In this $1 \mathrm{D}$ particularization, they correspond to the active, elastic and total stretches.

The second term, $\Psi_{\mathrm{a}}$, is the strain energy associated with the tissue's active response towards the actomyosin cable activity and can be expressed as a multiplication of two different functions

$$
\Psi_{\mathrm{a}}\left(\lambda_{\mathrm{a}}, \lambda_{\mathrm{e}}\right)=\mathrm{f}_{1}\left(\lambda_{\mathrm{a}}\right) \Psi_{\mathrm{a}}^{\prime}\left(\lambda_{\mathrm{e}}\right) .
$$

The first term $f_{1}\left(\lambda_{a}\right)$ is a scaling factor that accounts for the influence of the actin filament overlap, being $0<\mathrm{f}_{1}<1$. When there is insufficient or excessive filament overlap, the function becomes zero, resulting in null contraction of the actin cable. In the other hand, when the active stretch is near an optimum overlap, the function reaches its maximum. In order to reproduce this behavior the following Gaussian function is used

$$
\mathrm{f}_{1}\left(\lambda_{\mathrm{a}}\right)=\exp \left[-\frac{\left(\lambda_{\mathrm{a}}-\lambda_{\mathrm{opt}}\right)}{2 \xi^{2}}\right],
$$

being $\lambda_{\text {opt }}$ the optimal stretch at which the function reaches its maximum and $\xi$ a parameter that controls the slope of the function. Figure 10 depicts the different shapes when varying both parameters.

Figure 10: Actin filaments overlap $\mathrm{f}_{1}$ for different combinations of $\xi$ and $\lambda_{\text {opt }}$. Lower values for $\xi$ result on higher slopes, meaning that the overlap range where the contraction is possible is smaller.

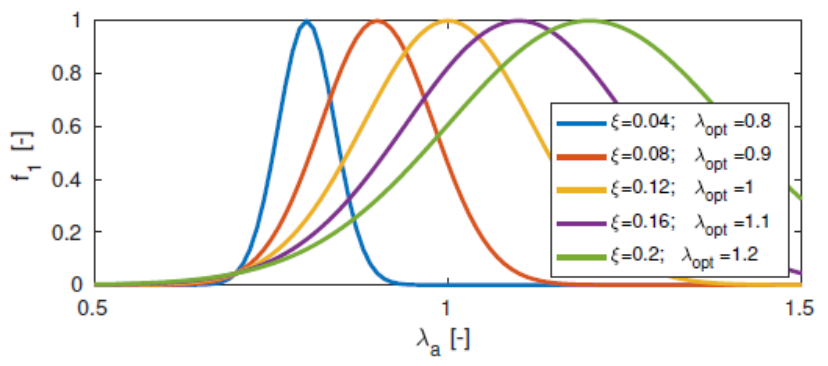

The second term in (44) is the free strain energy stored in the tissue due to the elastic deformation,

$$
\Psi_{a}^{\prime}=\frac{1}{2} P_{0}\left(\lambda_{e}^{2}-1\right)^{2}
$$


This function is dependent on the elastic strain and by a proportionality factor $P_{0}$, a factor related to the maximum stress caused by the contraction mechanism. The elastic stretch can be obtained from the multiplicative decomposition of the deformation gradient and therefore $\lambda_{e}=\lambda / \lambda_{a}$.

In order to evaluate (44), the active stretch $\lambda_{a}$ is needed [51], which is calculated explicitly via $\lambda_{\mathrm{a}}^{\mathrm{n}+1}=\lambda_{\mathrm{a}}^{\mathrm{n}}+\dot{\lambda}_{\mathrm{a}}^{\mathrm{n}} \Delta \mathrm{t}$ with

$$
\dot{\lambda}_{\mathrm{a}}=\mathrm{v}_{0}\left[-\mathrm{v}-\frac{1}{\mathrm{f}_{1}} \frac{\partial \mathrm{f}_{1}}{\partial \lambda_{\mathrm{a}}} \bar{\Psi}_{\mathrm{a}}+\frac{\lambda_{\mathrm{e}}}{\lambda_{\mathrm{a}}} \frac{\partial \bar{\Psi}_{\mathrm{a}}}{\partial \lambda_{\mathrm{e}}}\right],
$$

and where $\mathrm{v}_{0}$ is associated with the initial contraction velocity and $\mathrm{v}$ accounts for the friction due to the relative sliding speed between actin and myosin. Equation (47) is the 1D particularization of the general 3D contraction velocity. Its general deduction from the Clausius-Plank inequality can be found in Section 5.3.

\subsubsection{Numerical implementation}

In order to solve the $1 \mathrm{D}$ case with an uniform load $\mathrm{P}$, the minimization of the strain energy should be done for the total stretch, resulting on the equality,

$$
\sigma_{\mathrm{p}}+\sigma_{\mathrm{a}}+\mathrm{P}=0
$$

For this case only, instead of a Neo-Hookean model, the mechanical model proposed by Calvo et. al. [57] is implemented. Note that the volumetric component is ignored due to the fact that we have an unidimensional problem. The stresses obtained take the form

$$
\sigma_{\mathrm{p}}=\mu\left(\lambda^{2}-\frac{1}{\lambda}\right) \quad \text { and } \quad \sigma_{\mathrm{a}}=2 \mathrm{f}_{1} \lambda_{\mathrm{e}}^{2} \mathrm{P}_{0}\left(\lambda_{\mathrm{e}}-1\right)
$$

where (48) is solved iteratively through the Newton-Rapson method. In Table 1 the basic idea of the algorithm is listed.

\section{Algorithm}

1. Inital definitions:

External load. P

Material parameters and time-step size. $\lambda_{\text {opt }}, \xi, \mathrm{v}, \mathrm{v}_{0}, \mathrm{P}_{0}, \mu, \Delta \mathrm{t}$

Initial stretches. $\lambda, \lambda_{\mathrm{a}}$

2. $\operatorname{FOR}\left(\mathrm{t}_{0}: \Delta \mathrm{t}: \mathrm{t}_{\mathrm{f}}\right)$

Calculate $\dot{\lambda_{\mathrm{a}}}{ }^{\mathrm{n}}$ using $\lambda^{\mathrm{n}}$ and $\lambda_{\mathrm{a}}^{\mathrm{n}}$ using equation (47).

Calculate $\lambda_{\mathrm{a}}^{\mathrm{n}+1}=\lambda_{\mathrm{a}}^{\mathrm{n}}+{\dot{\lambda_{\mathrm{a}}}}^{\mathrm{n}} \Delta \mathrm{t}$ (explicit)

Calculate new $\mathrm{f}_{1}^{\mathrm{n}+1}$

Solve equilibrium $\sigma_{\mathrm{a}}+\sigma_{\mathrm{p}}=\mathrm{P}$ to find $\lambda^{\mathrm{n}+1}$ (N-R iterations)

END

Calculate $\lambda_{\mathrm{e}}^{\mathrm{n}+1}=\lambda^{\mathrm{n}+1} / \lambda_{\mathrm{a}}^{\mathrm{n}+1}$

Table 1: Algorithm for an implicit scheme of the active contraction of the actin ring.

\subsubsection{Parametric analysis}

The problem was solved for different combinations of material parameters with the aim of studying the influence of this constants on the behavior of the system while trying to achieve equilibrium. In all the cases, except when mentioned, an external load $\mathrm{P}$ was imposed gradually in the first stage of the simulation and kept constant for the rest of the time. 
The parameter $\xi$ which governs the sensitivity of the actomyosin ring towards filament overlap in equation (45) was set to different values. As a result the region in which $\mathrm{f}_{1}$ is different from zero changed, resulting on different evolution of the overlap factor over time. In Figure 11 this evolution can be observed, along the active and total stretch. Larger values of $\xi$ result in faster retrieving of the initial stretch and lower stable active contractions. For small enough values of $\xi$ the original stretch is not retrieved, which can be explained by the fact that the overlap window is too small and the resultant active contraction is unable to pull the tissue back to the original stretch.

Now, the proportionality factor $\mathrm{P}_{0}$ that affects the scale of equation (46) was set to different values. In Figure $11 \mathrm{~b}$ the different outcomes can be observed. The larger the value for $\mathrm{P}_{0}$, the faster the initial stretch is retrieved and the slower the stable state for $f_{1}$ is achieved. Note that, for a null proportionality factor, although $\lambda_{a}$ evolves due to the active part of the minimization problem, the active stress is always zero and the total stretch does not move from its value once the loading stage has ended.

The initial contraction velocity $\mathrm{v}_{0}$ that scales equation (47) was set to different values. Note that different signs were used to generate an active contraction or expansion as shown in Figure 11c. For positive values of $\mathrm{v}_{0}$, there is a positive active stretch or expansion, increasing with it the total stretch. For negative values, a negative active stretch or contraction occurs, resulting on the retrieving of the initial stretch. Note that for a null value, no further evolution happens once the loading stage has ended.

Finally, the friction parameter v, which introduces an evolution of the active stretch in (47) independently of the external load, was set to different values. Note that for this case, and for a better observation of the mentioned characteristic, the external load is set to zero, i.e. $\mathrm{P}=0$. The results for the different tested cases can be appreciated in Figure 11d. For $\mathrm{v}=0$ no evolution of any function occurs, but when $\mathrm{v}>0$ there is a natural evolution of the active stretch independently of the external load. We note that the behavior of the stretches during the loading stage is practically identical for all choices of the parameters. Also, for large enough values of $\mathrm{v}$, the resulting stretch can be highly influential on the evolution of all of the curves.

\subsection{Contraction velocity}

Assuming that the active contraction occurs in a given direction $\mathbf{m}_{0}$, a local active deformation tensor can be defined, in this case for a $2 \mathrm{D}$ framework

$$
\mathbf{F}_{\mathrm{a}}^{\prime}=\lambda_{\mathrm{a}} \mathbf{M}+\lambda_{\mathrm{a}}^{-1 / 2} \mathbf{N} \quad \text { with } \quad \mathbf{M}=\mathbf{m}_{0} \otimes \mathbf{m}_{0} \quad \text { and } \quad \mathbf{N}=\mathbf{n}_{0} \otimes \mathbf{n}_{0},
$$

where $\lambda_{a}$ is the active stretch. The two structural tensors $\mathbf{M}$ and $\mathbf{N}$, defined in the reference configuration, depend on the anisotropic directionality of the actin fibers distribution, given by the unit vector $\mathbf{m}_{0}$ and its perpendicular direction such that $\mathbf{m}_{0} \cdot \mathbf{n}_{0}=0$.

To obtain the contraction velocity $\dot{\lambda}_{\mathrm{a}}$ explicitly, the time derivative of the active deformation tensor is obtained, using the chain rule

$$
\dot{\mathbf{F}}_{\mathrm{a}}=\frac{\partial \mathbf{F}_{\mathrm{a}}}{\partial \lambda_{\mathrm{a}}} \dot{\lambda}_{\mathrm{a}} \quad \text { where } \quad \frac{\partial \mathbf{F}_{\mathrm{a}}}{\partial \lambda_{\mathrm{a}}}=\mathbf{R}^{\mathrm{T}} \mathbf{M R}-\frac{1}{2} \lambda_{\mathrm{a}}^{-3 / 2} \mathbf{R}^{\mathrm{T}} \mathbf{N R} .
$$

with $\mathbf{R}$ the matrix that rotates $\mathbf{m}_{0}$ onto its spatial orientation, and $\mathbf{F}_{a}=\mathbf{R}^{t} \mathbf{F}_{a}^{\prime} \mathbf{R}$. Note that we have assumed that directions $\mathrm{m}_{0}$ and $\mathrm{n}_{0}$, and rotation matrix $\mathrm{R}$ are all constant. Considering that the contraction will only occur along acto-myosin element we can write the Clausius-Plank inequality as

$$
\left[\mathrm{P}_{\mathrm{a}}-\frac{\partial \Psi}{\partial \lambda_{\mathrm{a}}}+\left(2 \mathbf{C}_{\mathrm{e}} \frac{\partial \Psi}{\partial \mathbf{C}_{\mathrm{e}}} \mathbf{F}_{\mathrm{a}}^{-\mathrm{T}}\right): \frac{\partial \mathbf{F}_{\mathrm{a}}}{\partial \lambda_{\mathrm{a}}}\right] \dot{\lambda}_{\mathrm{a}} \geq 0
$$

which leads to

$$
\mathrm{P}_{\mathrm{a}}-\frac{\partial \Psi}{\partial \lambda_{\mathrm{a}}}+\left(2 \mathbf{C}_{\mathrm{e}} \frac{\partial \Psi}{\partial \mathbf{C}_{\mathrm{e}}} \mathbf{F}_{\mathrm{a}}^{-\mathrm{T}}\right): \frac{\partial \mathbf{F}_{\mathrm{a}}}{\partial \lambda_{\mathrm{a}}}=\mathrm{c} \dot{\lambda}_{\mathrm{a}}
$$



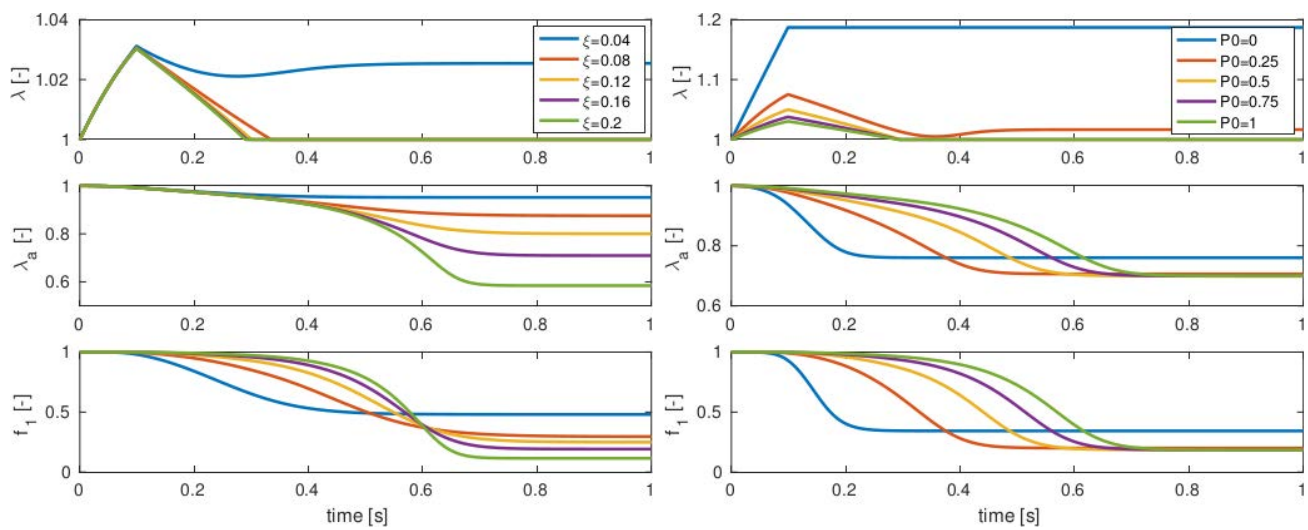

(a)

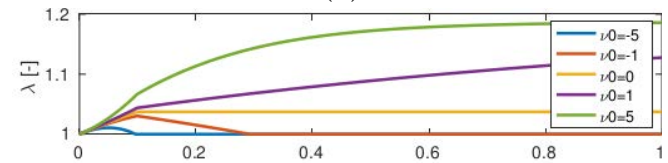

(b)
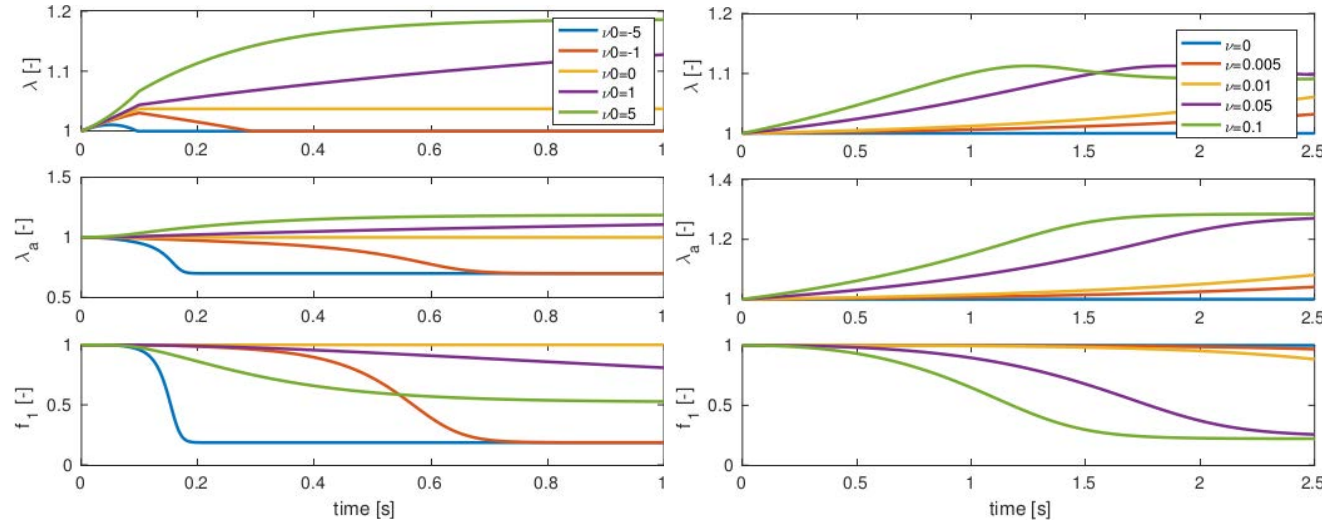

(c)
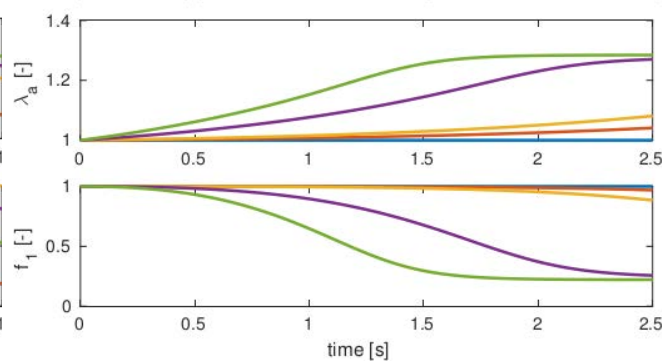

(d)

Figure 11: Evolution of total stretch $\lambda$, active stretch $\lambda_{\mathrm{a}}$ and overlap influence parameter $\mathrm{f}_{1}$ for different values of (a) $\xi$, (b) $\mathrm{P}_{0},(\mathrm{c}) \mathrm{v}_{0}$ and $(\mathrm{d}) \mathrm{v}$ (see equations (45) and (47)).

with $\mathrm{c}=\mathrm{c}\left(\lambda_{\mathrm{a}}\right) \geq 0$. In order to obtain the contraction velocity of the actomyosin cable, two components are defined such that (53) is satisfied

$$
\mathrm{c}=\frac{1}{\mathrm{v}_{0}\left(\rho^{*}\right)} \mathrm{P}_{0} \mathrm{f}_{1}\left(\lambda_{\mathrm{a}}\right) \quad \text { and } \quad \mathrm{P}_{\mathrm{a}}=-\mathrm{vP}_{0} \mathrm{f}_{1}\left(\lambda_{\mathrm{a}}\right)
$$

Replacing both equations in (54) and the definition of the active SEDF (44) into (53), and considering the definition of the fourth elastic invariant $\mathrm{I}_{4}^{\mathrm{e}}=\mathbf{m}_{0} \cdot \mathbf{C}_{\mathrm{e}} \mathbf{m}_{0}$, the general expression for the velocity of the active contraction is obtained

$$
\dot{\lambda}_{\mathrm{a}}=\mathrm{v}_{0}\left(\rho^{*}\right)\left[-\mathrm{v}-\frac{1}{\mathrm{f}_{1}} \frac{\partial \mathrm{f}_{1}}{\partial \lambda_{\mathrm{a}}} \Psi_{\mathrm{a}}^{\prime}+2 \frac{\lambda_{\mathrm{e}}^{2}}{\lambda_{\mathrm{a}}} \frac{\partial \Psi_{\mathrm{a}}^{\prime}}{\partial \mathrm{I}_{4}^{\mathrm{e}}}\right]
$$

The coupling with the actin concentration distribution, takes place through the active contraction velocity, where the velocity $\mathrm{v}_{0}$, which scales (55), is defined as a function instead of a constant

$$
\mathrm{v}_{0}\left(\rho^{*}\right)=\frac{\mathrm{v}_{0}^{\max }}{1+\mathrm{e}^{-\kappa\left(\rho^{*}-\zeta\right)}} \quad \text { with } \quad \rho^{*}=\rho / \rho_{0},
$$

where $\mathrm{v}_{0}^{\max }$ is the maximum possible value for the velocity, $\zeta$ is a parameter that determines a trigger value of relative actin concentration at which there exists active contraction and $\kappa$ controls smoothness of the actin cable region. The different velocity responses towards different actin concentrations as this parameters change can be observed in Figure 12. 
Figure 12: Initial contraction velocity $v_{0}$ for different parameters of $\kappa$ and $\zeta$ as a function of the relative actin concentration $\rho / \rho_{0}$. The higher the value for $\kappa$, the bigger the slope of the curve and more defined is the area at which the active contraction occurs. Parameter $\zeta$ sets the position of the curve, defining the trigger value of $\rho^{*}$ at which there exist an update in the active stretch.
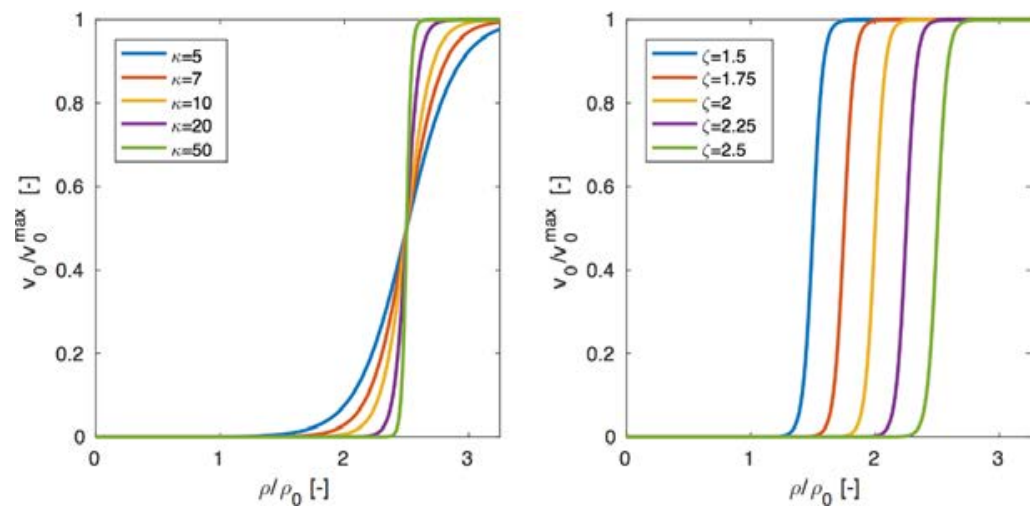

Remark 2 (FEM implementation). The equations presented here are solved in the finite element framework given in Section 3, and its linearized form in 3.4. Note that as the diffusion problem is already in steadystate situation from the previous actin ring formation, only the mechanical part is solved, and therefore the problem reduces to,

$$
\mathbf{R}_{i(n+1)}^{\varphi(k+1)}=\mathbf{R}_{i(n+1)}^{\varphi(k)}+\mathrm{d} \mathbf{R}_{i}^{\varphi}=0
$$

The active stretch update is done explicitly at each time-step, using the the expression for $\dot{\lambda}_{\mathrm{a}}$ given in $(55)$.

\subsection{Constitutive equation of the contraction mechanics}

In the previous section, the mechanics through which the actin cable contracts were explained. Now, the general constitutive equations and their derivation from the kinematic framework are developed. Additionally, the coupling with the actin concentration obtained in the previous section is explained.

The process of contraction of the actomyosin ring starts around 15 minutes after the wound is inflicted, when the actin concentration stabilizes around the wound margin and the myosin motors start to work in a coordinated manner. In order to model the phenomenon in our finite element framework, we perform the kinematic decomposition of the deformation gradient, as shown in equation (2), where now the arbitrary deformation gradient $\mathbf{F}_{(\cdot)}$ takes the form of the active tensor $\mathbf{F}_{a}$ specified in the previous section.

Along with this kinematic description of the problem, we can reformulate the SEDF, again following the same reasoning as in Section 4 , by adding both elastic energy $\Psi_{e}$ and the active energy $\Psi_{a}$ as

$$
\Psi=\Psi_{e}\left(\mathbf{C}_{e}\right)+\Psi_{a}\left(\mathbf{C}_{e}, \mathbf{F}_{a}\right) .
$$

The equations for the non-linear elastic part can be obtained through the definition of a strain energy density function $\Psi_{e}\left(\mathbf{C}_{e}\right)$ as in (14). The first term of the Clausius-Plank inequality is now decoupled, and the elastic and active contributions can be identified

$$
\mathcal{D}_{\text {int }}=\frac{1}{2} \mathbf{S}: \dot{\mathbf{C}}+\frac{1}{2} \mathbf{S}_{a}: \dot{\mathbf{C}}_{a}-\dot{\Psi} \geq 0 .
$$

Replacing equation (58) into equation (59) in its material form, the following expression is obtained

$$
\left[\frac{1}{2} \mathbf{S}-\frac{\partial \Psi}{\partial \mathbf{C}}\right]: \dot{\mathbf{C}}-\frac{\partial \Psi}{\partial \mathbf{C}_{e}}: \dot{\mathbf{C}}_{e}+\left[\frac{1}{2} \mathbf{S}_{a}-\frac{\partial \Psi}{\partial \mathbf{C}_{a}}\right]: \dot{\mathbf{C}}_{a} \geq 0
$$

and making use of equation (13) the inequality can be rewritten as

$$
\left[\frac{1}{2} \mathbf{S}-\frac{\partial \Psi}{\partial \mathbf{C}}-\mathbf{F}_{a}^{-1} \frac{\partial \Psi}{\partial \mathbf{C}_{e}} \mathbf{F}_{a}^{-T}\right]: \dot{\mathbf{C}}+\left[\mathbf{F}_{a} \mathbf{S}_{a}-2 \mathbf{F}_{a} \frac{\partial \Psi}{\partial \mathbf{C}_{a}}+2 \mathbf{C}_{e} \frac{\partial \Psi}{\partial \mathbf{C}_{e}}: \dot{\mathbf{C}}_{e} \mathbf{F}_{a}^{-t}\right]: \mathbf{F}_{a} \geq 0
$$


from which two constitutive equations can be obtained:

$$
\begin{aligned}
& \mathbf{S}=2 \frac{\partial \Psi}{\partial \mathbf{C}}+\mathbf{F}_{a}^{-1} \frac{\partial \Psi}{\partial \mathbf{C}_{e}} \mathbf{F}_{a}^{-t} \\
& {\left[\mathbf{F}_{a} \mathbf{P}_{a}-2 \mathbf{F}_{a} \frac{\partial \Psi}{\partial \mathbf{C}_{a}}+2 \mathbf{C}_{e} \frac{\partial \Psi}{\partial \mathbf{C}_{e}} \mathbf{F}_{a}^{-t}\right]: \mathbf{F}_{a} \geq 0 .}
\end{aligned}
$$

Finally, considering the additive decomposition of the SEDF in (58), the second Piola-Kirchoff stress can also be considered as an additive expression

$$
\mathbf{S}=2 \frac{\partial \Psi}{\partial \mathbf{C}}=2 \frac{\partial \Psi_{e}}{\partial \mathbf{C}}+2 \frac{\partial \Psi_{a}}{\partial \mathbf{C}}=\mathbf{S}_{e}+\mathbf{S}_{a}
$$

Here, the elastic contribution is the same as the one defined in equation (14). Regarding the active part of the SEDF, the generalized Hill model, used by [58] to simulate muscle contraction is used, as the micro-scale mechanism of the actin-myosin interaction is basically the same. The SEDF reads $\Psi=\Psi_{\mathrm{e}}\left(\mathrm{J}, \mathrm{I}_{1}\right)+\Psi_{\mathrm{a}}\left(\lambda_{\mathrm{a}}, \lambda_{\mathrm{e}}\right)$ where the active component of the energy is $\Psi_{\mathrm{a}}\left(\mathrm{I}_{4}^{\mathrm{e}}\right)=0.5 \mathrm{P}_{0}\left(\mathrm{I}_{4}^{\mathrm{e}}-1\right)^{2}$, where the elastic invariant used accounts for the active part of the deformation is $\mathrm{I}_{4}^{\mathrm{e}}=\mathbf{m}_{0} \cdot \mathbf{C}_{\mathrm{e}} \mathbf{m}_{0}=\lambda_{\mathrm{e}}^{2}$.

In order to solve the problem, analogously to Section 4, we need to compute the derivatives of $\mathbf{S}_{e}$ and $\mathbf{S}_{a}$ with respect to the Cauchy-Green deformation tensor $\mathbf{C}$. Their deduction is given in constitutive operator expression are needed. To do so, each part of the SEDF is derived with respect to the Cauchy-Green deformation tensor, knowing that $\mathbf{S}=\mathbf{S}_{\mathrm{e}}+\mathbf{S}_{\mathrm{a}}$. As for the elastic part

$$
\mathbf{S}_{\mathrm{e}}=2 \partial_{\mathbf{C}} \Psi_{\mathrm{e}}^{\text {iso }}+2 \partial_{\mathbf{C}} \Psi_{\mathrm{e}}^{\mathrm{ani}}=(\lambda \ln (\mathrm{J})-\mu) \mathbf{C}^{-1}+\mu \mathbf{I} .
$$

We define the structural tensor that accounts for the anisotropy of the line elements, constructed with by the direction of the actin fibers, is $\mathbf{M}=\mathbf{m}_{0} \otimes \mathbf{m}_{0}$. The active contribution to the stress tensor is obtained by pulling back the derivative of the SEDF with respect to the elastic Cauchy-Green deformation tensor

$$
\mathbf{S}_{a}=2 \mathbf{F}_{a}^{-1}\left[\hat{\mathbf{S}}_{a}\right] \mathbf{F}_{a}^{-t} \quad \text { with } \quad \hat{\mathbf{S}}_{a}=\partial_{\mathbf{C}_{e}} \Psi=2 P_{0}\left(I_{4}^{e}-1\right) \mathbf{M}
$$

Analogously to Section 4, and in order to use the Newton-Raphson iterative process, we need to compute the derivatives of $\mathbf{S}_{e}$ and $\mathbf{S}_{a}$ with respect to the Cauchy-Green deformation tensor $\mathbf{C}$. Tensor $\mathbb{C}_{e}=2 \mathrm{~d}_{\mathbf{C}} \mathbf{S}_{e}$, similar to the one described in 4.4, and $\mathbb{C}_{a}=2 \mathrm{~d}_{\mathbf{C}} \mathbf{S}_{a}$, sum up to form the total elastic modulus $\mathbb{C}=\mathbb{C}_{e}+\mathbb{C}_{a}$. The active part is simply defined as the pull back of the term $\mathbb{C}_{a}$ with the active deformation gradient as

$$
\mathbb{C}_{a}=\left[\mathbf{F}_{a}^{-1} \bar{\otimes} \mathbf{F}_{a}^{-1}\right]: \hat{\mathbb{C}}_{a}:\left[\mathbf{F}_{a}^{-t} \bar{\otimes} \mathbf{F}_{a}^{-t}\right] \quad \text { with } \quad \hat{\mathbb{C}}_{a}=4 \partial_{\overline{\mathbf{C}}_{e}}^{2} \Psi_{a}=4 P_{0} \mathbf{M} \otimes \mathbf{M}
$$

The material elastic moduli $\mathbb{A}=\mathrm{d}_{\mathbf{F}} \mathbf{P}_{e}$, necessary for computing $\mathbb{C}_{e}$, is obtained using relationship (30).

\section{Results}

In this section, some numerical examples of the previously described models are presented and their comparison with experimental results are discussed. In Section 6.1 the wound geometry and finite element meshes are described along with their biological counterpart. In Section 6.2 the initial wound expansion for the different geometries is performed, showing the mechanical consequences of the wound infliction over a pre-strained epithelium sheet. In Section 6.3, using the results from previous section, the ring formation stage is presented. In Section 6.2 and 6.3, the model described in Section 4 is used. The different constants were fitted through different test cases. In Section 6.4 the wound closure through the actomyosin ring contraction is studied, using the model described in Section 5 along with the results obtained in Section 6.3 as initial conditions. We present in Table 2 a summary of the mechanisms analyzed as a function of the timing, the balance and the constitutive equation involved and coupling procedure between them. 


\begin{tabular}{|l|l|l|l|}
\cline { 3 - 4 } \multicolumn{2}{c|}{} & Strong Form & Constitutive Eq. \\
\hline \multirow{2}{*}{ Ring formation } & 1. Calcium firing and transport & $\dot{\phi}-\nabla \cdot Q=F^{\phi}\left(I_{1}, \phi\right)$ & Eq. 33, 34, 35, 36 \\
\cline { 2 - 4 } & 2. Actin formation & $\dot{\rho}=F^{\rho}(\phi)$ & Eq. 38, 14 \\
\cline { 2 - 4 } & 3. Mechanical response & $-\nabla \cdot P\left(\mathbf{F}_{e}, \mathbf{F}_{g}\right)=0$ & Eq. 39, 40 \\
\hline Ring contraction & 4. Active contraction of actin $\mathrm{ring}$ & $-\nabla \cdot P\left(\mathbf{F}_{e}, \mathbf{F}_{a}\right)=0$ & Eq. 54, 55, 56 \\
\hline
\end{tabular}

Table 2: Description of the main mechanisms during the actin ring formation (short time) and the active contraction of the actin ring at the long time. Table summarises the strong form of the equations that governs each mechanisms as well as the constitutive equation involved. The calcium firing and transport is fully coupled with the mechanics of the tissue (mechanisms 1 and 3) and it is solved implicitly. The actin ring formation and the consequent acto-myosin contraction (mechanism 2) is solve explicitly as a function of the calcium wave (mechanism 1). The constitutive relation that govern the active contraction of the actin ring (mechanism 4), once the ring is formed, is solved implicitly in a N-R iteration.

\subsection{Wound geometry and FEM meshes}

With the objective of comparing the numerical and experimental results, the shape of the wound used for the numerical cases is inspired by those ones found in the biography [5]. The shape of the injure was set to be a circular domain with an external diameter equal to $400 \mu \mathrm{m}$. This corresponds to the diameter of the laser ablation. Regarding the finite element discretization, 5760 linear quadrilateral elements were used, with increase density towards the wound, to account for the high gradients obtained when deforming the mesh. Note that the mesh is structured, with 40 elements in the radial direction and 144 elements in the tangential direction. A numerical integration with two Gauss points along each direction is used.

\subsection{Wound expansion}

In this section, the stresses obtained through the imposition of a pre-strain field to the initial mesh are presented. The idea behind it is to simulate the wound expansion produced when a laser ablates the tissue. Firstly, different combinations of pre-strains were tested for different Young modulus in order to evaluate the stresses for different imposed deformations. We aimed to investigate the effect of these two material parameters that have been presented in the literature with some variability. Stress profiles were clearly scaled between each other with the elastic modulus and pre-strains, although the increase of the wound diameter behaved slightly nonlinearly with respect to the pre-strains variations. In Figure 13, the resulting tension map for an elastic modulus of $\mathrm{E}=0.5 \mathrm{kPa}$ and different combinations of pre-strains can be observed. The experiment was repeated for different values of the Young modulus $\mathrm{E}=1.0 \mathrm{kPa}$ and $\mathrm{E}=10 \mathrm{kPa}$, and the same Poisson coefficient $\nu=0.45$. These two values resulted in the same distribution pattern, but scaled by a 2 - and a 20 -fold increase due to the relatively low non-linearity of the model adopted here. Note that for the three prescribed deformation tensors, different wound diameter increase can be observed, being $34 \%, 69 \%$ and $115 \%$ bigger than the original opening for $\mathrm{f}_{\text {pre }}=0.97, \mathrm{f}_{\text {pre }}=0.95$ and $\mathrm{f}_{\text {pre }}=0.93$ respectively (see equation (31)).

Figure 13: Wound expansion. Stresses and deformation due to imposition of a prescribed deformation tensor $\mathbf{F}_{0}$ for circular wound for a epithelium described with a Young modulus $\mathrm{E}=0.5 \mathrm{kPa}$. For three different scalar stretch factors $\mathrm{f}_{\text {pre }}$, see equation (31), the resulting stresses in the principal directions, $\sigma_{11}$ (circumferential) and $\sigma_{22}$ (radial), are plotted.
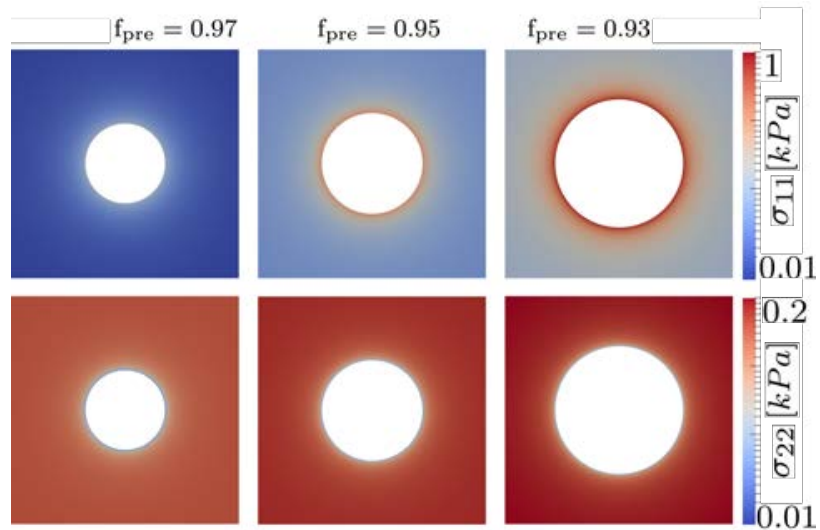


\subsection{Actomyosin ring formation}

In this section, the results for the actin ring formation are presented. To calculate them, the wound expansion results for $\mathrm{E}=1 \mathrm{kPa}$ and $\mathrm{f}_{0}=0.95$ were used [22]. The results for 60 seconds of the fully coupled problem are shown in figures 13 and 14 at 3 time-steps. The obtained results are for the combination of coupling parameters from Table 3. The formation of an actin ring can be clearly noticed. Calcium is created in the wound boundaries, diffusing away from the gap junction. When the calcium reaches the necessary concentration, in a specific concentration interval, actin is created. The actin does not diffuse but the mentioned calcium wave moves away from the wound, moving the actin recruitment region away from the injury edge.

Although these in silico results did not reproduce exactly the ones observed in [5] at long times, they did reproduce the calcium evolution at shorter time scale $[8,9]$. When the acto-myosin concentration reaches a given value, cells react and contract, which can be observed in the simulated contraction parameter, $\vartheta$, stress and deformation maps in Figure 14. As it can be observed in Figure 14(A), relative calcium concentration increases due to calcium source term. As time passes, the increase of calcium in the wound border slows down, while the limit concentration is being reached (see Figure 14(B)). The profile of the relative actin concentration can be noted to grow, due to the moving actin source term. The material suffers deformations, as slightly noticeable in Figure 14(E) and highly in (F) along with the corresponding distribution of actin and growth multiplier in (I). Concurrent increase of stresses in both principal directions can be also observed in Figure 14(F)-(G).
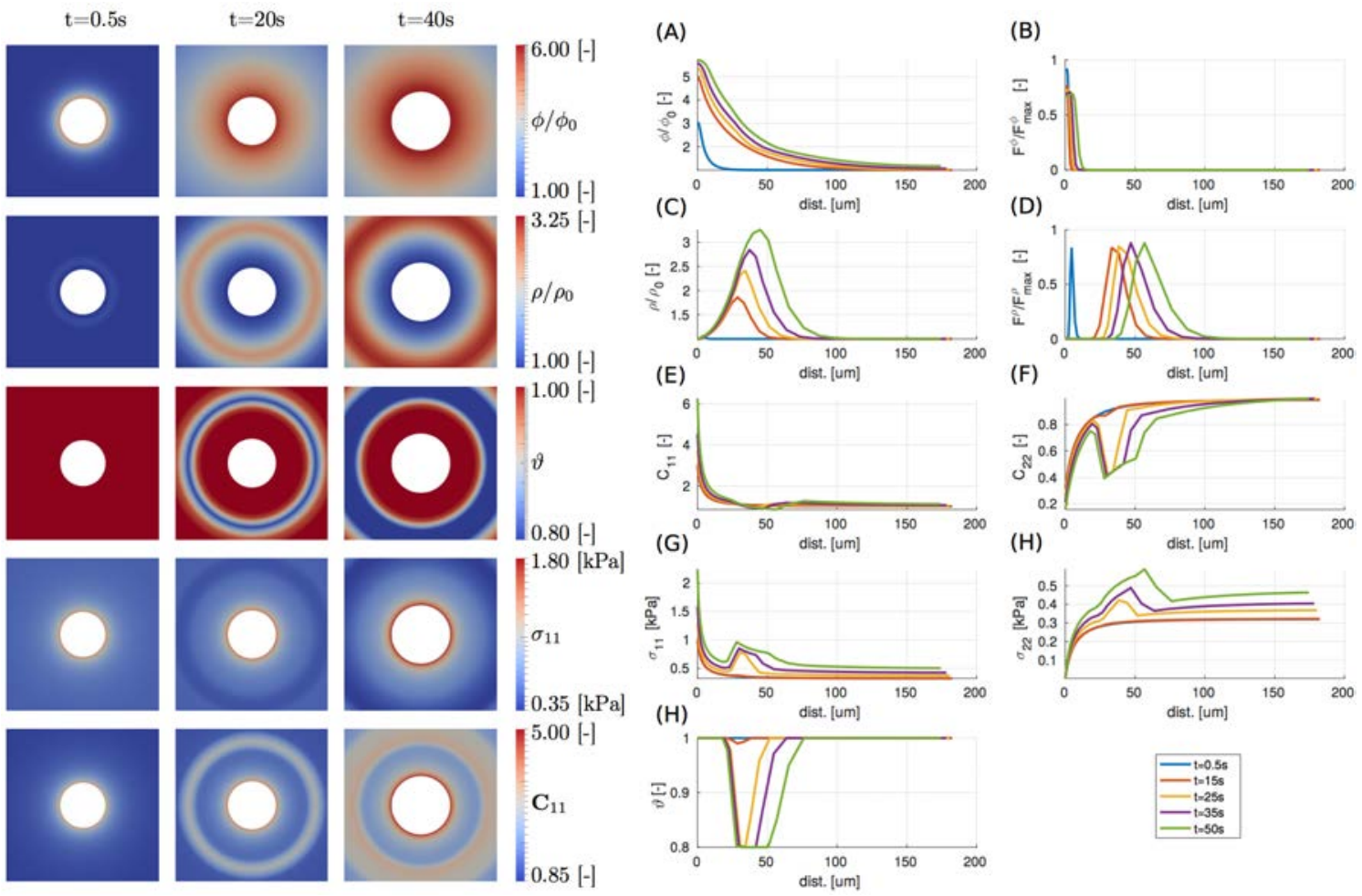

Figure 14: Left: Evolution of relative concentration of calcium $\left(\phi / \phi_{0}\right)$, actin $\left(\rho / \rho_{0}\right)$, growth multiplier $(\vartheta)$, maximum principal stress $\left(\sigma_{11}\right)$ and Cauchy-Green deformation gradient in the first principal direction $\left(\mathbf{C}_{11}\right)$ for three different time steps. First principal direction is circumferential. Right: Plot over a line that gets away from the wound in a radial direction, through different time steps. (A) Relative calcium concentration. (B) Relative calcium source term. (C) Relative actin concentration. (D) Relative action source term. (E)-(H) Principal components of Cauchy-Green strain tensor and Cauchy stress tensor in circumferential and radial directions. (I) Distribution of growth multiplier $\vartheta$. 


\begin{tabular}{ccccc}
\hline \multicolumn{5}{c}{ Coupling parameters } \\
\hline$\nu=100 \mu m^{2} / s$ & $\gamma=2.5$ & $\delta=0.2$ & $\vartheta_{\min }=0.8$ & $\omega=2$ \\
$\beta=25$ & $\phi *$ lim $=10$ & $\epsilon=2$ & $\tau=25$ & $\rho *_{l i m}=3.25$ \\
\hline \multicolumn{4}{c}{ Table 3: Parameters used for the calculations in Figure 14. }
\end{tabular}

In order to study the behavior of the model with different values, several circular wound cases were run with different combinations. These results are included in the Appendix. To generate an actin ring around the wound, parameter $\epsilon$ in equation (38) was chosen to be equal to the maximum calcium concentration, and therefore create actin around the wound. In Figure 15 the results for simulations of 7.5 minutes long are shown, using the parameters in Table 3 and $\omega=5$ (see equation (40)). The actin wave was not achieved as described by [5] but similar to what have been reported in other epithelial systems [59, 8, 9]. It can be noted that with the advance of time, calcium is created in the wound boundaries, diffusing away from the gap, evolving towards an uniform concentration. When the calcium reaches the necessary concentration, the final actin region around the wound edge needed for the ring contraction state was successfully obtained. Although not shown explicitly in the pictures, when the actin concentration reaches a given value, the tissue reacts and contracts, as it can be observed in the stress and deformation maps of Figure 15.

Figure 15: Actomyosin ring formation of a stable table ring. Initial and final distribution of relative concentration of calcium $\left(\phi / \phi_{0}\right)$, relative actin $\left(\rho / \rho_{0}\right)$, maximum principal Cauchy stress, $\sigma_{11}$, and Cauchy-Green deformation gradient, $\mathbf{C}_{11}$, in the first principal direction (circumferential) in the ring formation stage.
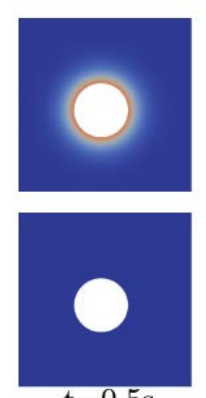

$\mathrm{t}=0.5 \mathrm{~s}$
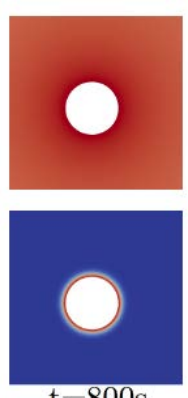

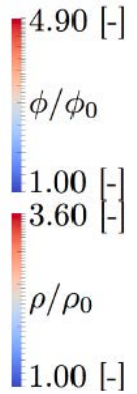

$\mathrm{t}=800 \mathrm{~s}$
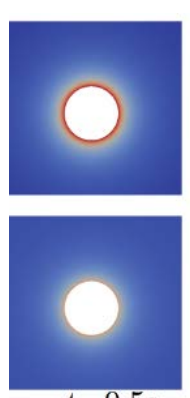
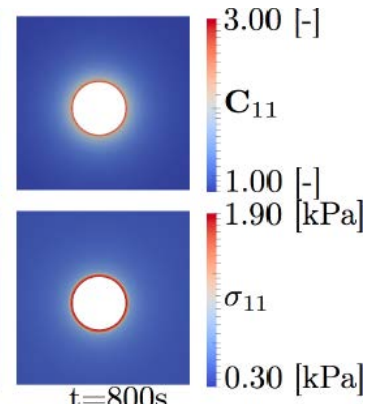

$3.00[-]$
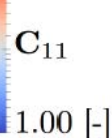

$\mathrm{t}=800 \mathrm{~s}$ $\mathrm{t}=0.5 \mathrm{~s}$

\subsection{Ring contraction stage}

In this section, the results for the active actomyosin ring contraction, based on the model from Section 5 are presented. To calculate them, the results from Section 6.3 were used as initial conditions, particularly those which presented a stable distribution of actin, forming a ring around the wound edge, such as in Figure 15. To perform the simulation, parameters $\kappa$ and $\zeta$ from equation (56) were chosen to create a region of active contraction (actomyosin ring) from the continuous distribution of actin along all the domain.

First, the results obtained during the contraction stage of the wound are shown in Figure 16, for the parameters shown in Table 4. Along with a diameter reduction of a 55\%, all the mechanical parameters, principal strains $\mathbf{C}_{11}$ and $\mathbf{C}_{22}$ and principal stresses $\sigma_{11}$ and $\sigma_{22}$, tend to become more uniform as the active deformation compensates the previous ones.

\begin{tabular}{ccccccc}
\hline \multicolumn{8}{c}{ Contraction parameters } \\
\hline $\mathrm{v}_{0 \max }=-1$ & $\mathrm{v}=0$ & $\mathrm{P}_{0}=1 k P a$ & $\lambda_{\text {opt }}=0.4$ & $\xi=0.12$ & $\kappa=0.25$ & $\zeta=2.25$ \\
\hline
\end{tabular}

Table 4: Parameters used for the calculations of Figure 16.

For the same simulation as in Figure 16, a point in the wound edge is tracked over time to study the velocity of the area reduction, which can be observed in Figure 17. The computational results are compared with experimental ones facilitated by Yanlan Mao's research group. The results predict a fast closing in the first 5 minutes after wound recoiling, very similarly to the closing rates observed in the experimental results. After 10 minutes, the numerical results diverge from the experiments, describing a plateau in which 

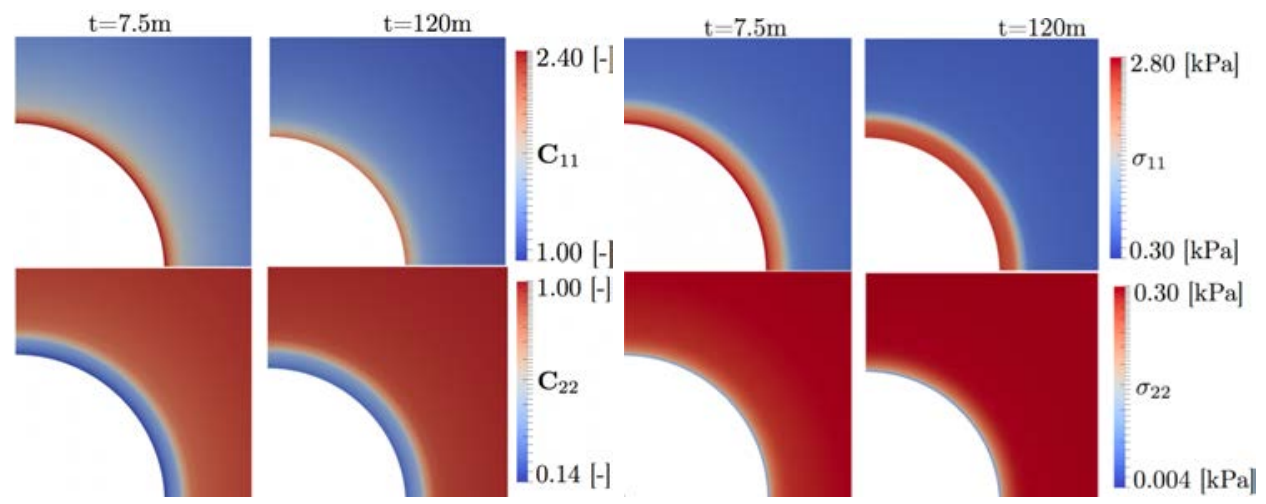

Figure 16: Actomyosin ring contraction. Left: strains before and after active contraction. Right: stresses before and after contraction.

the closure stops while in reality wound progress up to an almost complete closure. This could be due to the effect of cell crawling which is responsible for the total closure of the wound. These effects have not been included in the model. However, it is believed that cell crawling would have little affect on the apical dynamics in this particular biological system. Other cell behaviours could not be captured in the model.

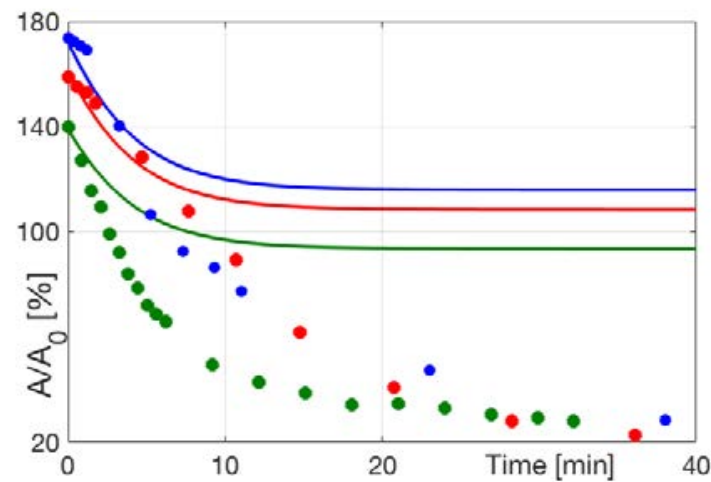

Figure 17: Actomyosin ring contraction. Evolution of wound area. The contraction velocity decreases from $0.84 \mu \mathrm{m}^{2} / \mathrm{min}$ at the beginning of the contraction stage to practically zero as the wound edge advances. As a result, the original wound area is never retrieved and the wound does not fully close. Solid lines represent the results of the computational model. Solid circles are experimental results facilitated by Yanlan Mao's research group.

\section{Discussion}

The transcendence of wound healing to biology has long been highlighted and its role in medicine demonstrated. In consequence, wound healing has been the focus of research for years. The closure of gaps is key for epithelium integrity during developmental and wound healing. Physical forces arise through the assembly of actomyposin-based contractile purse strings and/or cell crawling. Experimental results have been extensive during the last decades (see $[60,6]$ ).

The process of the formation of the actomyosin ring consists on simultaneous action of different phenomena. The mechano-sensitive pathway, e.g the opening of strain activated ion channels [61], as a response to residual strains from the wound expansion stage, induce a firing of calcium either extra- or intra-cellularly that propagates through the epithelium $[21,61,11]$. An adequate amount of intracellular calcium triggers the recruitment of actin filaments and myosin motors through the action of Rho GTPases [62], which promotes a short time contraction of the cells. Through the coupled interaction of this three mechanisms, the actomyosin recruitment grows and migrates towards the wound edge. Once in position, the myosin motors act upon the actin fibers, producing an active contraction of the ring which drives the gap edges together. Next, cell crawling may acts simultaneously to end up knitting the wound. Apart from remarkable advance from experimental biology, the theoretical analysis of the physical forces that acts in the wounded region has been much less explored. In this work, we make use of a coupled computational continuum model in order to address the complex interplay of chemical transport and mechanics in the still 
widely unknown problem of wound healing in epithelia.

First, we model the coupling of the calcium firing and transport that eventually promotes the actin recruitment and actomyosin ring formation. We managed to reproduce the deformation state in the wound periphery, reported by [5]. The tissue stretches in the direction of the wound edge while it compresses in the perpendicular direction. Further experimental measurements could confirm further deformation and stress measures.

Our simulations reproduce closely the calcium profiles as well as the actin patterns within the wounded region founded at short time in experimental observations $[8,9]$. In the numerical solution (Figure 14(A)), and also in the experimental results, we found that a ring-kind profile in the calcium concentration, which is maximum at the wound edge and decreases outwards and along time. However, at long times, the numerical cases performed with the developed model could not reproduce the experiments from Antunes et. al. [5]. In the experimental case, Figure 3, the profile has a minimum around the wound edge and a maximum that starts distant from it and advances forwards, loosing intensity. We were not able to rationalize the complex dynamics of the calcium profiles, which it is still an open question in experimental biology. Further experimental and theoretical work could improve our understanding on how mechanics and calcium dynamics are orchestrated in wound healing.

In terms of the acto-myosin profile, we have been able to reproduce very distinctive aspects of its spatial and time evolution. At the transient state, Figure 4, the actin distribution has a stable concentration around the wound edge. Far away from it advances toward the wound edge until an stable actin purse-string is form at the wound contour. By defining the parameter $\epsilon$ in the numerical case, which controls the calcium concentration at which the actin source term reaches its maximum, the actin ring is created away from the wound edge, but the evolution of the calcium profile provokes its migration in the opposite direction than the experimental case, as seen in Figure 14. In this aspect, the numerical simulation was not able to reproduce the experimental results reported by Antunes et. al. [5]. This is directly related with the discrepancies in the calcium wave describe above. Further experimental and theoretical work will be required to answer this question. However, we were able to resolve the transient wave contraction that propagates in the epithelium. In fact, the inherent orchestration of the acto-myosin network reduces back the deformation of the peripheral cells and consequently of the calcium source. Finally, by the manipulation of the model parameters, the ring is formed and stays around the wound as described in Figure 15 and reported in a number of experimental observations described along this manuscript.

To asses the actomyosin ring contraction stage, a multiplicative decomposition of the deformation model accounting for the active contraction of the areas of high actomyosin profile was developed. The numerical results shows a $55 \%$ closure of the wound, with a decreasing velocity until a stable diameter is reached, as seen in Figure 17.

In order to explain this divergences, several hypothesis are presented. The first one, and the simplest, is related with the modeling of the wound healing. Both the model itself or the tuning of the parameters involved in the coupled equations could not be the right choice or it could only be for a certain combination of parameters. We did an extensive analysis of the material parameters so we believe that the model could not reproduce all the biological phenomena in wound healing. For example, in the calcium diffusion equation, the matrix of diffusion may be nor isotropic nor constant, and its coefficients may vary with time, concentration or even strain rate.

In addition, the viscous effects during the wound expansion stage could be relevant in the initial mechanical contractions as well as the effect of the adhesion between the epithelial tissue and the extracellular matrix. Also, the hyperelastic model considered does not account for damage, which would modify the stress distribution, specially along the wound margin cells. Regarding the calcium wave during the transient state, no explanation of how the initial profile at $40-1500 \mathrm{~s}$ observed in Figure 3 is achieved was provided by literature or by our theoretical description. There exists a wave of calcium that goes outwards and comes back to the wound edge, resulting in the final purse-string at the wound edge. The actomyosin wave that advances, Figure 4, suggests that a given calcium concentration promotes the recruitment of actin filaments but also high or low concentrations promote the destruction of them as the original profile is retrieved 
once the actin wave passes by. This destruction or sharp decrease is not included in our model. We note though that experimental biological insights in the problem have not been able to solve these orchestrated mechanisms and, therefore, further experimental and theoretical studies will be required in the future.

In terms of the purse-ring contraction, two factors could explain the fact that closing velocity decreases as the wound diameter is smaller. In one hand, the kinetic model accounts for the overlap of actin filaments and it has been adopted in the proposed model as the main mechanical process toward wound closure. However, it has been proposed that additionally to the myosin motor action, there also exists a process of depolimerization of the filaments [33], that would result in a lower overlap and, as a consequence, an evolution of the active contraction independent to this parameter. In the other hand, the model implemented takes into account the elastic deformation in order to calculate the active stretch velocity, which as it gets nearer to the original equilibrium state reduces the active contraction update speed. Finally, no cell crawling contribution was taken into account in the numerical model, as well as other cell behaviours, which would explain the discrepancy at the final closing stage.

\section{Conclusion}

A mathematical and numerical model was developed, to account for the short and long term mechanism guiding the healing of epithelial wounds. To the best of the authors knowledge, this is the first model of its kind applied in this particular field in which the chemical and mechanical aspects of the problem were coupled. Hopefully it will encourage new developments in the subject of mechano-chemical sensing in wound healing. Firstly, the complex interplay of biochemical factors as result of the wound formation was considered. The biochemical processes that modify the intracellular actomyosin distribution and, in consequence, the contractility at cell level that was modeled. To do so a coupled system of non-linear Partial Differential Equations was considered and solved monolithically within a finite element framework. Later, the long term effects in the system were considered, i.e the purse-string mechanism. Within the finite element approach, the contractibility effect was included by adopting a classical Hill's assumption from the context of muscle contraction. In order to do this, a multiplicative and additive decomposition of the deformation gradient and strain energy density function were taken into account. The model considered the power done by the actomyosin element driving the wound closure. Although some authors have tried to model and simulate this problem, this is the first time that this mechanism is defined within a coupled non-linear finite element framework.

Although the actomyosin ring formation model was implemented successfully, the numerical results obtained do not fit perfectly the experimental data at intermediated transient stages in a satisfactory way. Although the intermediate stages of calcium concentration and actin recruitment are not exactly reproduced, the long term formation of the actin purse-string was successfully achieved. Regarding the active contraction of the actomyosin cable, contractions were achieved consequent partial closure of the wound gap. This behavior suggests that farther biological phenomena and signalling needs to be included in the model. Considerations like variable diffusivity parameters, with different coefficients in circumferential and radial directions, or adhesion between the tissue and the substrate, among other aspects, could be the key to solve the mentioned problems.

To conclude, it is believed that new light has been shed for a better understanding of wound healing by taking into account the interaction between active mechanical contraction and transport of actin and calcium.

\section{Acknowledgments}

The authors are very thankful to Yanlan Mao at the Laboraory of Molecular and Cell Biology of University College London for her helpful discussions. P.S. and J.J.M. have been financially supported by the Spanish Ministry of Economy and Competitiveness (MiNeCo) under grants K-00555 and DPI2016-74929-R, and by Generalitat de Catalunya under grants 2014-SGR-1471 and 2017-SGR-1278. 


\section{References}

[1] A. Jacinto, A. Martinez-Arias, P. Martin. Mechanisms of epithelial fusion and repair, Nature Cell Biology 3 (5), E117-E123, 2001.

[2] A. Ravasio, I. Cheddadi, T. Chen, T. Pereira, H. T. Ong, C. Bertocchi, A. Brugues, A. Jacinto, A. J. Kabla, Y. Toyama, X. Trepat, N. Gov, L. Neves de Almeida, B. Ladoux. Gap geometry dictates epithelial closure efficiency, Nature Communications 6:7683, 2015.

[3] E. Marinari, A. Mehonic, S. Curran, J. Gale, T. Duke, B. Baum. Live-cell delamination counterbalances epithelial growth to limit tissue overcrowding, Nature 484 (7395), 542-545, 2012.

[4] L. M. Crosby, C. M. Waters. Epithelial repair mechanisms in the lung, AJP: Lung Cellular and Molecular Physiology 298 (6), L715-L731, 2010.

[5] M. Antunes, T. Pereira, J. V. Cordeiro, L. Almeida, A. Jacinto. Coordinated waves of actomyosin flow and apical cell constriction immediately after wounding, The Journal of Cell Biology 202 (2), 365-379, 2013.

[6] S. Begnaud, T. Chen, D. Delacour, R. Mège, M. René and B. Ladoux. Mechanics of epithelial tissues during gap closure, Current Opinion in Cell Biology, 42:52-62, 2016.

[7] M. Stanisstreet, Calcium and wound healing in Xenopus early embryos. Journal of embryology and experimental morphology 67:195-205, 1982 .

[8] S. Xu, A.D. Chisholm. A G $\alpha \mathrm{q}-\mathrm{Ca} 2+$ signaling pathway promotes actin-mediated epidermal wound closure in C. Elegans, Current Biology, 21(23):1960-1967,2011.

[9] X. Soto, J. Li, R. Lea, E. Dubaissi, N. Papalopulu, and E. Amaya. Inositol kinase and its product accelerate wound healing by modulating calcium levels, Rho GTPases, and F-actin assembly, Proceeding of the National Academy of Sciences 110(27):11029-11034, 2013.

[10] M.V. Hunter, D.M. Lee, T.J.C. Harris, R. Fernandez-Gonzalez. Polarized E-cadherin endocytosis directs actomyosin remodeling during embryonic wound repair, Journal of Cell Biology, 210(5):801-816, 2015.

[11] E.K. Shannon, A. Stevens, W. Edrington, Y. Zhao, A.K. Jayasinghe, A. Page-McCaw, M.S. Hutson. Multiple mechanisms drive calcium signal dynamics around laser-induced epithelial wounds, Biophysical Journal, 113(7):1623-1635, 2017.

[12] T. J. Jentsch, C. A. Hubner, J. C. Fuhrmann. Ion channels: Function unravelled by dysfunction, Nature Cell Biology 6 (11), 1039-1047, 2004.

[13] B. Martinac. Mechanosensitive ion channels: molecules of mechanotransduction, Journal of Cell Science 117 (12), 2449 2460, 2004

[14] F. Sachs, Stretch-activated ion channels: what are they?, Physiology 25 (1), 50-56, 2010.

[15] P. Bagnerini, J. Lewis. Actin cables and epidermal movement in embryonic wound healing, Nature 360 (6400), 179-183, 1992.

[16] B. Garcia-Fernandez, I. Campos, J. Geiger, A. C. Santos, A. Jacinto. Epithelial resealing, The International Journal of Developmental Biology 53 (8-9-10), 1549-1556, 2009.

[17] G. P. S. Vedula. Mechanics of epithelial closure over non-adherent environments, Nature Communications 6:6111, 2015.

[18] J. Brock. Healing of incisional wounds in the embryonic chick wing bud: characterization of the actin purse-string and demonstration of a requirement for Rho activation, The Journal of Cell Biology 135 (4), 1097-1107, 1996.

[19] M. Tamada, T. D. Perez, W. J. Nelson, M. P. Sheetz. Two distinct modes of myosin assembly and dynamics during epithelial wound closure, The Journal of Cell Biology 176 (1), 27-33, 2007.

[20] W. Wood, A. Jacinto, R. Grose, S. Woolner, J. Gale, C. Wilson, P. Martin. Wound healing recapitulates morphogenesis in Drosophila embryos, Nature Cell Biology 4 (11), 907-912, 2002.

[21] S.K. Yoo, C.M. Freisinger, D.C. LeBert, A. Huttenlocher. Early redox, Src family kinase, and calcium signaling integrate wound responses and tissue regeneration in zebrafish, Journal Cell Biology, 199(2):225-234, 2012.

[22] A. Brugués, E. Anon, V. Conte, J. H. Veldhuis, M. Gupta, J. Colombelli, J. J. Muñoz, G. W. Brodland, B. Ladoux, X. Trepat. Forces driving epithelial wound healing, Nature Physics 10 (9), 683-690, 2014.

[23] J. H. Kim, X. Serra-Picamal, D. T. Tambe, E. H. Zhou, C. Y. Park, M. Sadati, J. Park, R. Krishnan, B. Gweon, E .Millet, J. P. Butler, X. Trepat, J. J. Fredberg. Propulsion and navigation within the advancing monolayer sheet, Nature Materials $12(9), 856-863,2013$.

[24] J. Long, M. Junkin, P. K. Wong, J. Hoying, P. Deymier. Calcium wave propagation in networks of endothelial cells: model-based theoretical and experimental study, PLoS Computational Biology 8 (12), e1002847, 2012.

[25] T. Hofer, A. Politi, R. Heinrich. Intercellular Ca2+ wave propagation through Gap-junctional Ca2+ diffusion: a theoretical study, Biophysical Journal 80 (1), 75-87, 2001.

[26] M. Goldberg, M. De Pitta, V. Volman, H. Berry, E. Ben-Jacob. Nonlinear Gap junctions enable long-distance propagation of pulsating calcium waves in astrocyte networks, PLoS Computational Biology 6 (8), e1000909, 2010.

[27] M. T. Abreu-Blanco, J. M. Verboon, R. Liu, J. J. Watts, S. M. Parkhurst. Drosophila embryos close epithelial wounds using a combination of cellular protrusions and an actomyosin purse string, Journal of Cell Science 125 (24), 5984-5997, 2012 .

[28] J. K. Klarlund. Dual modes of motility at the leading edge of migrating epithelial cell sheets, Proceedings of the National Academy of Sciences 109 (39), 15799-15804, 2012.

[29] E. Anon, X. Serra-Picamal, P. Hersen, N. C. Gauthier, M. P. Sheetz, X. Trepat, B. Ladoux. Cell crawling mediates collective cell migration to close undamaged epithelial gaps, Proceedings of the National Academy of Sciences 109 (27), 10891-10896, 2012.

[30] G. Fenteany, P. Janmey, T. P. Stossel. Signaling pathways and cell mechanics involved in wound closure by epithelial cell sheets, Current Biology 10 (14), 831-838, 2000. 
[31] J. Rosenblatt, M. C. Raff, L. P. Cramer. An epithelial cell destined for apoptosis signals its neighbors to extrude it by an actin- and myosin-dependent mechanism, Current Biology 11 (23), 1847-1857, 2001.

[32] W. M. Bement, C. A. Mandato, M. N. Kirsch. Wound-induced assembly and closure of an actomyosin purse string in Xenopus oocytes, Current Biology 9 (11), 579-587, 1999.

[33] A. C. Martin. Embryonic ring closure: actomyosin rings do the two-step, The Journal of Cell Biology 215 (3), 301-303, 2016.

[34] P.D. Dale, J.A. Sherratt, P.K. Maini. A mathematical model for collagen fibre formation during foetal and adult dermal wound healing, Proceedings of the Royal Sociaty of London B, 263, 653-660, 1996.

[35] C. Valero, E. Javierre, J. M. Garcia-Aznar, M. J. Gomez-Benito. Regulatory mechanism involving feedback between contraction and tissue formation guides wound healing progression, PLoS ONE 9 (3), e92774, 2014.

[36] L.G. Bowden, H.M. Byrne, P.K. Maini, D.E. Moulton, A morphoelastic model for dermal wound closure, Biomechanics and Modeling in Mechanobiology, 15, 663-681, 2016.

[37] A. Buganza Tepole. Computational systems mechanobiology of wound healing, Computer Methods in Applied Mechanics and Engineering (314), $46-70,2017$.

[38] L. Almeida, P. Bagnerini, A. Habbal, S. Noselli, F. Serman. A mathematical model for dorsal closure, Journal of Theoretical Biology, 268 (1), 105-119, 2011.

[39] L. Almeida, P. Bagnerini, A. Habbal. Modeling actin cable contraction, Computers \& Mathematics with Applications 64 (3), 310-321, 2012.

[40] T. Nagai, H. Honda. Computer simulation of wound closure in epithelial tissues: Cell basal lamina adhesion, Physical Review E 80 (6), 2009.

[41] P. Mosaffa, A. Rodríguez-Ferran, J. Muñoz. Hybrid cell-centred/vertex model for multicellular systems with equilibriumpreserving remodelling, International Journal For Numerical Methods in Biomedical Engineering, Accepted.

[42] I. Gonzalez-Valverde, J.M. Garcína-Aznar. Mechanical modeling of collective cell migration: An agent-based and continuum material approach, Computer Methods in Applied Mechanics and Engineering 337, 246 - $262,2018$.

[43] E. Kuhl, P. Steinmann. Theory and numerics of geometrically non-linear open system mechanics, International Journal for Numerical Methods in Engineering 58 (11), 1593-1615, 2003.

[44] E. K. Rodriguez, A. Hoger, A. D. McCulloch. Stress-dependent finite growth in soft elastic tissues, Journal of Biomechanics 27 (4), 455-467, 1994.

[45] E. H. Lee. Elastic-plastic deformation at finite strains, Journal of Applied Mechanics 36 (1),1-6, 1969.

[46] J. Donea, A. Huerta. Finite element methods for flow problems, Wiley, Hoboken, NJ, 2003.

[47] T. Toyofuku, M. Yabuki, K. Otsu, T. Kuzuya, M. Hori, M. Tada. Intercellular calcium signaling via gap junction in connexin-43-transfected cells, Journal of Biological Chemistry 273(3):1519-1528, 1998.

[48] N. R. Jorgensen, S.T. Geist, R. Civitelli, T.H. Steinberg. ATP- and gap junction-dependent intercellular calcium signaling in osteoblastic cells. The Journal of Cell Biology 139(2):497-506, 1997.

[49] P. Germain. The method of virtual power in continuum mechanics. Part 2: Microstructure, SIAM Journal on Applied Mathematics 25 (3), 556-575, 1973.

[50] G. A. Holzapfel. Nonlinear Solid Mechanics: A Continuum Approach for Engineering, John Wiley \& Sons, 2000.

[51] J. Grasa, B. Hernandez-Gascon. A 3d elecro-mechanical continuum model for simulating skeletal muscle contraction, Journal of Theoretical Biology 335:108-118, 2013.

[52] P. Sáez. On the theories and numerics of continuum models for adaptation processes in biological tissues, Archives of Computational Methods in Engineering 23 (2), 301-322, 2016.

[53] J. Wong, S. Goktepe. Computational modeling of chemo-electro-mechanical coupling: A novel implicit monolithic finite element approach, International Journal For Numerical Methods in Biomedical Engineering (29):1104-1133, 2014.

[54] H. Sackin. Stretch-activated ion channels, Kidney International 48:1134-1147, 1995.

[55] C. E. Morris. Mechanosensitive ion channels, The Journal of Membrane Biology 113 (2), 93-107, 1990.

[56] F. Guharay, F. Sachs. Stretch-activated single ion channel currents in tissue-cultured embryonic chick skeletal muscle, The Journal of Physiology 352, 685-701, 1984.

[57] B. Calvo, A. Ramírez, A. Alonso, J. Grasa, F. Soteras, R. Osta, M.J. Muñoz. Passive nonlinear elastic behaviour of skeletal muscle: Experimental results and model formulation, Journal of Biomechanics 43 (2), 318-325, 2010.

[58] S. Goktepe, A. Menzel, E. Kuhl. The generalized Hill model: A kinematic approach towards active muscle contraction, Journal of the Mechanics and Physics of Solids 72:20-39, 2014.

[59] A.G. Clark, A.L. Miller, E. Vaughan, H.Y.E. Yu, R. Penkert, W.M. Bement. Integration of single and multicellular wound responses. Current Biology 16(19): 1389-1395, 2009.

[60] K.J. Sonnemann, W.M. Bement. Wound Repair: Toward understanding and integration of single-cell and multicellular wound responses, Annual Review Cell Developmental Biology 27(1):237-263, 2011.

[61] W. Wood, Wound healing Calcium flashes illuminate early events. Current Biology 22(1):14-16, 2012. ti

[62] H. Benink, W.M. Bement. Concentric zones of active RhoA and Cdc42 around single cell wounds, Journal of Cell Biology 168(3):429-439, 2005. 
Appendix A. Actomyosin ring formation: parametric study 

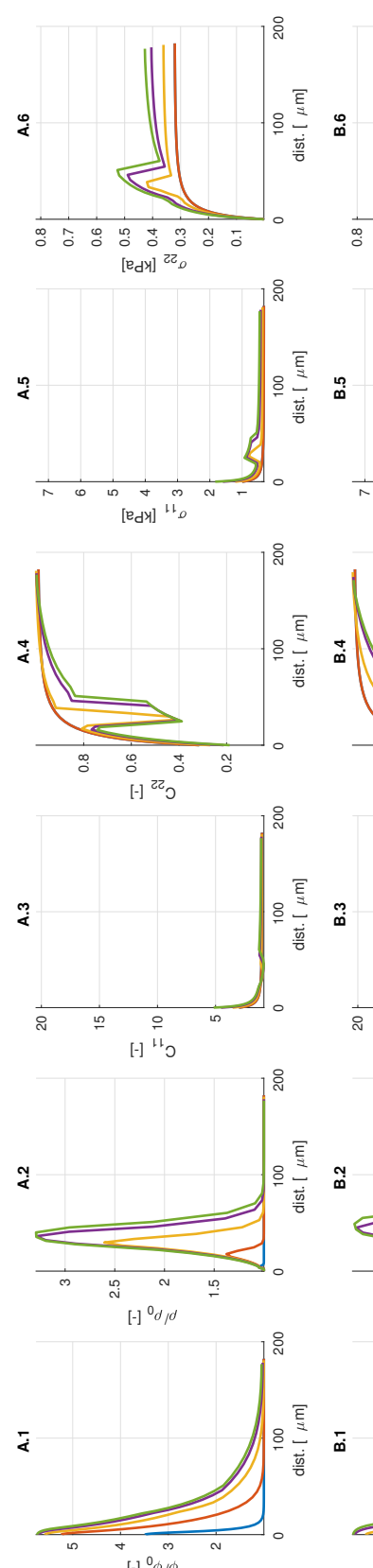

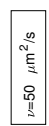
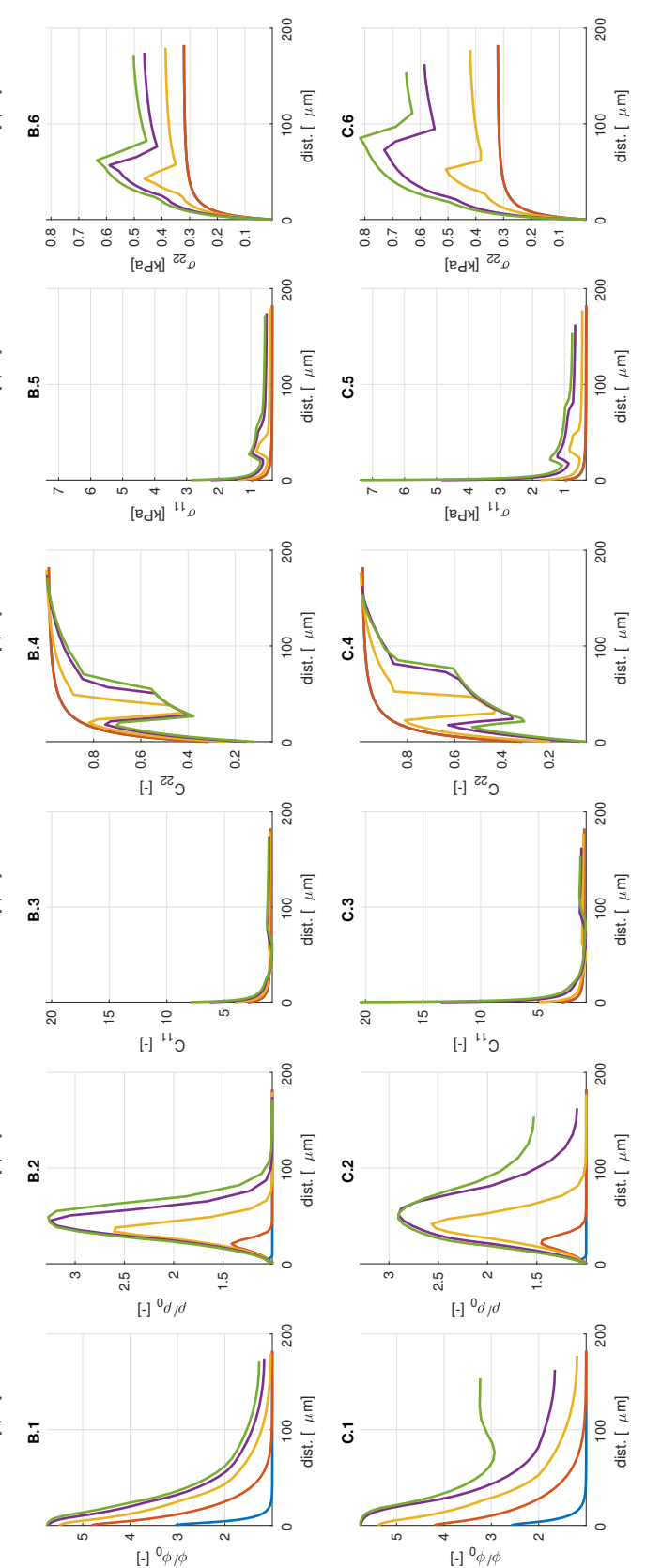

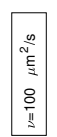
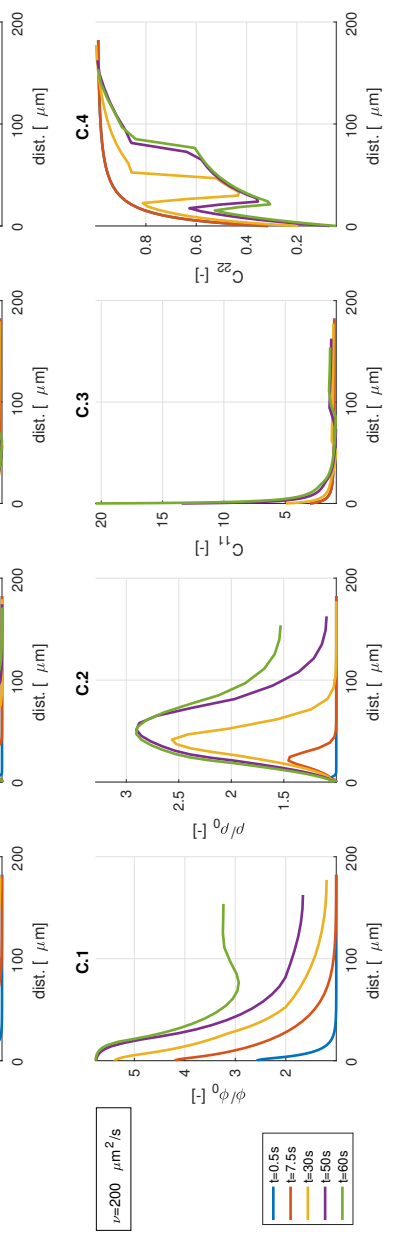

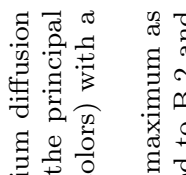

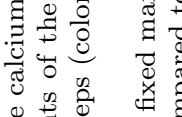

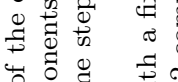
영

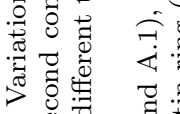
总: 可芯芯官

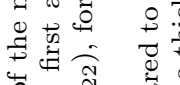

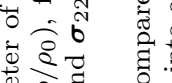
बे 可过

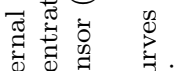
ब्व

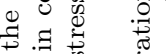
$\circ$ 范

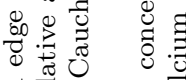

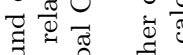
당

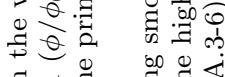

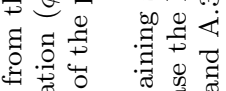

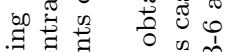

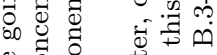

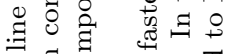
๙

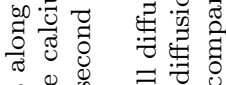

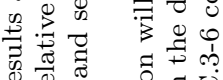
ซ్రై

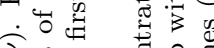
Iิ ชี ชูำ

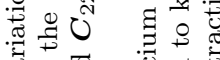

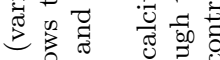

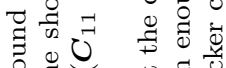
管

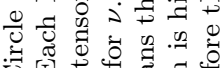
U되. $\ddot{0}$ 人

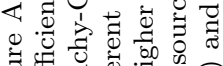

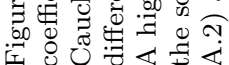




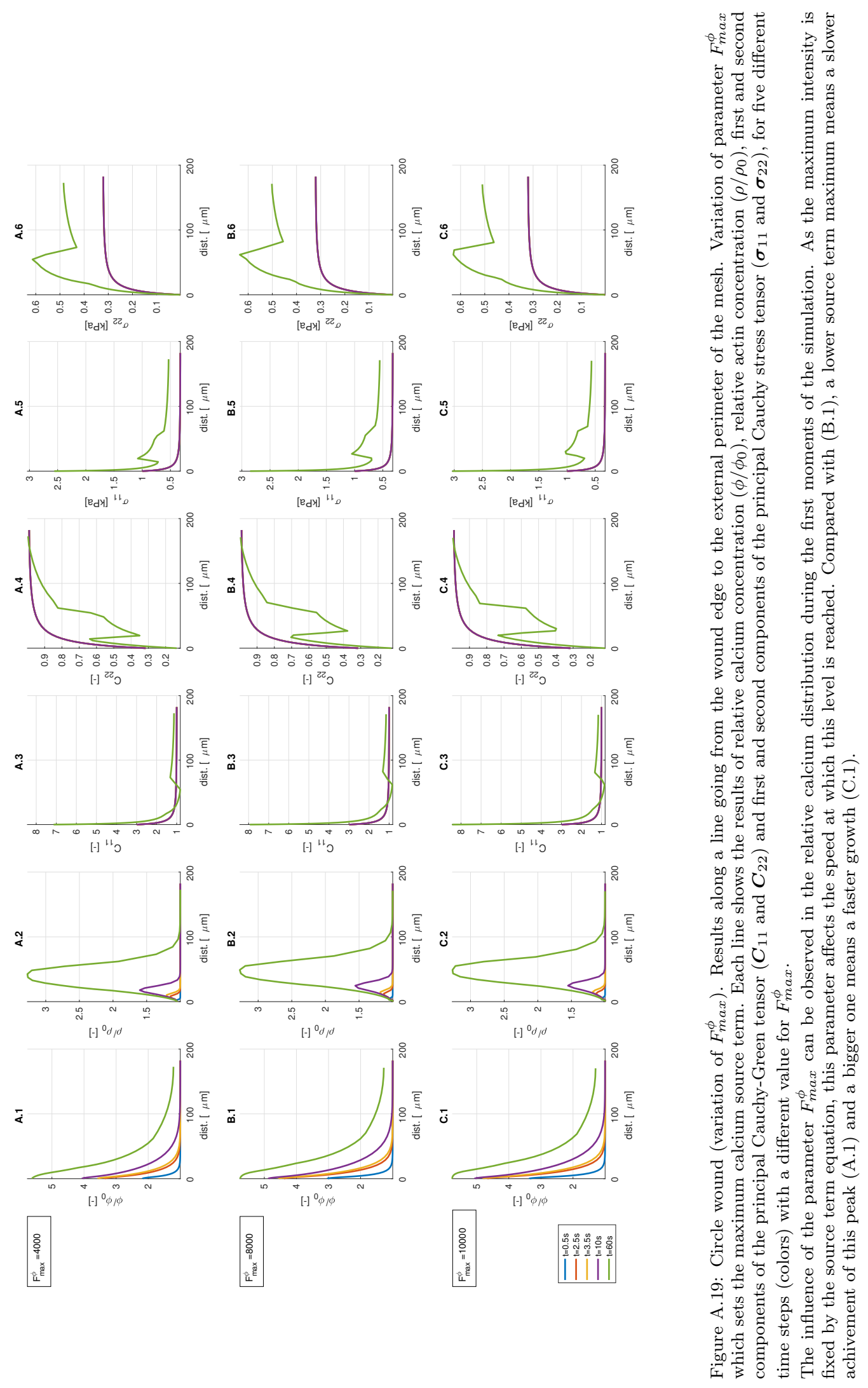



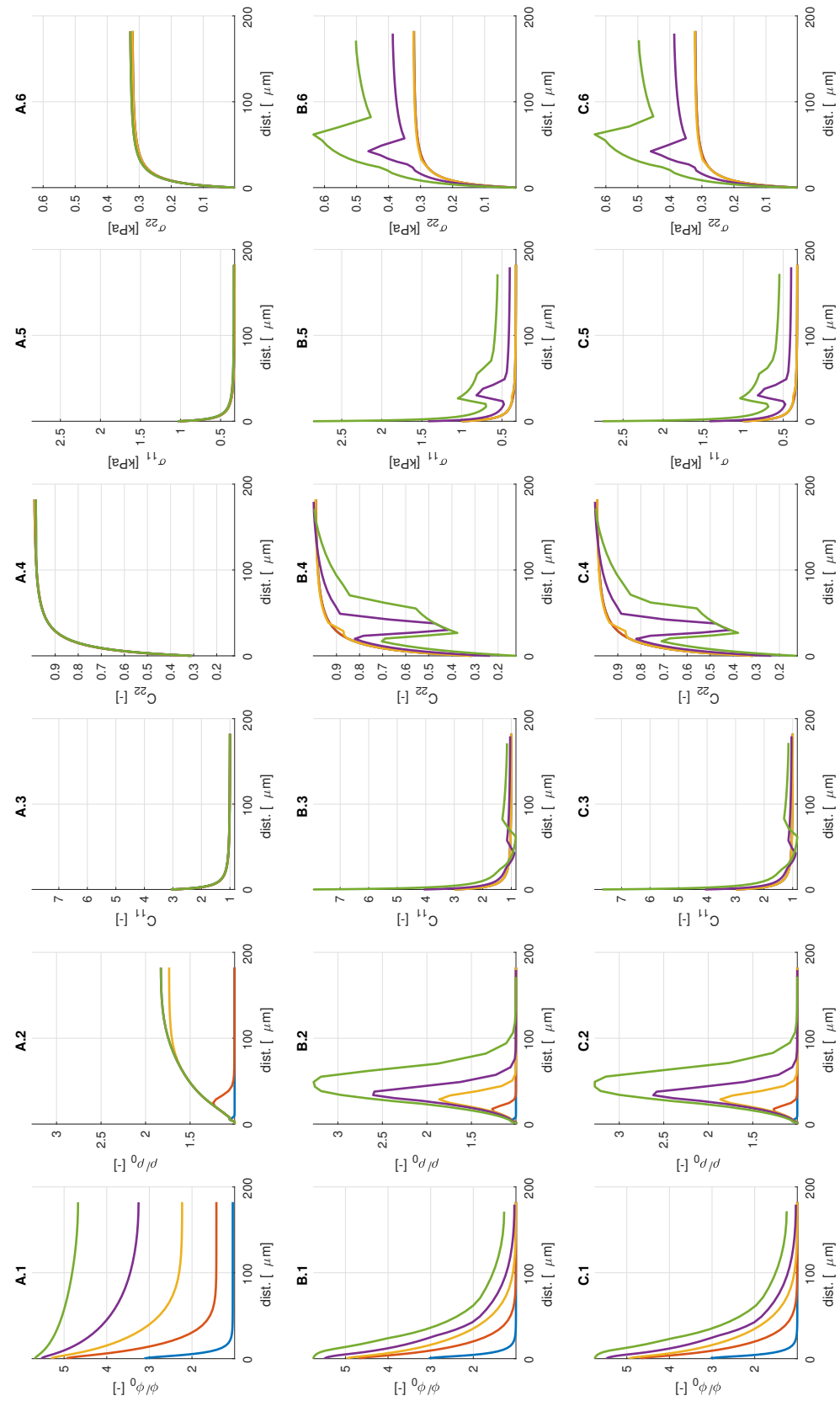

음
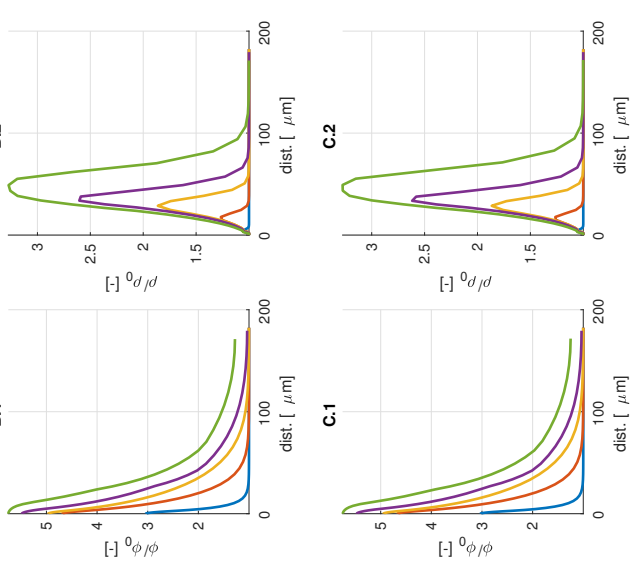

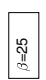

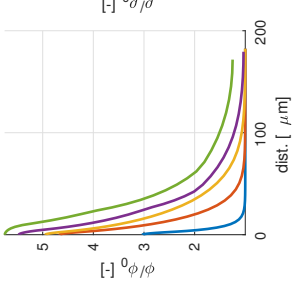

1
In
In

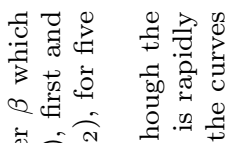

昰高

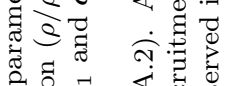

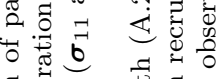

范荡

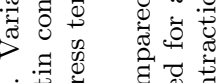

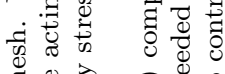

: $\bigcirc$

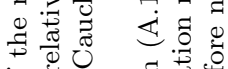

岁的政

Q

ठี

牙

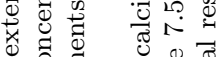

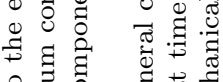

웅 矛

$\therefore$ ฮె

品

穿若

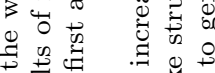

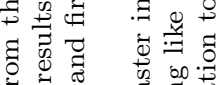

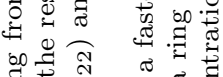

贻

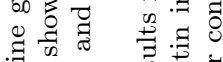

虽

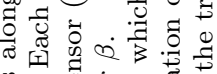

青守

ซ

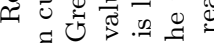

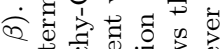

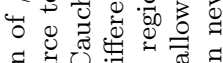

.

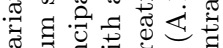

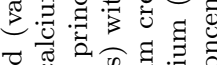

政

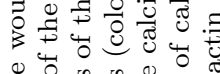

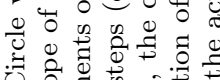

잉

คे

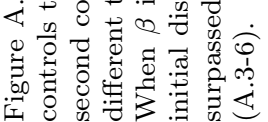



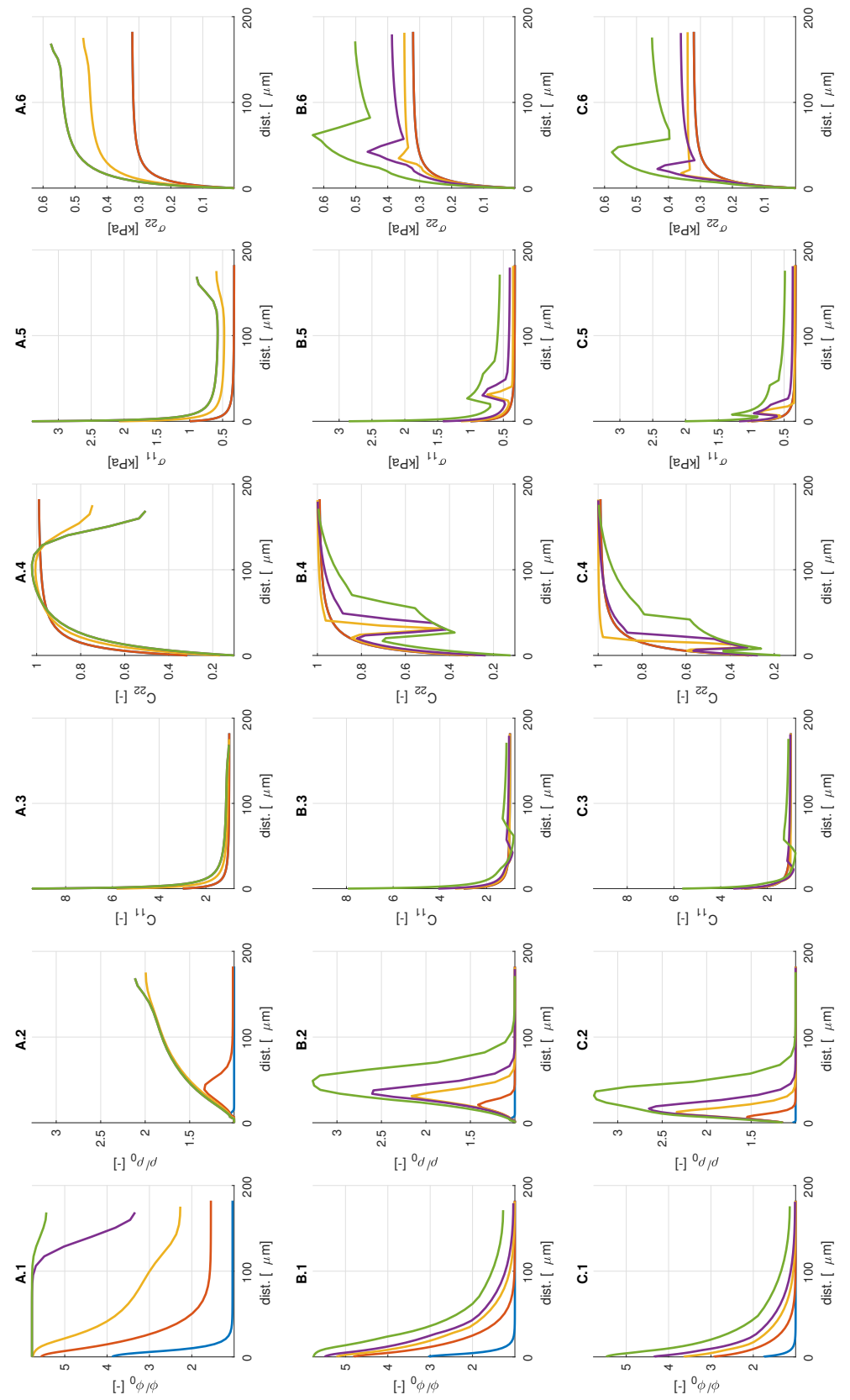

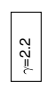
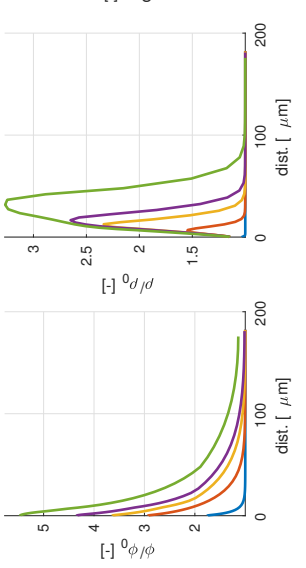

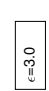

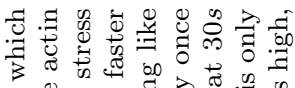

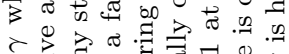

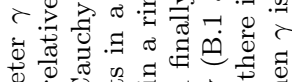

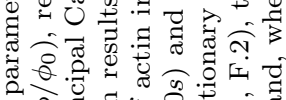

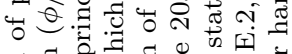

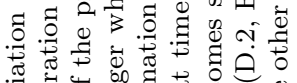

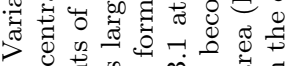

d

जै 承 Ð 厄ु

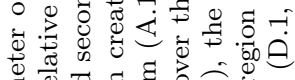

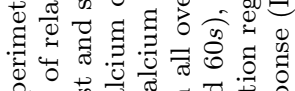

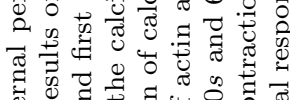
过

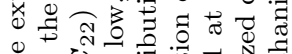

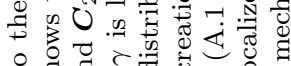
$\therefore \frac{8}{\omega}$ ส 品

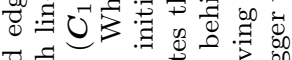

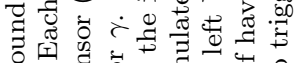

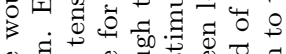

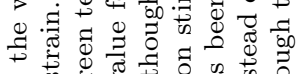

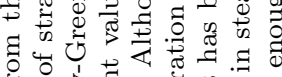

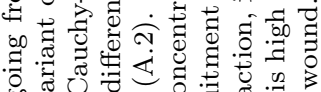

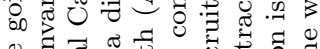
.

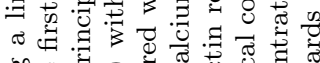

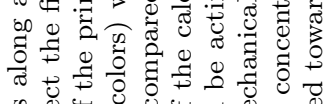

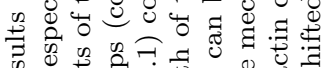

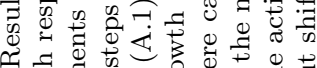

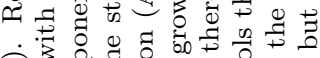
广ิ

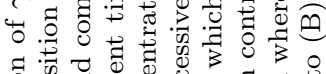

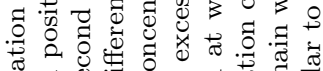

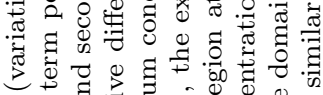

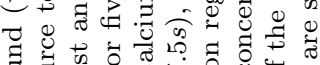

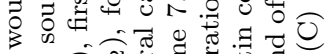

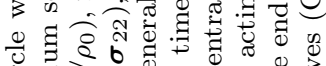

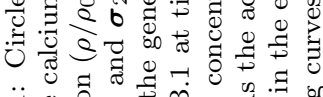

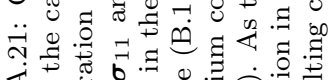

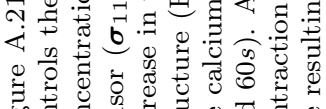



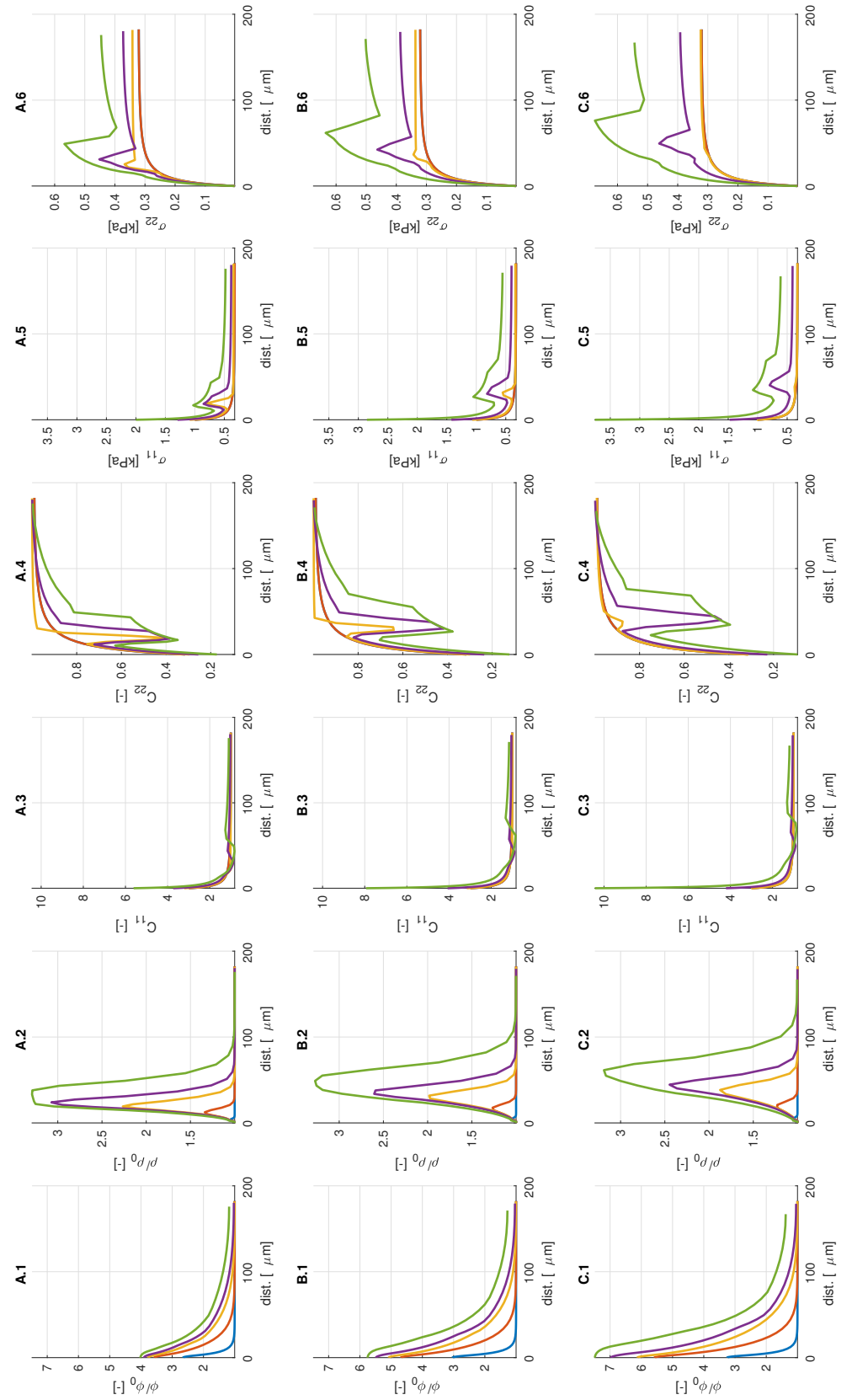

$\pi$
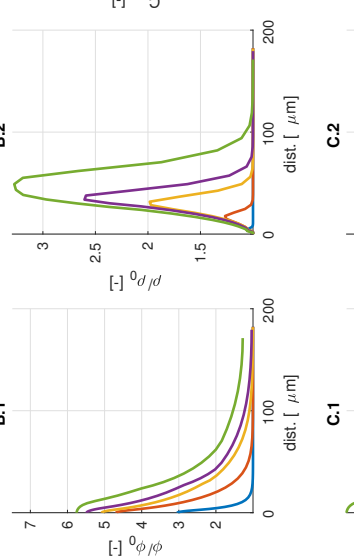

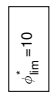
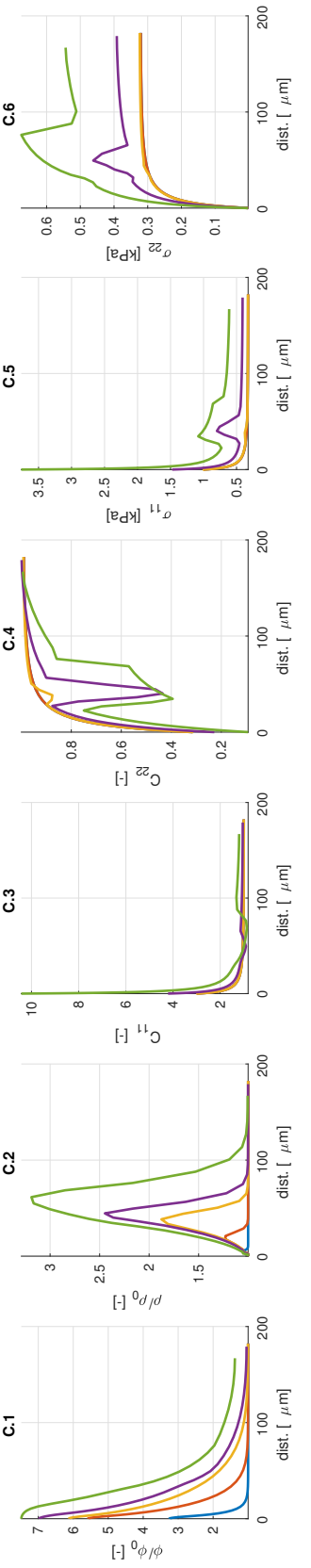

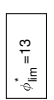

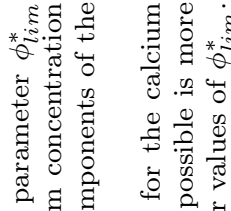

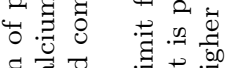

흄 च

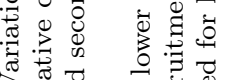

ᄀ芯范

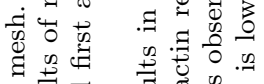

ब

证

पूत

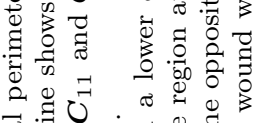

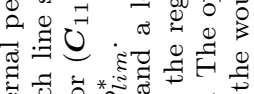

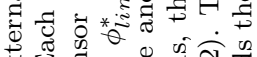
ชิ

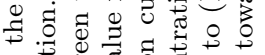

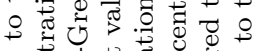

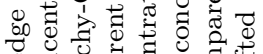
ه

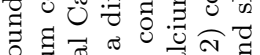
응.

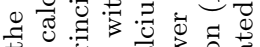

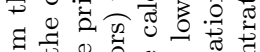

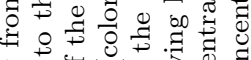
.

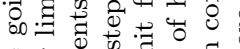

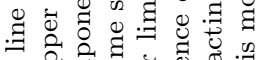
๘ $\exists$ :

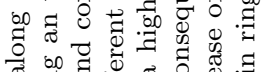
ส 殸施

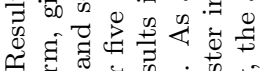

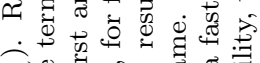
है붕 *

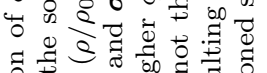

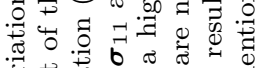

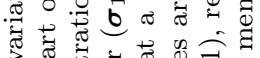

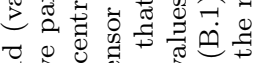

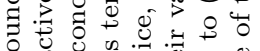

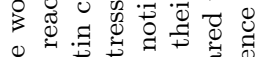

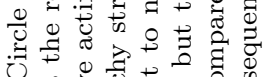

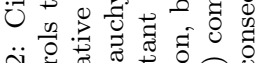
สี่

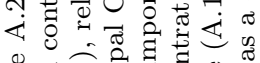

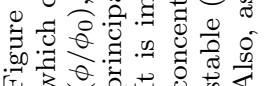




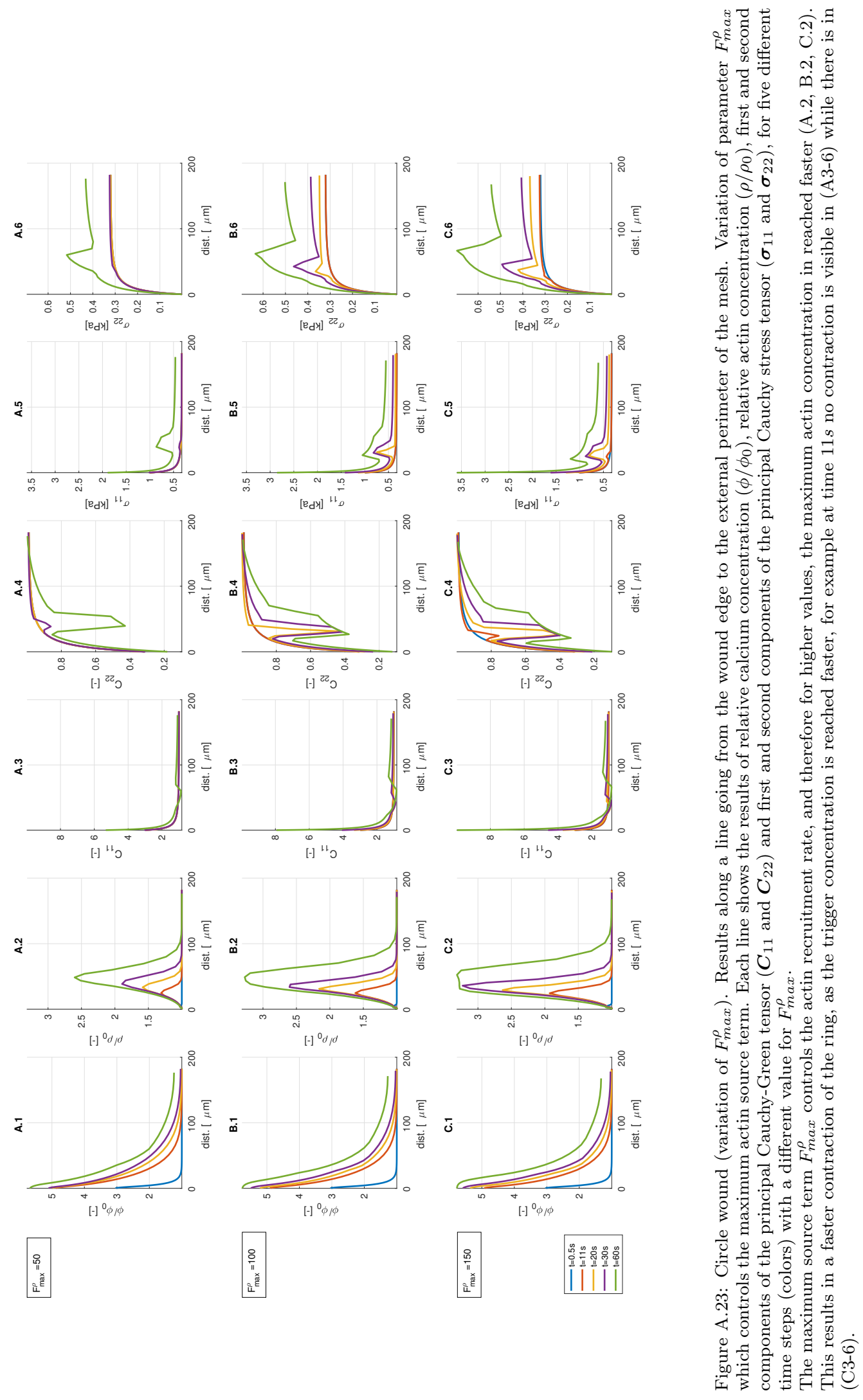



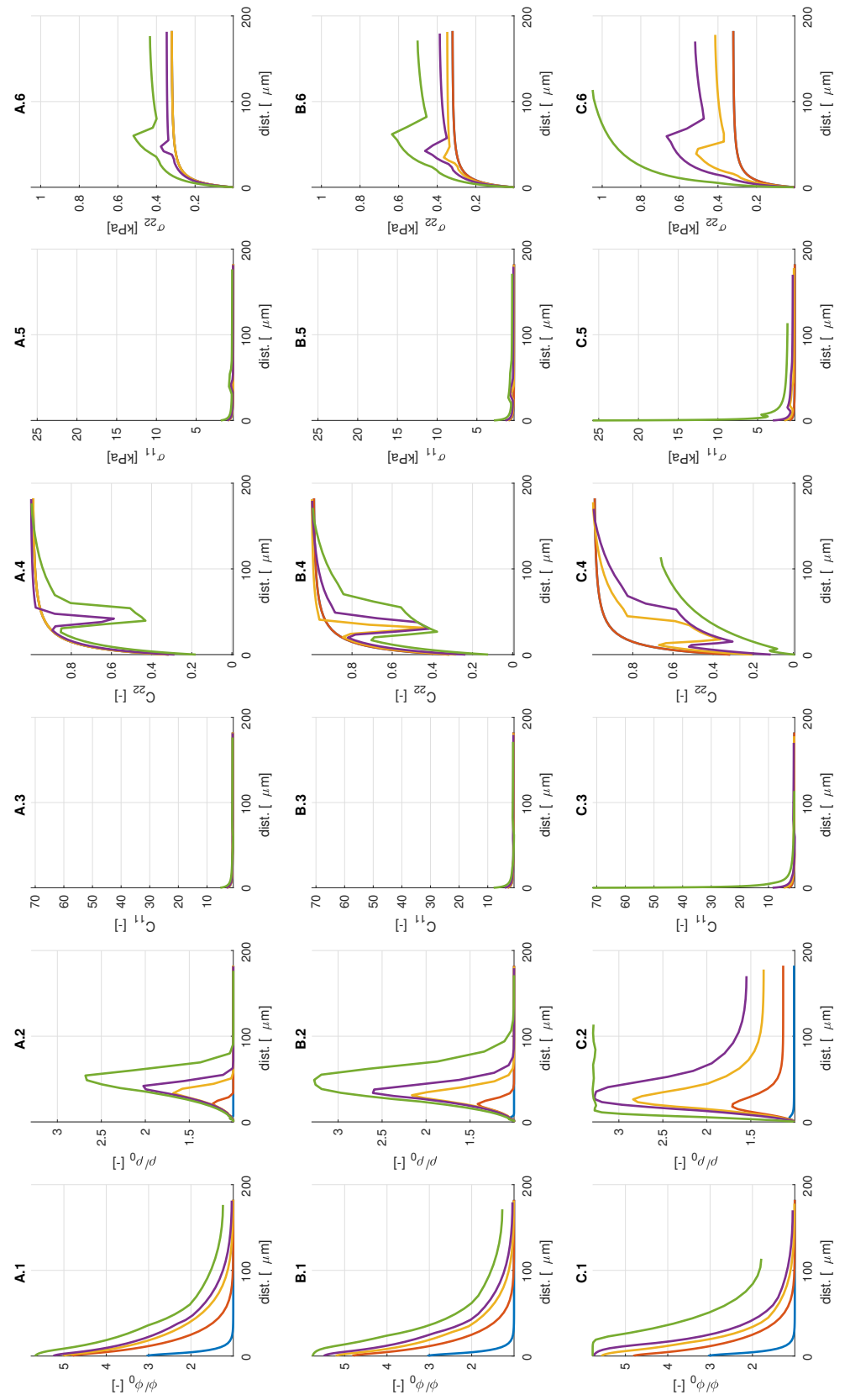

\begin{tabular}{|c|}
\hline \\
\hline 0 \\
II \\
\hline 1
\end{tabular}

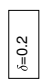
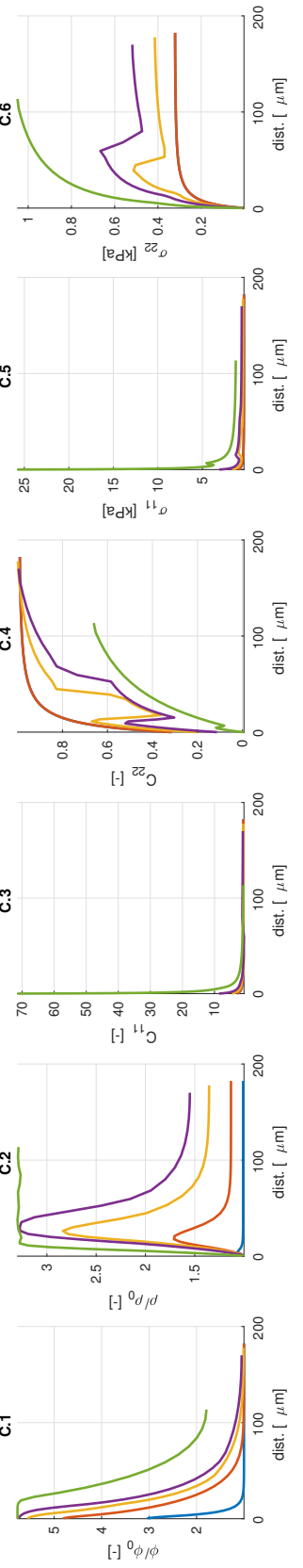

\begin{tabular}{|l|}
0 \\
0 \\
0 \\
01 \\
0
\end{tabular}

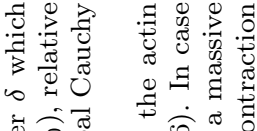

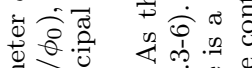

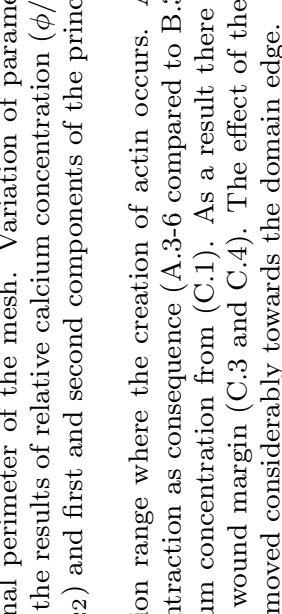

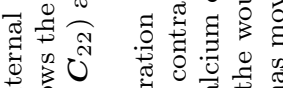
ख ‡.

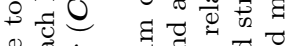

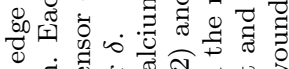
ช.

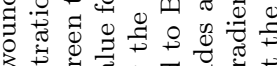

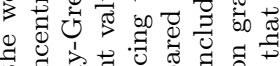

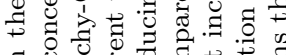

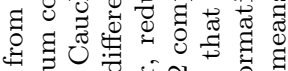

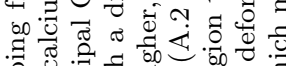
\&o

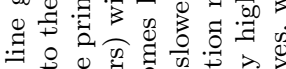

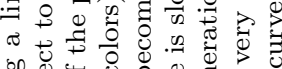

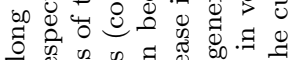
군

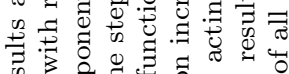

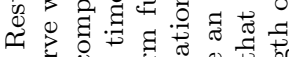
कि क 范出

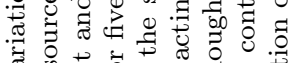

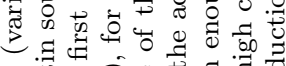

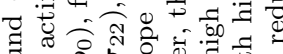
o ษ

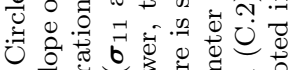

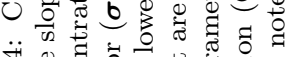

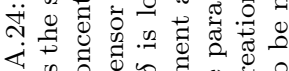

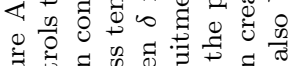

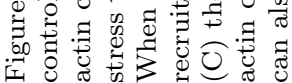



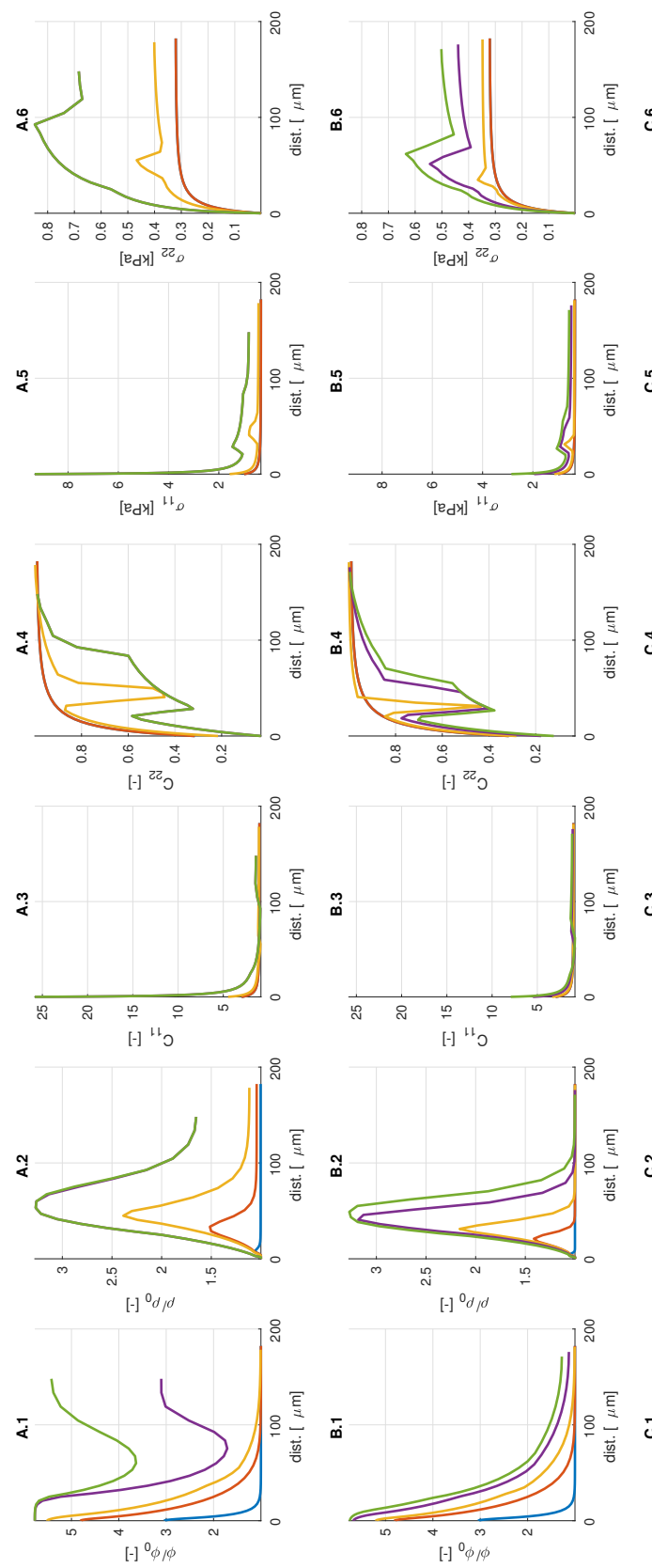

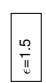
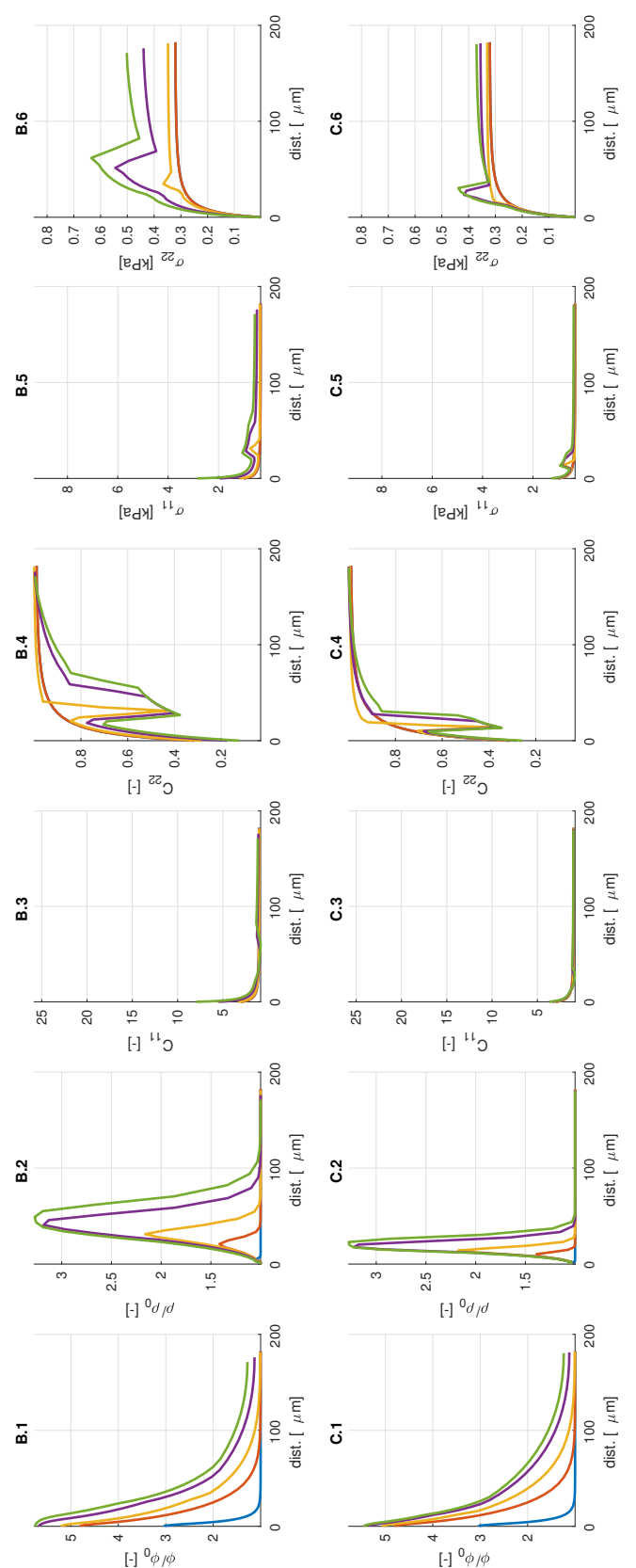

\begin{tabular}{|l|}
\hline \multirow{2}{*}{} \\
\\
\hline
\end{tabular}

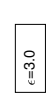

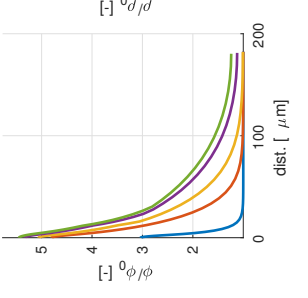

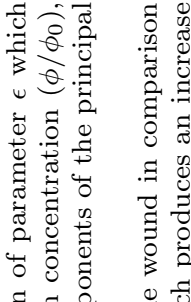

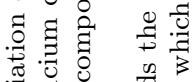

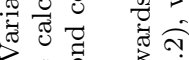

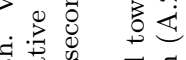

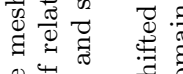

क्षे क्ष

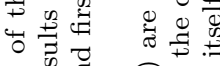

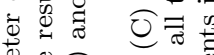

घั

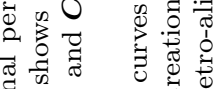

7 둥

可

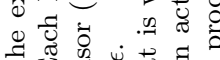

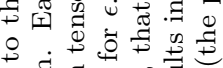

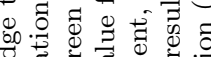

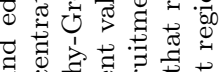

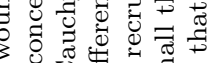
० 0 : ‡

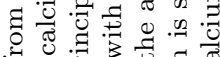

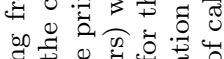

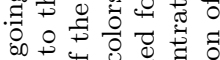
: कo

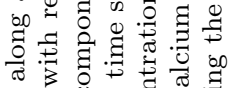

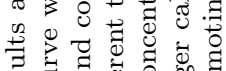

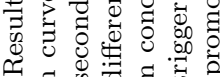

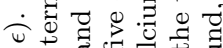

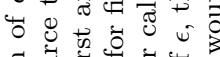

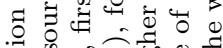
둥

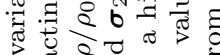

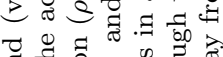

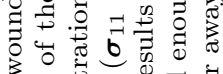
०

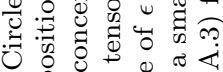

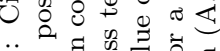

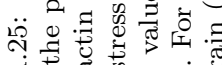

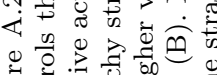

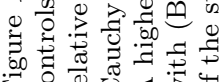




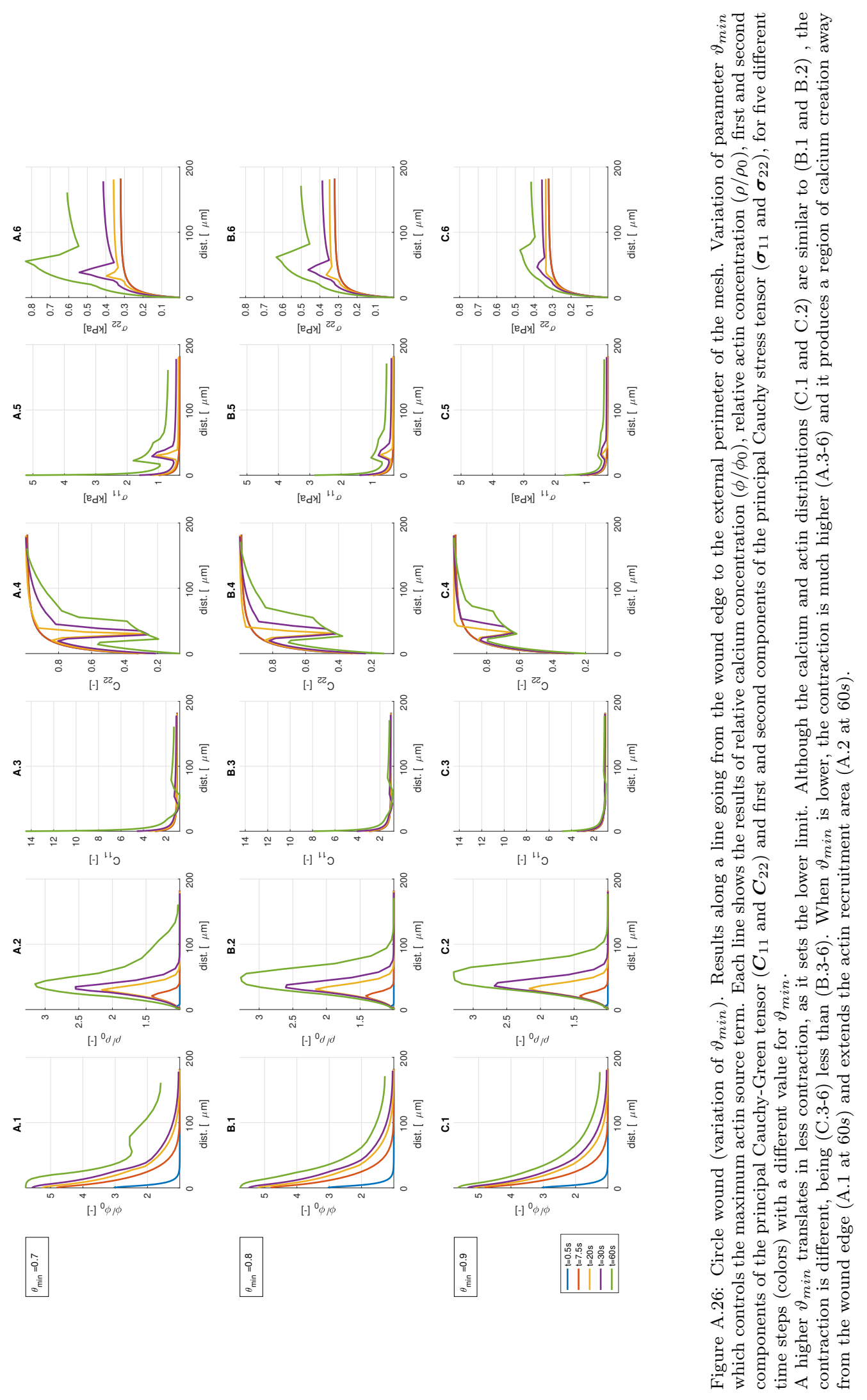




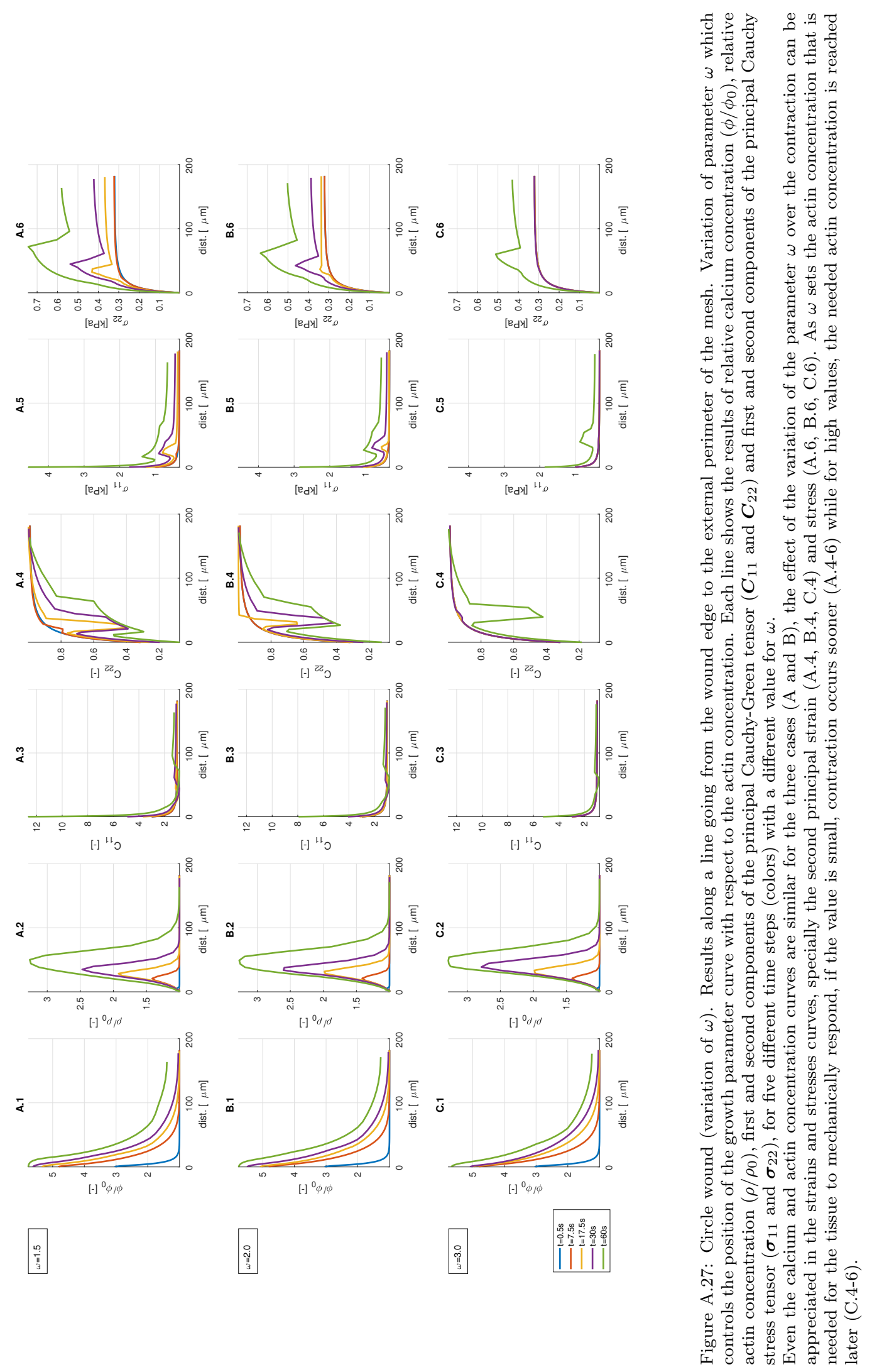




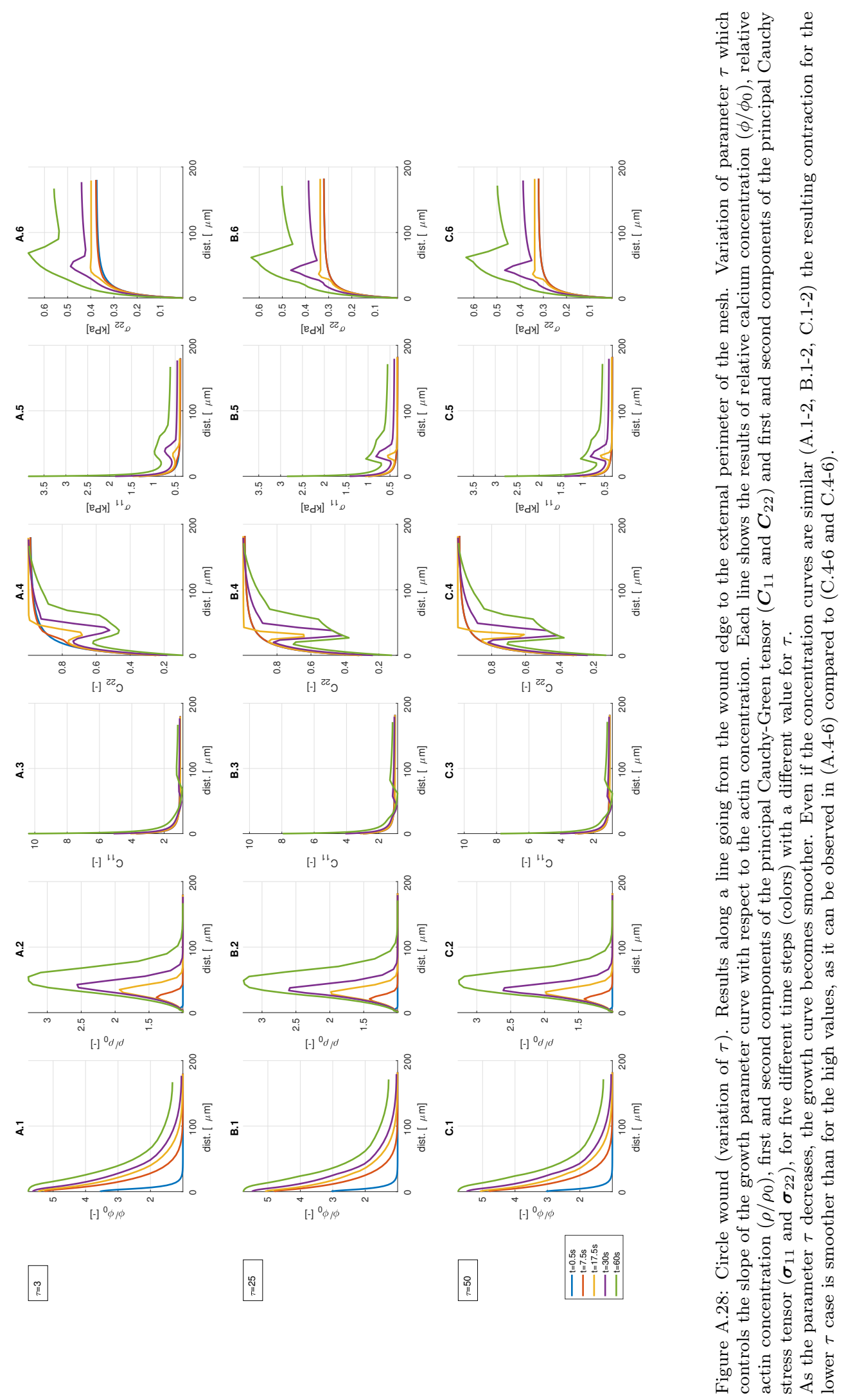

